\title{
An Upper Limit on the $B_{s}^{0} \rightarrow D_{s}^{-} \pi^{+}$Branching Ratio
}

\author{
A Dissertation \\ Presented to \\ The Faculty of the Graduate School of Arts and Sciences \\ Brandeis University \\ Physics Department \\ Dr. Craig Blocker, Advisor \\ In Partial Fulfillment \\ of the Requirements for the Degree \\ Doctor of Philosophy \\ by \\ Michael C. Kirk
}

February, 2003 
UMI Number: 3073889

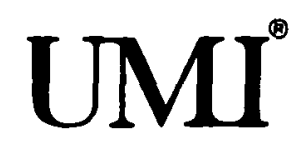

UMI Microform 3073889

Copyright 2003 by ProQuest Information and Learning Company.

All rights reserved. This microform edition is protected against unauthorized copying under Title 17, United States Code.

ProQuest Information and Learning Company

300 North Zeeb Road

P.O. Box 1346

Ann Arbor, MI 48106-1346 
This dissertation, directed and approved by Michael C. Kirk's Committee, has been accepted and approved by the Graduate Faculty of Brandeis University in partial fulfillment of the requirements for the degree of

\section{DOCTOR OF PHILOSOPHY}

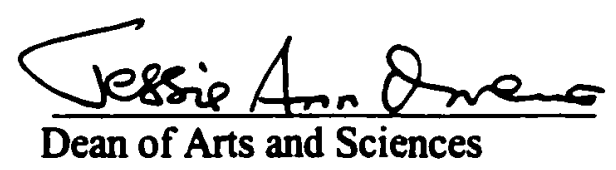

Dissertation Committee:

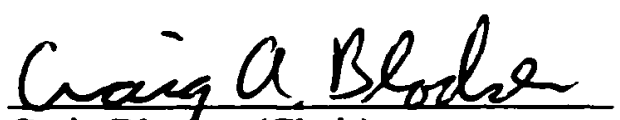

\section{Craig Blocker (Chair)}

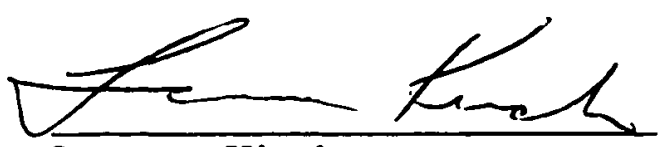

Lawrence Kirsch

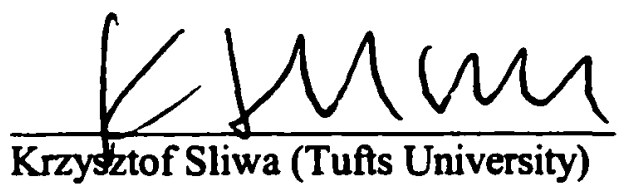


Copyright by Michael C. Kirk 


\section{ACKNOWLEDGMENTS}

I would like to thank my advisor Professor Craig Blocker for the influential role he played in my graduate education. His keen insight for solving problems, research prowess and approachable manner proved vital for the completion of my analysis. The way I now approach problems (especially statistical) and think about physics is a reflection of his efforts. I am very thankful for the chance to have worked with him.

I would also like to thank Professor Larry Kirsch for the opportunity to work on many interesting hardware and software related projects. I am grateful for the opportunity to learn from his expertise.

I would also like to recognize Kevin Pitts, Michael Schmidt, and Marjorie Shapiro for leading the Run I $b$ physics meetings. These provided a great opportunity to converse with other $b$ physics students and receive crucial feedback on the progress of my analysis. I am also very thankful for Jonathan Lewis, Barry Wicklund, Joe Kroll and other members of the $b$ physics groups for their insightful comments.

I am thankful to have had the opportunity to work with Fred Lewis. His electronics acumen gave me more time to work on this analysis and his basketball league gave me something to do when I needed a break.

I am deeply indebted to fellow Brandeis graduate student and officemate Hongquan Niu for his programming expertise and the many interesting political and spiritual debates. His friendship made daily life in the trailers much more interesting.

I would also like to thank fellow CDF graduate students, Tony Affolder and Masa Tanaka, 
for their assistance at various stages of my analysis.

Finally, and most importantly, I would like to recognize my family, without whom I would never have completed this work. I am very thankful to my parents for their continued love and support. From an early age they have given me the opportunity and motivation to pursue my goals. I cannot be more grateful for my lovely wife Shannon. Her patience and understanding during these final years has made this thesis possible. All accomplishments I've been blessed to have, I owe to them. Thank you. 


\title{
An Upper Limit on the $B_{s}^{0} \rightarrow D_{s}^{-} \pi^{+}$Branching Ratio
}

A dissertation presented to the Faculty of the Graduate School of Arts and Sciences of Brandeis University, Waltham, Massachusetts by Mlichael C. Kirk.

\begin{abstract}
This thesis reports the results of a study of $B \rightarrow D \pi$ decays. The analysis is performed on $110 \mathrm{pb}^{-1}$ of data collected by CDF at the Fermi National Accelerator Laboratory between 1992 and 1995. B mesons are reconstructed in the Run I inclusive lepton dataset in the opposite hemisphere from the lepton. $B_{d}$ and $B_{u}$ mesons are reconstructed via the $B \rightarrow D \pi$ channel for the first time in a hadron collider environment. The reconstructed $B_{u}$ and $B_{d}$ mesons serve as a reference signal for the $B_{s}$ search. Six $B_{s} \rightarrow D_{s} \pi$ decay modes are studied and an upper limit is placed on the $B_{s} \rightarrow D_{s} \pi$ branching ratio:
\end{abstract}

$$
\begin{gathered}
B R\left(B_{s} \rightarrow D_{s} \pi\right)<9.9 \% \text { at } 90 \% \text { C.L. } \\
B R\left(B_{s} \rightarrow D_{s} \pi\right)<15.9 \% \text { at } 95 \% \text { C.L. } \\
\text { and } \\
f\left(b \rightarrow B_{s}\right) \times B R\left(B_{s} \rightarrow D_{s} \pi\right)<1.6 \% \text { at } 90 \% \text { C.L. } \\
f\left(b \rightarrow B_{s}\right) \times B R\left(B_{s} \rightarrow D_{s} \pi\right)<2.6 \% \text { at } 95 \% \text { C.L. }
\end{gathered}
$$




\section{Contents}

I Theory 1

1 Introduction $\quad 1$

2 Standard Model $\quad 3$

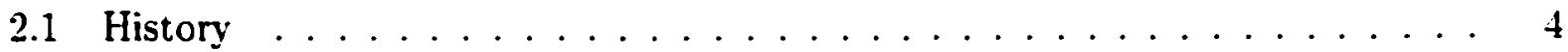

2.2 The Standard Model . . . . . . . . . . . . . . . . . .

2.2.1 Quantum Electrodynamics ................. 8

2.2.2 Quantum Chromodynamics .................. 8

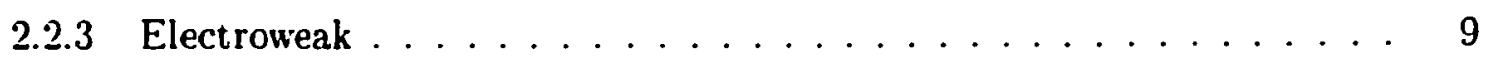

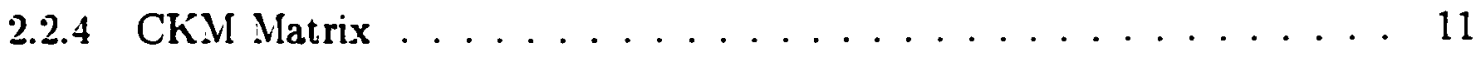

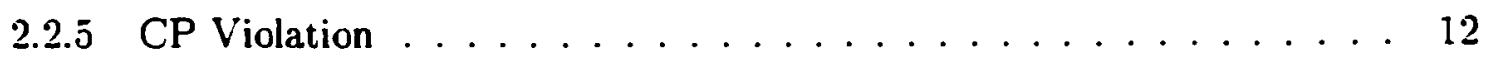

$3 \quad b \bar{b}$ Production $\quad 13$

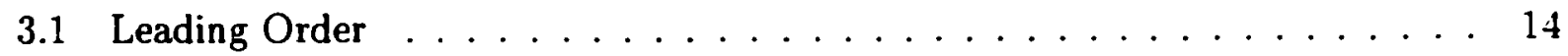

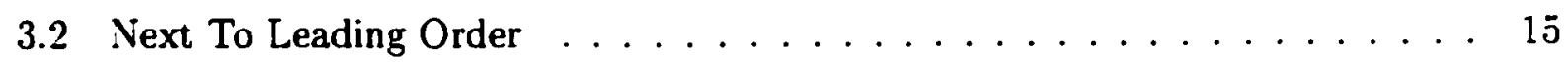

$3.3 \quad B$ Fragmentation . . . . . . . . . . . . . . . . 16

$\begin{array}{llr}4 & B \text { Mesons } & 18\end{array}$

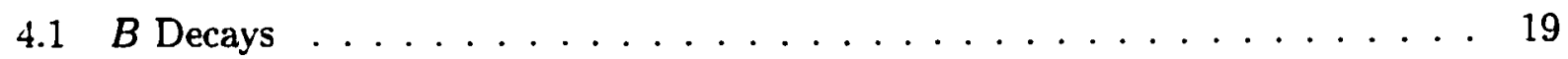

vii 
4.1 .1 Spectator Model . . . . . . . . . . . . . 20

4.1 .2 Branching Ratios . . . . . . . . . . . 21

$4.2 \quad B_{s}$ Mesons . . . . . . . . . . . . . . . . . 22

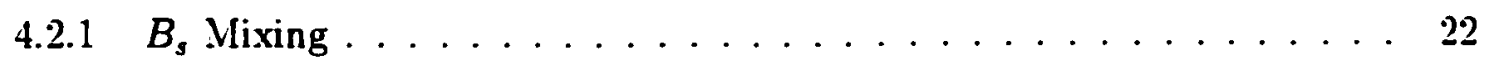

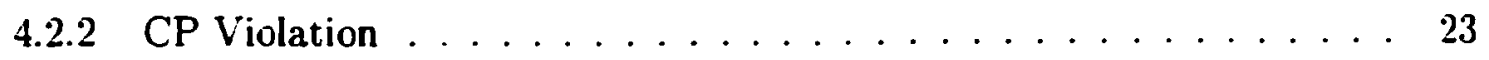

4.2 .3 Future Prospects $\ldots \ldots \ldots \ldots \ldots \ldots \ldots \ldots \ldots$

II Experimental Apparatus $\quad 24$

5 Accelerator $\quad 24$

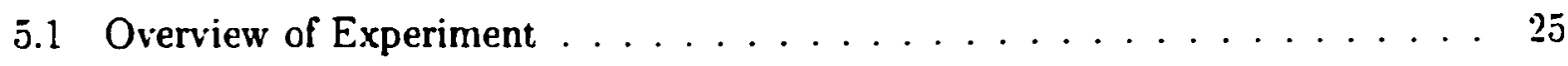

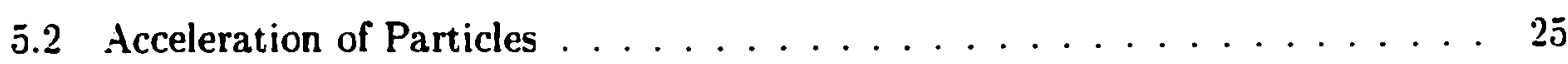

5.3 Particle Collisions $\ldots \ldots \ldots \ldots \ldots \ldots \ldots \ldots$

6 CDF Detector 29

6.1 Coordinate System . . . . . . . . . . . . . . . 29

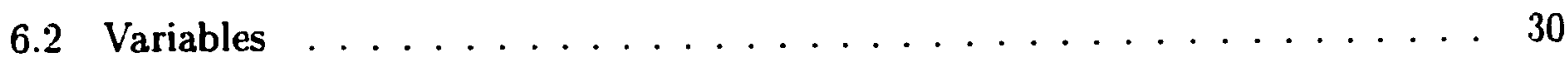

6.3 Detector Components $\ldots \ldots \ldots \ldots \ldots \ldots \ldots \ldots$

6.3.1 Silicon Vertex Detector $\ldots \ldots \ldots \ldots \ldots . \ldots \ldots$

6.3.2 Vertex Time Projection Chamber . . . . . . . . . 3 jృ

6.3.3 Central Tracking Chamber $\ldots \ldots \ldots \ldots \ldots \ldots \ldots$

viii 
6.3.4 Central Electromagnetic Calorimeter . . . . . . . . . . 37

6.3.5 Central Strip Chamber . . . . . . . . . . . . 38

6.3.6 Central Hadronic Calorimeter . . . . . . . . . . . . . 38

6.3.7 Muon Detectors $\ldots \ldots \ldots \ldots \ldots \ldots$

6.4 Trigger . . . . . . . . . . . . . . . . . . 39

III Analysis 43

7 Goal $\quad 43$

7.1 Relative Weasurement . . . . . . . . . . . . . . . 44

7.2 Reference Decay Modes . . . . . . . . . . . . . . . . 44

8 DataSet 45

8.1 Inclusive Lepton Dataset . . . . . . . . . . . . . . . 45

8.1.1 Electron Dataset . . . . . . . . . . . . . . . . . 47

8.1 .2 Conversion Electrons . . . . . . . . . . . . . . . . 48

8.1 .3 Muon Dataset $\ldots \ldots \ldots \ldots \ldots \ldots \ldots$

8.2 Monte Carlo . . . . . . . . . . . . . . . . . . . . . . 49

9 B Meson Reconstruction $\quad 50$

9.1 Decay Topology . . . . . . . . . . . . . . . . 51

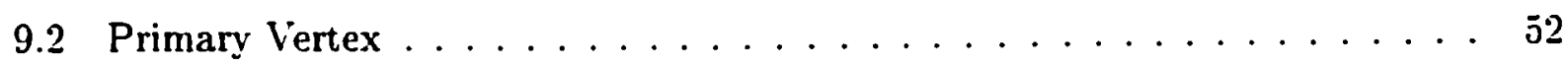




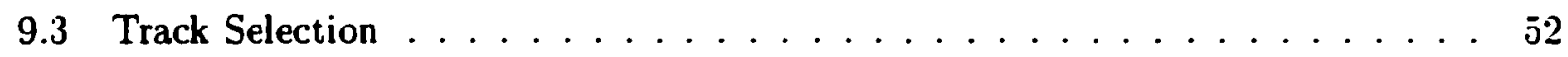

9.4 Reconstruction . . . . . . . . . . . . . . . 53

9.5 Summary of Reconstruction Cuts $\ldots \ldots \ldots \ldots \ldots \ldots \ldots \ldots$

10 Signal Optimization $\quad 55$

10.1 Sources of Background $\ldots \ldots \ldots \ldots \ldots \ldots \ldots \ldots \ldots \ldots \ldots$

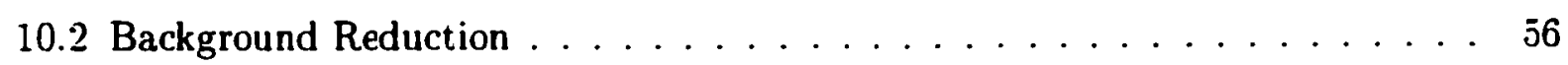

10.2.1 Transverse Momentum . . . . . . . . . . . . . 57

10.2.2 Proper Decay Time $\ldots \ldots \ldots \ldots \ldots \ldots \ldots$

10.2 .3 Isolation $\ldots \ldots \ldots \ldots \ldots \ldots \ldots \ldots \ldots \ldots \ldots$

10.3 Higher Order $B$ Decays $\ldots \ldots \ldots \ldots \ldots \ldots \ldots$

10.4 Optimization Procedure $\ldots \ldots \ldots \ldots \ldots \ldots \ldots$

10.4.1 $B^{+} \rightarrow \overline{D^{0}} \pi^{+}, \overline{D^{0}} \rightarrow K^{+} \pi^{-} \ldots \ldots \ldots \ldots \ldots \ldots \ldots$

$10.4 .2 B^{0} \rightarrow D^{-} \pi^{+}, D^{-} \rightarrow K^{+} \pi^{-} \pi^{-} \ldots \ldots \ldots \ldots \ldots \ldots \ldots \ldots \ldots \ldots$

10.4.3 $B^{+}$and $B^{0}$ Combined Result $\ldots \ldots \ldots \ldots \ldots \ldots$

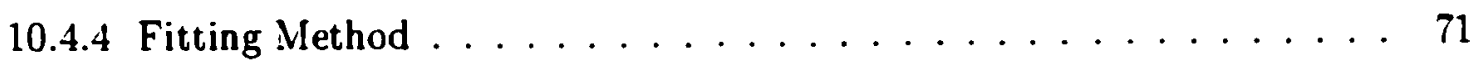

10.4.5 Signal Cross Checks $\ldots \ldots \ldots \ldots \ldots \ldots \ldots$

10.5 Measurement of $b \bar{b}$ Fraction . . . . . . . . . . . 79

11 The Search for $B_{s} \rightarrow D_{s} \pi \quad 80$

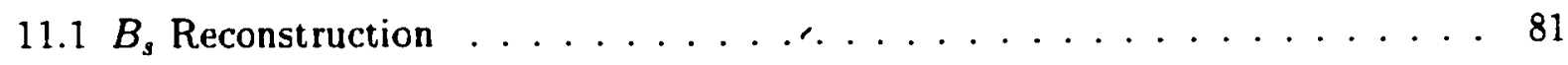


11.2 Optimization Procedure $\ldots \ldots \ldots \ldots \ldots \ldots \ldots \ldots$

$11.3 B_{s}^{0} \rightarrow D_{s}^{-} \pi^{+}$Candidates $\ldots \ldots \ldots \ldots \ldots \ldots \ldots$

11.3.1 $B_{s}^{0} \rightarrow D_{s}^{-} \pi^{+}, D_{s}^{-} \rightarrow \phi \pi^{-}, \phi \rightarrow K^{+} K^{-} \ldots \ldots \ldots \ldots \ldots$

11.3.2 $B_{s}^{0} \rightarrow D_{s}^{-} \pi^{+}, D_{s}^{-} \rightarrow \phi \pi^{-} \pi^{-} \pi^{+}, \phi \rightarrow K^{+} K^{-} \ldots \ldots \ldots \ldots 87$

11.3.3 $B_{s}^{0} \rightarrow D_{s}^{-} \pi^{+}, D_{s}^{-} \rightarrow K^{\bullet^{0}} K^{-}, K^{\bullet^{0}} \rightarrow K^{+} \pi^{-} \ldots \ldots \ldots 88$

11.3.4 $B_{s}^{0} \rightarrow D_{s}^{-} \pi^{+}, D_{s}^{-} \rightarrow K_{s} K^{-}, K_{s} \rightarrow \pi^{+} \pi^{-} \ldots \ldots \ldots \ldots$

11.3.5 $\quad B_{s}^{0} \rightarrow D_{s}^{-} \pi^{+}, D_{s}^{-} \rightarrow \overline{f_{0}}(980) \pi^{-}, \overline{f_{0}}(980) \rightarrow \pi^{+} \pi^{-} \ldots \ldots \ldots 90$

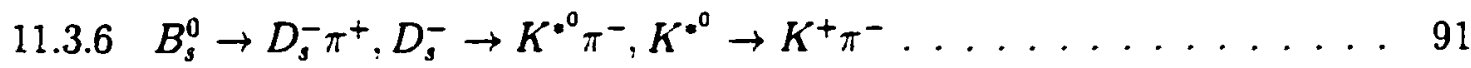

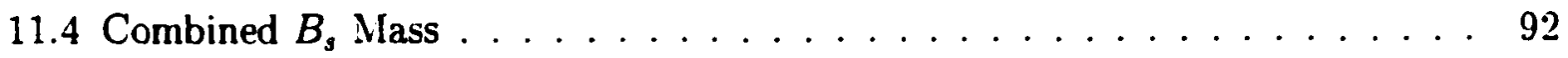

11.5 Average Selection . . . . . . . . . . . . . . . . . 93

$\begin{array}{lr}12 \text { Branching Ratio Limit } & 97\end{array}$

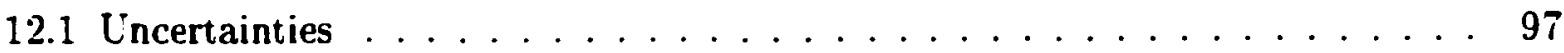

12.2 Limit Studies $\ldots \ldots \ldots \ldots \ldots \ldots \ldots \ldots \ldots$

12.2.1 Comparison of Modes $\ldots \ldots \ldots \ldots \ldots \ldots \ldots$

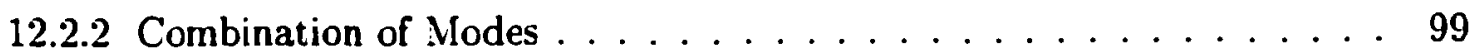

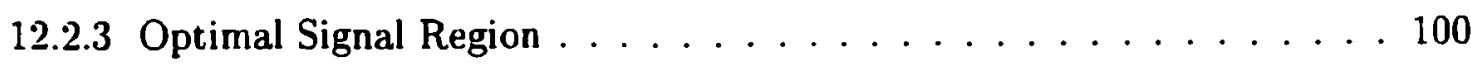

$12.3 B_{s}^{0} \rightarrow D_{s}^{-} \pi^{+}$Branching Ratio Upper Limit $\ldots \ldots \ldots \ldots 10 \ldots \ldots$

12.3.1 Upper Limit Without Uncertainties $\ldots \ldots \ldots \ldots \ldots$. . . . . 102

12.3.2 Upper Limit with Systematic and Background Uncertainties . . . . . 103 


\section{List of Figures}

1 A Feynman diagram representing the $\mathrm{e}^{+} \mathrm{e}^{-} \rightarrow \mu^{+} \mu^{-}$process. $\ldots \ldots .9$

2 The charged current Feynman diagram representing $d$-quark to $u$-quark tran-

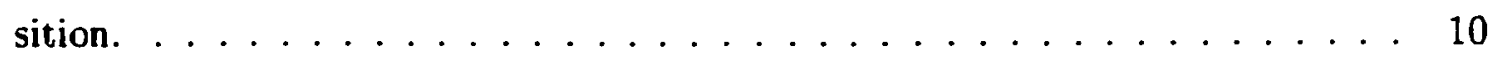

3 The unitarity triangle representing the $\mathrm{V}_{\mathrm{CKM}}$ matrix elements. $\ldots \ldots \ldots 13$

4 The leading order Feynman diagrams depicting $b$-quark production by quark anti-quark annihilation and gluon-gluon fusion. . . . . . . . 15

$j \quad$ Some Feynman diagram depicting $\mathrm{O}\left(\alpha_{s}^{3}\right)$ production mechanisms of b-quark

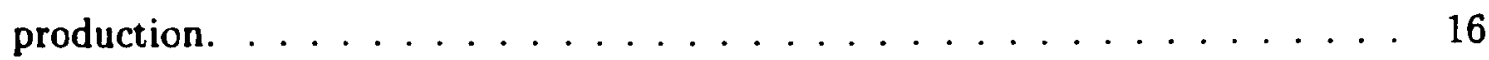

6 The Feynman diagram for $B \rightarrow D \pi$ decay. . . . . . . . . . . . 19

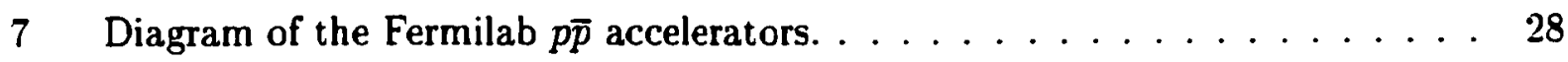

8 A cross sectional view of a quarter of the CDF detector. $\ldots \ldots \ldots 32$

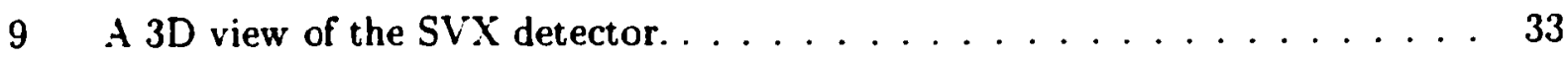

10 A schematic of the $\operatorname{VTX}$ detector. $\ldots \ldots \ldots \ldots \ldots \ldots$

11 The Central Tracking Chamber endplate. . . . . . . . . . . . 41

12 A schematic of the CMU modules. . . . . . . . . . . . 42

13 A schematic of the $b \bar{b}$ events of interest. One $B$ meson decays to $D \pi$ while

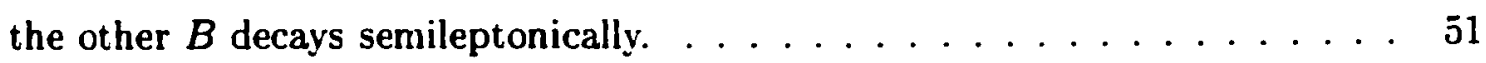

14 The reconstructed mass for $\bar{D}^{0} \rightarrow K^{+} \pi^{-}$Monte Carlo events. . . . . . . 62

15 The reconstructed mass for $D^{-} \rightarrow K^{+} \pi^{-} \pi^{-}$Monte Carlo events. . . . . 63 
16 The reconstructed mass for $B^{0} \rightarrow D^{-} \pi^{+}$Monte Carlo events. . . . . . . . 6 64

17 The reconstructed mass for $B^{+} \rightarrow \vec{D}^{0} \pi^{+}$Monte Carlo events. . . . . . 65

18 Monte Carlo distribution of $B$ and $D$ mesons proper decay time in $B^{+} \rightarrow$ $\overline{D^{0}} \pi^{+}, \overline{D^{0}} \rightarrow K^{+} \pi^{-}$and $B^{0} \rightarrow D^{-} \pi^{+}, D^{-} \rightarrow K^{+} \pi^{-} \pi^{-}$decay modes. The top plot is the Monte Carlo proper decay time distribution of $B$ mesons. The bottom left plot is the Monte Carlo proper decay time distribution of the $\overline{D^{0}}$ meson. The bottom right plot is the Monte Carlo proper decay time distribution of the $D^{-}$meson $\ldots \ldots \ldots \ldots \ldots \ldots 6$

19 Definition of the cone around the $B$ meson used in the isolation calculation. . 67

20 Wonte Carlo distribution of the isolation variable in $B^{+} \rightarrow \overline{D^{0}} \pi^{+}, \overline{D^{0}} \rightarrow$ $K^{+} \pi^{-}$and $B^{0} \rightarrow D^{-} \pi^{+}, D^{-} \rightarrow K^{+} \pi^{-} \pi^{-}$decay. $\ldots \ldots \ldots 68$

21 The Monte Carlo mass distribution of $B \rightarrow D^{(*)} \pi$ candidates reconstructed in $B^{+} \rightarrow \overline{D^{0}} \pi^{+}, \overline{D^{0}} \rightarrow K^{+} \pi^{-}$and $B^{0} \rightarrow D^{-} \pi^{+}, D^{-} \rightarrow K^{+} \pi^{-} \pi^{-}$modes. The left peak represents the $B \rightarrow D^{*} \pi$ decays (reconstructed as $B \rightarrow D \pi$ ) and the

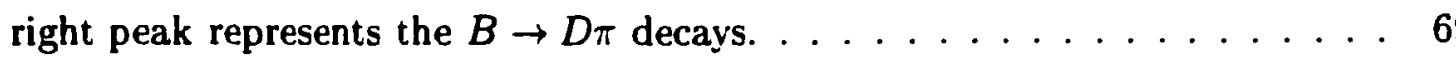

22 These four plots show the mass of the reconstructed $B$ meson candidates. The top row shows mass of $B^{+} \rightarrow \overline{D^{0}} \pi^{+}, \overline{D^{0}} \rightarrow K^{+} \pi^{-}$candidates for electron (left) and muon (right). The bottom row shows the mass of $B^{0} \rightarrow D^{-} \pi^{+}, D^{-} \rightarrow$ $K^{+} \pi^{-} \pi^{-}$candidates. The solid histogram represents $B$ candidates in the signal region and the dashed histogram represents $B$ candidates outside the signal region. $\ldots \ldots \ldots \ldots \ldots \ldots \ldots \ldots \ldots \ldots \ldots$ 
23 The combined mass of the $B^{0}$ and $B^{+}$candidates for electron and muon data. The Gaussian and linear function plotted on the data use the parameters returned from the the extended log-likelihood fit. The width of the Gaussian is equal to the average uncertainty in the mass fit scaled by a factor of 1.3 . The solid histogram represents $B$ candidates in the signal region and the dashed histogram represents $B$ candidates outside the signal region.

24 These four plots show the normalized mass of the $B$ candidates. The top row shows the normalized mass of the $B^{+} \rightarrow \overline{D^{0}} \pi^{+}, \overline{D^{0}} \rightarrow K^{+} \pi^{-}$events for electron (left) and muon (right) events. The bottom row shows the normalized mass of the $B^{0} \rightarrow D^{-} \pi^{+}, D^{-} \rightarrow K^{+} \pi^{-} \pi^{-}$events. . . . . . . . 75

25 The combined normalized mass of the electron and muon $B^{+} \rightarrow \overline{D^{0}} \pi^{+}, \overline{D^{0}} \rightarrow$ $K^{+} \pi^{-}$and $B^{0} \rightarrow D^{-} \pi^{+}, D^{-} \rightarrow K^{+} \pi^{-} \pi^{-}$candidates. $\ldots \ldots \ldots 76$

26 The proper decay time of the combined $B^{+}$and $B^{0}$ candidates. . . . . 77

27 The proper decay length of the combined $D^{+}$and $\bar{D}^{0}$ candidates. . . . . 78

28 The reconstructed Monte Carlo mass of $B_{\mathrm{s}}$ in all mode. . . . . . 83

29 The mass of $B_{s}$ candidates in the $B_{s}^{0} \rightarrow D_{s}^{-} \pi^{+}, D_{s}^{-} \rightarrow \phi \pi^{-}, \phi \rightarrow K^{+} K^{-}$mode. The shaded region denotes a $\pm 3 \sigma$ region around the nominal $B_{s}$ mass. . . . 86

30 The mass of $B_{s}$ candidates in the $B_{s}^{0} \rightarrow D_{s}^{-} \pi^{+}, D_{s}^{-} \rightarrow \phi \pi^{-} \pi^{-} \pi^{+}, \phi \rightarrow K^{+} K^{-}$ mode. The shaded region denotes a $\pm 3 \sigma$ region around the nominal $B_{s}$ mass. 87 
31 The mass of $B_{s}$ candidates in the $B_{s}^{0} \rightarrow D_{s}^{-} \pi^{+}, D_{s}^{-} \rightarrow K^{{ }^{0}} K^{-}, K^{*^{0}} \rightarrow$ $K^{+} \pi^{-}$mode. The shaded region denotes a $\pm 3 \sigma$ region around the nominal

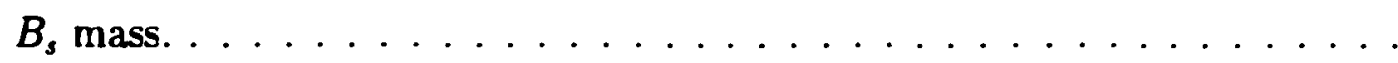

32 The mass of $B_{s}$ candidates in the $B_{s}^{0} \rightarrow D_{s}^{-} \pi^{+}, D_{s}^{-} \rightarrow K_{s} K^{-}, K_{s} \rightarrow \pi^{+} \pi^{-}$mode. The shaded region denotes a $\pm 3 \sigma$ region around the nominal $B_{s}$ mass.

33 The mass of $B_{s}$ candidates in the $B_{s}^{0} \rightarrow D_{s}^{-} \pi^{+}, D_{s}^{-} \rightarrow \overline{f_{0}}(980) \pi^{-}, \overline{f_{0}}(980) \rightarrow$ $\pi^{+} \pi^{-}$mode. The shaded region denotes a $\pm 3 \sigma$ region around the nominal $B_{s}$ mass. . . . . . . . . . . . . . . . . . . 90

34 The mass of $B_{s}$ candidates in the $B_{s}^{0} \rightarrow D_{s}^{-} \pi^{+}, D_{s}^{-} \rightarrow K^{{ }^{0}} \pi^{-}, K^{0^{0}} \rightarrow K^{+} \pi^{-}$mode. The shaded region denotes a $\pm 3 \sigma$ region around the nominal $B_{s}$ mass. . . . 91

35 The six plot shown above are the six $B_{s}^{0} \rightarrow D_{s}^{-} \pi^{+}$decay modes. The shaded region denotes a $\pm 3 \sigma$ region around the nominal $B_{s}$ mass. . . . . . . 92

36 The combined mass plot for all $B_{s}^{0} \rightarrow D_{s}^{-} \pi^{+}$decay modes. The shaded region is the $\pm 3 \sigma$ signal mass region. . . . . . . . . . . . . 94

37 The mass plot for all $B_{s}^{0} \rightarrow D_{s}^{-} \pi^{+}$decay modes with the AVERAGE selection criteria. The shaded region is the $\pm 3 \sigma$ signal mass region. . . . . . . . 95

38 The combined mass plot for all $B_{s}^{0} \rightarrow D_{s}^{-} \pi^{+}$decay modes with the AVERAGE selection criteria. The shaded region is the $\pm 3 \sigma$ signal mass region. . . . . . 96 


\section{List of Tables}

1 The mass and charge of the quarks in the Standard Model. . . . . . . . . 4

2 The mass and charge of the leptons in the Standard Model. . . . . . . . j

3 The range of values and step size for each variable used in the optimization.

The $B^{ \pm}$optimized selection criteria are found in the last column.. . . . . . 60

4 The range of values and step size for each variable used in the optimization.

The $B^{0}$ optimized selection criteria are found in the last column. . . . . 61

$\overline{5}$ The $b \bar{b}$ fraction in the inclusive lepton dataset. ........... 81

6 The range of values and step size for each variable used in the optimization.

The $B_{s}$ optimized selection criteria are found in the last column. . . . . . 85

7 The optimization cuts for each $B_{s} \rightarrow D_{s} \pi$ decay mode. The $D_{s} \pi$ decay mode modes are identified in the first column. The last row contains the average

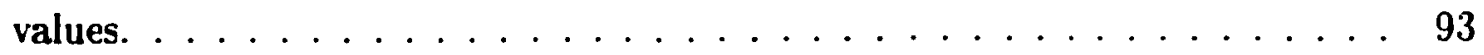

8 Each of the systematic uncertainties in the components of the scaling factor are listed as well as the total systematic error. . . . . . . . . . . . . 98

9 The sensitivity of each $B_{s} \rightarrow D_{s} \pi$ decay mode is found in the last column.

The $D_{\mathrm{s}}$ modes are listed in the second column. . . . . . . . . . . 99

10 The sensitivity for different combination of $B_{s} \rightarrow D_{s} \pi$ decay modes. . . . 100 
11 The sensitivity for two definitions of the signal region around the $B_{s}$ mass. Top top row lists the sensitivity for a $\pm 2 \sigma$ window and the bottom row lists the sensitivity for $a \pm 3 \sigma$ window. $\ldots \ldots \ldots \ldots \ldots \ldots$

12 This table shows the background uncertainty extrapolated from fit of background to signal region. $\ldots \ldots \ldots \ldots \ldots \ldots \ldots \ldots$

xviii 


\section{Part I}

\section{Theory}

\section{Introduction}

To date, the Standard Model is the most complete theory describing the fundamental particles and interactions which make up our universe. However, despite its many successes, questions persist about its ability to comprehensively describe all of nature's pheonomena. Measurements of the Standard Model's parameters check its internal consistency and possibly lead to the observation of physics beyond the Standard Model. The study of $b$-physics provides an arena for measuring some of these parameters, specifically the CKM matrix elements and $\mathrm{CP}$-violating parameters. There are several $B$ meson measurements that potentially yield information on the CKM matrix elements, for example, the observation of $B_{s}^{0}-\bar{B}_{s}^{0}$ mixing. The ability to fully reconstruct the $B_{s}$ meson in mixing studies offers the most precise measurements of its proper decay time, crucial to the observation of $B_{s}^{0}-\overline{B_{s}^{0}}$ mixing for large $\Delta m$. This thesis studies two-body fully hadronic $B$ meson decays through the Cabbibo favored $b \rightarrow c$ transition and places an upper limit on the $B_{s}^{0} \rightarrow D_{s}^{-} \pi^{+}$branching ratio ${ }^{1}$

This analysis is performed on data collected by the Collider Detector at Fermilab (CDF) at the Fermi National Accelerator Laboratory from 1992-1995, referred to as Run I. The

\footnotetext{
${ }^{1}$ Charge conjugation is assumed throughout.
} 
Run I $110 \mathrm{pb}^{-1}$ inclusive lepton dataset is used to search for $B \rightarrow D \pi$ decays. The reference signal used in this analysis requires the reconstruction of $B^{+}$and $B^{0}$ mesons via their charmed hadronic decay products. These $B$ mesons are reconstructed in the hemisphere opposite the lepton's hemisphere. After identifying the reference signal, six $B_{s} \rightarrow D_{s} \pi$ decay modes are searched for. An upper limit on the $B_{s} \rightarrow D_{s} \pi$ decay rate is calculated based on the number of reconstructed $B^{+}$and $B^{0}$ mesons, the relative reconstruction efficiencies and branching ratios, the $b$-quark fragmentation fractions and an upper limit on the number of $B_{s}$ events.

An introduction to the theoretical background and the motivation for this study is presented in Sections 2, 3 and 4 . A description of the experimental apparatus is presented in Sections 5 and 6 . The goals of this analysis are outlined in Section 7 . The data sample and strategy for finding $B$ mesons is presented in Section 8. The reconstruction of $B^{+}$and $B^{0}$ mesons is detailed in Section 9. The optimization of the $B^{+}$and $B^{0}$ reference signal is found in Section 10. The search for $B_{s}$ mesons is explained in Section 11. The upper limit on the $B_{s} \rightarrow D_{s} \pi$ branching ratio is calculated in Section 12. Finally, conclusions are discussed in Section 13. 


\section{Standard Model}

The Standard Model describes fundamental particles and their interactions based on the requirement of local gauge invariance. The Standard Model is composed of Quantum Chromodynamics (QCD) and Electroweak $(\mathrm{GSW})^{2}$ gauge theories. Gravity is not included in the Standard Model and is best described by Albert Einstein's General Relativity Theory since the gravitational force, at the current energy scale, is negligible at the atomic and sub-atomic levels,

The Standard Model groups particles into half-integer spin (fermions) and integer spin (bosons) categories. Fermions are composed of either quarks or leptons. The six flavors of quarks are divided into three generations of doublets and are shown in Table 1 [1]. However, quarks are not found individually in nature. Instead, they are paired as quark anti-quark pairs, called mesons, or quark triplets, known as baryons. Mesons and baryons are collectively classified as hadrons. Leptons are also grouped into three generational doublets and are shown in Table 2 [1]. Leptons can be found individually in nature.

Bosons are integer spin particles which obey Bose-Einstein statistics. The bosons serve as the gauge fields of the previously mentioned gauge groups and mediate particle interactions. The $\mathrm{W}$ and $\mathrm{Z}$ bosons mediate the weak force, gluons mediate the strong force, and photons mediate the electromagnetic force. In the following sections, the historical development and construction of the theories which constitute the Standard Model are summarized.

\footnotetext{
"Named for Glashow, Weinberg and Salaam who unified the weak interaction with Quantum Electrodynamics (QED).
} 


\begin{tabular}{|c|c|c|}
\hline \hline Quark & Charge & Mass $\left[\mathrm{GeV} / \mathrm{c}^{2}\right]$ \\
\hline \hline up & $+2 / 3$ & $0.001-0.005$ \\
down & $-1 / 3$ & $0.003-0.009$ \\
\hline charm & $+2 / 3$ & $1.15-1.35$ \\
strange & $-1 / 3$ & $0.075-0.170$ \\
\hline top & $+2 / 3$ & $4.0-4.4$ \\
bottom & $-1 / 3$ & $174.3 \pm 5.1$ \\
\hline \hline
\end{tabular}

Table 1: The mass and charge of the quarks in the Standard Mlodel.

\subsection{History}

The multitude of particle discoveries during the last century began with JJ Thompson's discovery of the electron in 1897. This established that the atom, once thought to be a basic "building block" of nature, was in fact made of more fundamental pieces of matter. Albert Einstein followed Thompson's discovery by suggesting that electromagnetic radiation consisted of photons. Based on this, Einstein proposed the theory of the photoelectric effect. The examination of the atom's substructure continued when Ernest Rutherford, in 1911, discovered the presence of a positively charged nucleus in an atom, by colliding $\alpha$-particles ${ }^{3}$ with thin gold foils and observing that some deflected at large angles. These positively charged particles were known as protons. The makeup of the atomic nucleus was completed in 1932 when James Chadwick discovered neutrons.

Though these initial discoveries answered some questions, they also introduced many new

\footnotetext{
${ }^{3}$ Helium nuclei.
} 


\begin{tabular}{|c|c|c|}
\hline \hline Lepton & Charge & Mass $\left[\mathrm{MeV} / c^{2}\right]$ \\
\hline \hline $\mathrm{e}$ & -1 & 0.511 \\
$\nu_{e}$ & 0 & $<3.0 \times 10^{-6}$ \\
\hline$\mu$ & -1 & 105.6 \\
$\nu_{\mu}$ & 0 & $<0.19(90 \%$ C.L. $)$ \\
\hline$\tau$ & -1 & 1777.0 \\
$\nu_{\tau}$ & 0 & $<18.2(95 \%$ C.L. $)$ \\
\hline \hline
\end{tabular}

Table 2: The mass and charge of the leptons in the Standard Model.

ones. For example, since it was not possible for the electromagnetic force to account for the bound state of the nucleus, an unknown attractive force must be responsible. This unknown force was thought to act on a very short range $\left(10^{-12} \mathrm{~cm}\right)$ and be strong enough to overcome the electromagnetic repulsion of the protons. In 1934, Yukawa proposed that a force existed between protons and neutrons that was mediated by a massive boson. The boson's mass would explain why the force only acted over a short distance (on the scale of the atomic nucleus).

In addition to questions about the unknown attractive force, it was believed that a unknown force must also be acting in $\beta$-decay. During $\beta$-decay, a neutron decays to a proton and an electron. However, the mean decay time of $\beta$-decay was not easily explainable by the attractive or electromagnetic force. Thus, a third force was theorized to exist. In addition, the energy spectrum of the emitted electrons was continuous up to the maximum available energy. To account for this, a nearly massless neutrino, $\nu$, was postulated by Wolfgang Pauli 
in 1930 , to be an invisible third particle present in the decay: $n \rightarrow p e^{-} \overline{\nu_{e}}$. In 1938, the muon was discovered by Carl David Anderson in a cosmic ray experiment. In a process similar to B-decay, the muon also decays $\mu \rightarrow e^{-} \bar{\nu}_{e} \nu_{\mu}$.

In the middle of the last century there were many unanswered questions including newly discovered but unexplained forces and particles. With the introduction of particle accelerators in the $1950^{\prime}$ 's, many elementary' previously unknown particles were being created and observed. For example, the pion was discovered in 1947. As the number of 'elementary' particles increased and the symmetries in mass, charge and intrinsic spin between these particles were observed, doubts were raised that these were truly 'elementary' particles. In 1963, Murray Gell-Mann and George Zweig proposed that hadrons were in fact made of smaller, more fundamental, particles they called quarks. Gell-Mann and Zweig's model consisted of three quarks (up, down and strange) which interacted via the strong force. The theory of a further layer of structure beneath the nucleon scale was proven correct with the discovery of proton substructure in 1968 at the Stanford Linear Accelerator Center (SLAC).

To explain the large decay time of 3-decay and parity and flavor violation, a new interaction was needed. Either a new field with its own coupling strength or a new field with the same strength as the electromagnetic field but with a mediator that had a large mass. Gell-Mann and Kazuhito Nishijma attributed this to a new quantum quantity called 'strangeness' which would be conserved in strong and electromagnetic interactions but not in weak interactions. However, the strangness changing decays observed were inconsistent with the current $\mathrm{SU}(3)$ symmetry believed to explain the three quark flavors. To fix this problem Nicola Cabbibo, in 1963, suggested that the charged weak interaction eigenstates 
were not the mass eigenstates, but linear combinations of them. In 1970, Glashow, Iliopouls and Maiani predicted the existence of a fourth quark, charm, to solve the problem. In 1973 , Kobayashi and Maskawa proposed a new generation of quarks to solve the problem of $\mathrm{CP}$ violation [2]. The charm quark prediction was confirmed the following year in 1974, when two separate experiments led by Samuel Ting and Burton Richter discovered the $c \bar{c}$-bound state named the $\mathrm{J} / \mathrm{\psi}$.

The discoveries of fundamental particles continued with Martin Perl's discovery of the $\tau$ lepton in 1975 at SLAC. The fifth quark, bottom, was discovered as a resonance in the dimuon invariant mass spectrum by a group led by Leon Lederman in 1977 at Fermilab [3]. The last quark, top, was discovered at Fermilab by CDF and D0 in 1995 [4] [5]. Recently, experimental evidence for neutrino mass has been presented [6]. Based on this abbreviated history of the development of particle physics the underlying theories of the Standard Model are explored.

\subsection{The Standard Model}

The Standard Model breaks down all matter in the universe to basic fundamental particles and three basic interactions. The interactions between these particles are all dictated by local gauge invariance. The next three sections describe these interactions, Quantum Electrodynamics, Quantum Chromodynamics, and Electroweak, which constitute the Standard Model. 


\subsubsection{Quantum Electrodynamics}

Quantum Electrodynamics (QED) is the gauge theory of electromagnetism. This theory couples Maxwell's classical electromagnetic equations with the modern formalism of quantum field theory. It is a renormalizable field theory based on the concept of local gauge symmetry. Renormalizable QED was the first successful quantum field theory.

The QED field is mediated by the massless photon. The gauge symmetry asserts that the photon couple to all electrically charged matter with a strength proportional to the electric charge. Because QED is gauge invariant, electric charge must be conserved in all electromagnetic interactions. A Feynman diagram representing a basic QED process, electron-positron annihilation, is shown in Figure 1. A electron and positron annihilate to from a photon that produces a muon and anti-muon pair. The amplitudes are described by the interaction vertices for the emission or absorption of photons. The vertices contribute a factor proportional to the fine structure constant, $\alpha$, to the amplitude of the process. Because $\alpha$ is small, diagrams with an increased number of vertices representing more complex processes contribute less to the final magnitude of the process.

\subsubsection{Quantum Chromodynamics}

QCD is the local gauge theory of the strong interaction, based on the non-Abelian SC(3) gauge theory. The fact that $\mathrm{QCD}$ is non-Abelian leads to phenomena known as asymptotic freedom and infrared slavery. QCD is mediated by gluons that have a quantum similar to electric charge, known as color, that has three fundamental values. QCD requires eight massless spin-1 gauge bosons, corresponding to the different ways they can couple to the 


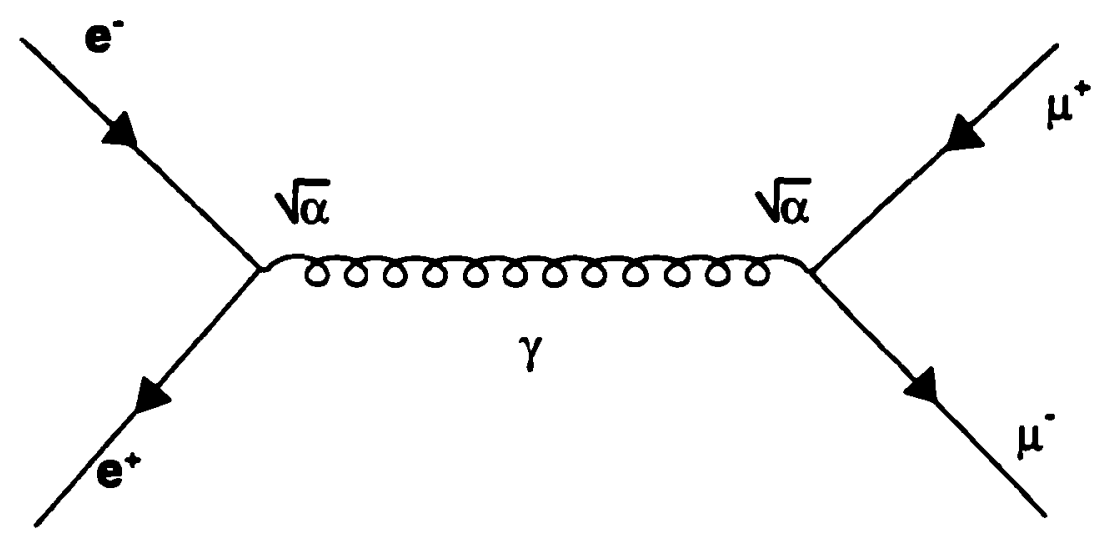

Figure 1: A Feynman diagram representing the $\mathrm{e}^{+} \mathrm{e}^{-} \rightarrow \mu^{+} \mu^{-}$process.

three colors of quarks. However, gluons are also colored and therefore interact with each other, unlike photons. This leads to additonal vertices in QCD that are not found in QED. QCD interactions are diagonal in quark flavors, that is, they do not change the flavor of a quark. Also, because leptons have no color, gluons do not couple to them.

\subsubsection{Electroweak}

The electroweak force lies at the heart of $B$ hadron decay, since if there were no cross generational transitions, the $B^{0}$ meson, the lightest $B$ meson, would not decay at all. The electroweak force is a spontaneously-broken non-Abelian local gauge invariance based on the 
group $\mathrm{SC}^{-}(2)_{L} \times \mathrm{C}(1)$. After spontaneous symmetry breaking, the four massless bosons give rise to three massive spin- 1 bosons, $W^{ \pm}$and $Z$, which mediate the $V-A$ structure of the weak force and a massless spin- 1 boson, $\%$, which mediates the electromagnetic force. A Feynman diagram representing the charged current interaction in which the $\mathrm{W}$ boson mediates the $d$-quark to $u$-quark transition shown in Figure 2. A further spin-0 boson, called the Higgs boson, arises in spontaneous symmetery breaking.

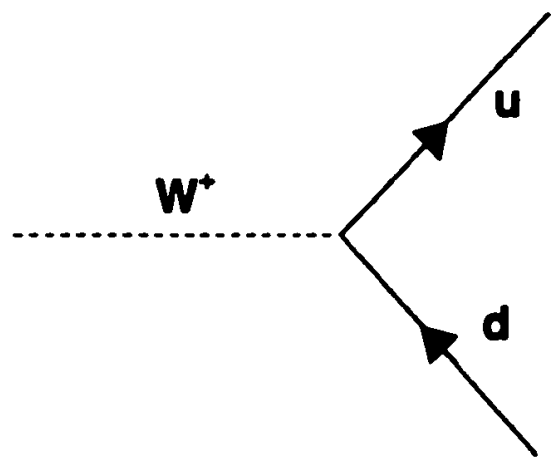

Figure 2: The charged current Feynman diagram representing $d$-quark to $u$-quark transition. 


\subsubsection{CKM Matrix}

The six quarks are arranged into doublets based on their weak isospin. Quarks in one generation may mix with quarks in other generations. The weak force couples the pairs:

$$
\left(\begin{array}{l}
u \\
d^{\prime}
\end{array}\right),\left(\begin{array}{l}
c \\
s^{\prime}
\end{array}\right),\left(\begin{array}{l}
t \\
b^{\prime}
\end{array}\right)
$$

The Cabibbo-Kobayashi-.Maskawa (CKM) matrix relates the weak interaction eigenstates to the quark mass (physical) eigenstates. The CKM matrix, $\vee_{\mathrm{CKM}}$, is a $3 \times 3$ unitary matrix. in the quark flavor space:

$$
\left(\begin{array}{c}
d^{\prime} \\
s^{\prime} \\
b^{\prime}
\end{array}\right)=\left(\begin{array}{ccc}
V_{u d} & V_{u s} & V_{u b} \\
V_{c d} & V_{c s} & V_{c b} \\
V_{t d} & V_{t s} & V_{t b}
\end{array}\right)\left(\begin{array}{l}
d \\
s \\
b
\end{array}\right)
$$

where $d^{\prime}, s^{\prime}, b^{\prime}$ are linear combinations of the physical quarks $d, s, b$. Every weak interaction involving the $W$ boson is proportional to some product of the elements of $V_{C K M}$. Because the matrix is unitary, the 18 free parameters of a non-unitary $3 \times 3$ matrix reduce to nine free parameters. After applying five quark phase adjustments, the number of free parameters is reduced to three amplitudes and one phase. The Wolfenstein parameterization approximates the matrix elements and provides a good description of the magnitude and the relationship of those elements with large phases [7]:

$$
V_{C K M} \cong\left(\begin{array}{ccc}
1-\frac{1}{2} \lambda^{2} & \lambda & A \lambda^{3}(\rho-i \eta) \\
-\lambda & 1-\frac{1}{2} \lambda^{2} & A \lambda^{2} \\
A \lambda^{3}(1-\rho-i \eta) & A \lambda^{2} & 1
\end{array}\right) .
$$


In this approximation, $\lambda=\sin \theta_{c}=0.22$, where $\theta_{c}$ is the Cabbibo angle [8]. The parameters $\rho$ and $\eta$ contain the phase information. The unitarity requirement forces the sum of the absolute squares of the elements in any row to equal one. It also requires that any pair of rows or columns of the matrix be orthogonal. The expression most relevant to $B$ physics is:

$$
V_{u d} V_{u b}^{*}+V_{c d} \dot{V}_{c b}^{\ddot{*}}+V_{t d} V_{t b}=0
$$

This constraint is commonly represented by a triangle in the complex plane after rescaling the sides by $\left|V_{c d} V_{c b}^{* b}\right|$ as shown Figure 3. The angles can be measured and related to an observation of $\mathrm{CP}$ violation:

$$
\begin{gathered}
\tan (\alpha)=\frac{\eta}{\left(\eta^{2}-\rho(1-\rho)\right)} \\
\tan (3)=\frac{\eta}{(1-\rho)} \\
\tan (\gamma)=\frac{\eta}{\rho}
\end{gathered}
$$

\subsubsection{CP Violation}

Symmetry is one of the most important concepts in particle physics. The electroweak interaction is a consequence of the symmetry requirement of local gauge invariance. A parity transformation, $\mathrm{P}$, is equivalent to looking at physics in a mirror. Strong and electromagnetic interactions are invariant under parity transformations, however, weak interactions are not. In 1954, it was discovered that neutrinos are exclusively left-handed (spinning anticlockwise about their direction of motion) and anti-neutrinos are right-handed. Thus, both $\mathrm{C}$ and $\mathrm{P}$ are separately violated in weak interaction. In 1964 it was experimentally established that 


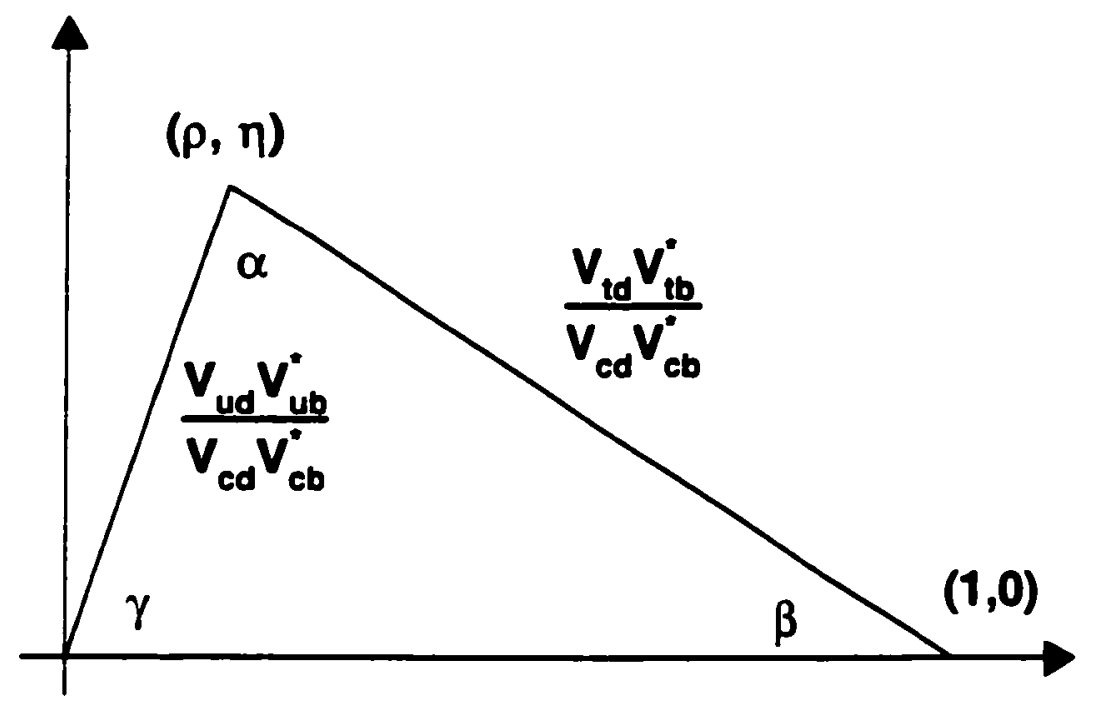

Figure 3: The unitarity triangle representing the $V_{\mathrm{CKM}}$ matrix elements.

$\mathrm{CP}$ is violated in the $\mathrm{K}^{0}$ system. In 1968, Salam, Glashow and Weinberg introduced $\mathrm{C}$ and $\mathrm{P}$ violation into the electroweak theory. Recently, BaBar and Belle have measured $\mathrm{CP}$ violation in the the $B$ system[9][10].

\section{$3 \quad b \bar{b}$ Production}

In this section, a brief introduction to $b$-quark production in $p \bar{p}$ collisions is discussed. $b \bar{b}$ pairs are produced by collision between partons ${ }^{4}$ inside the proton and anti-proton beams.

${ }^{4} Q u a r k s$ or gluons. 
The $b \bar{b}$ cross section is large at $p \bar{p}$ colliders compared to $e^{+} e^{-}$machines, however, the cross section is small compared to the total cross section in $p \bar{p}$ collisions:

$$
\frac{\sigma_{b \bar{b}}}{\sigma_{\text {total }}}=6 \times 10^{-4}
$$

This difference in cross section means that a study of $B$ hadrons must efficiently select the $b \bar{b}$ events of interest from the majority of events. There are three main processes which occur during the proton anti-proton collision. These are $b \bar{b}, c \bar{c}$, and light quark/gluon processes. The $b \bar{b}$ production cross section can be expressed as a pertubative series expanded in powers of coupling constant, $\alpha_{s}$. First, a discussion of heavy quark production in lowest order is presented, followed by the next to lowest order QCD calculations. This followed by an introduction to the fragmentation of $B$ hadrons.

\subsection{Leading Order}

The lowest order $b \bar{b}$ production processes are called s-channel and t-channel and are of order $\alpha_{s}^{2}$. The t-channel mechanism is a $2 \rightarrow 2$ process of gluon-gluon fusion $(g+g \rightarrow b+\bar{b})$ and a s-channel mechanism is a quark anti-quark or gluon gluon annihilation process $(q+\bar{q} \rightarrow$ $b+\bar{b}, g+g \rightarrow b+\bar{b})$. These diagrams are shown in Figure 4. At leading order, $\alpha_{s}^{2}$, the $b \bar{b}$ pairs move in opposite azimuthial directions in the parton-parton center-of-mass frame and thus are back-to-back in the transvserse plane. The $b \bar{b}$ cross section is flat at small pseudorapidity, $\eta$, where $\eta=-\ln [\tan (\theta / 2)]$ and $\theta$ is angle to the beam direction, and falls at large $\eta$. The center-of-mass of the system is boosted along the $p \bar{p}$ axis due to partons carrying different fractions of the proton and anti-proton momentum. 

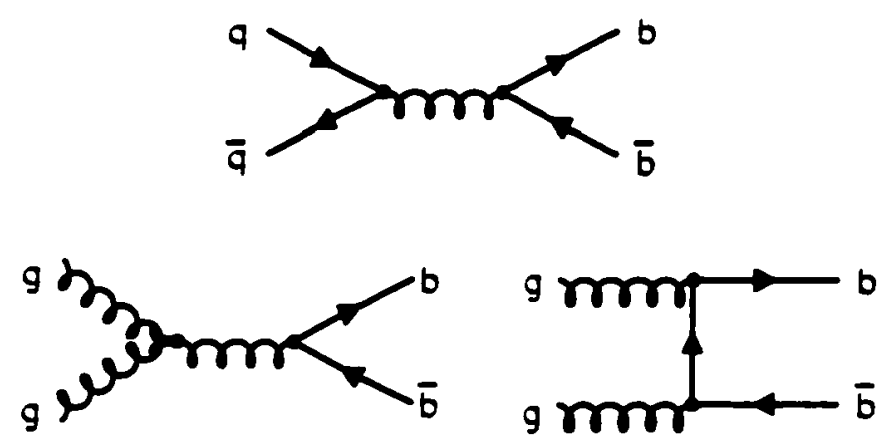

Figure 4: The leading order Feynman diagrams depicting $b$-quark production by quark antiquark annihilation and gluon-gluon fusion.

\subsection{Next To Leading Order}

There are four main types of next to leading order, NLO, production processes: gluon splitting, virtual emission, real emission and flavor excitation. The NLO processes also include initial and final state gluon radiation, producing $b \vec{b}$ pairs plus additional light quarks or gluons. Further complicating the production of $b \bar{b}$ pairs, is the underlying event that is caused by partons not participating in the $b \bar{b}$ event. Some of the Feynman diagrams representing the NLO process are shown in Figure 5 . Gluon-gluon processes, $g+g \rightarrow q+\bar{q}$, are the dominate process. These processes result in particles with low transverse momentum and high $\eta$. NLO $b \bar{b}$ pairs are usually created closer together in phase space and are not as likely as pairs from LO procesess to appear in the detectors central region. However, the higher-order terms are similar in magnitude to lowest-order, thus they are not simply higher-order corrections. Currently the theoretical predictions for the cross sections agree 
well with the shape of data cross sectons but the data is a factor of 3 higher [11] [12].
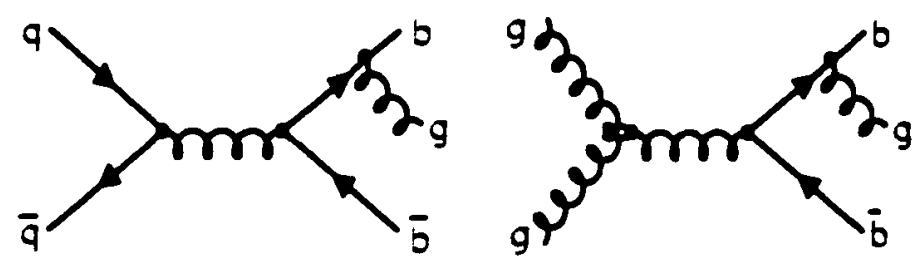

Figure 5: Some Feynman diagram depicting $\mathrm{O}\left(\alpha_{s}^{3}\right)$ production mechanisms of $b$-quark production.

\section{3 $B$ Fragmentation}

After the proton and anti-proton interaction, individual quarks exit the interaction region and hadronize to form color neutral hadrons. The individual quarks are joined by other quarks to form hadrons by pulling $q \bar{q}$ pairs out of the vacuum. This is a result of the color (strong) force between quarks. One of the quarks joins the heavy quark and forms a hadron. Fragmentation is a low $Q^{2}$, non-pertubative, $Q C D$ process. Because this is a low energy process, phenomenological models to quantify the process must be used. The fragmentation process is characterized by the fraction of the initial $b$-quark momentum, $z$, carried by the $B$ meson. The fragmentation function, $D(z)$, represents the probability distribution of the $B$ meson's momentum transverse to the $p \bar{p}$ axis. Since it is a long distance process, a semi-empirical parameterization is used [13]:

$$
D(z)=\frac{N}{z(1-1 / z-\epsilon(1-z))^{2}}
$$


where $\mathrm{N}$ is a normalization constant and

$$
z=\frac{\left(E+p_{\|}\right)_{Q \bar{q}}}{\left(E+p_{Q}\right)}
$$

and $p_{\|}$is the projection of the momentum of the hadron onto the original direction of the heavy quark. The Peterson fragmentation parameter, $\epsilon$, is related to the ratio of the heavy and light quark effective mass.

One of the models describing fragmentation is the Lund Model [14]. The Lund model assumes that fragmentation is independent of production. This is reasonable since the time scale for fragmentation is much greater then the time scale for production. A naive model suggests that as the colored particles are separated, the color field between them takes on the shape of a tube. The tubes energy is determined by its length. Thus, as the displacement increases, the energy of the system increases until finally it fragments by producing $q \bar{q}$ or $q q-\bar{q} \bar{q}$ pairs. A fraction of parent quarks's energy (momentum) is carried by the resulting heavy hadron[13]. It its theorized that a fast moving quark loses negligible energy when it picks up a light anti-quark or di-quark. Since the $s$-quark is heavier then $u$ and $d$-quarks, it is reasonable to assume that it is less likely to be produced in the fragmentation process. The first-order theoretical predictions for which flavor of quark joins the b-quark to form a $B$ hadron are:

$$
u: d: s: c=1: 1: 1 / 3: 10^{-10}
$$

CDF has measured these fragmentation fractions [15]:

$$
f_{d} / f_{u}=(84 \pm 16) \%
$$




$$
\begin{gathered}
f_{s} /\left(f_{u}+f_{d}\right)=(21.3 \pm 3.8) \%, \\
f_{\text {baryon }} /\left(f_{u}+f_{d}\right)=(12.0 \pm 4.0) \% .
\end{gathered}
$$

If it is assumed that $f_{u}+f_{d}+f_{s}+f_{\text {baryon }} \equiv 1$, then:

$$
\begin{gathered}
f_{u}=(37.6 \pm 1.5) \%, \\
f_{d}=(37.6 \pm 1.5) \%, \\
f_{s}=(16.0 \pm 2.5) \%, \\
f_{\text {baryon }}=(8.9 \pm 2.8) \% .
\end{gathered}
$$

The Particle Data Group quotes the world average values as [1]:

$$
\begin{gathered}
f_{u, d}=(38.9 \pm 1.3) \%, \\
f_{s}=(10.7 \pm 1.4) \%, \\
f_{\text {baryon }}=(11.6 \pm 2.0) \% .
\end{gathered}
$$

\section{$4 \quad B$ Mesons}

$B$ hadrons are especially interesting to study since they are the heaviest hadrons experimentally accessible $e^{5}$. The pseudo-scalar $B$ mesons are $B^{0}=(\bar{b} d), B^{+}=(\bar{b} u), B_{s}=(\bar{b} s)$ and $B_{c}$ $=(\bar{b} c)$. All of the lowest mass bound states with a $b$-quark have been observed. $B$ decays are also interesting because they have the potential to yield information on the weak mixing matrix. $B$ decay measurements hold information on five of the nine CKM elements [2][16].

\footnotetext{
${ }^{5}$ The $t$-quark decays weakly to a $b$-quark before it is able to hadronize.
} 


\section{1 $B$ Decays}

Lowest lying $B$ hadrons decay weakly with significant lifetime and hence have measurable decay length. Two-body hadronic $B$ meson decays usually occur through Cabibbo favored $b \rightarrow c$ weak transition. Studies of two-body weak decays of $B$ mesons give information on

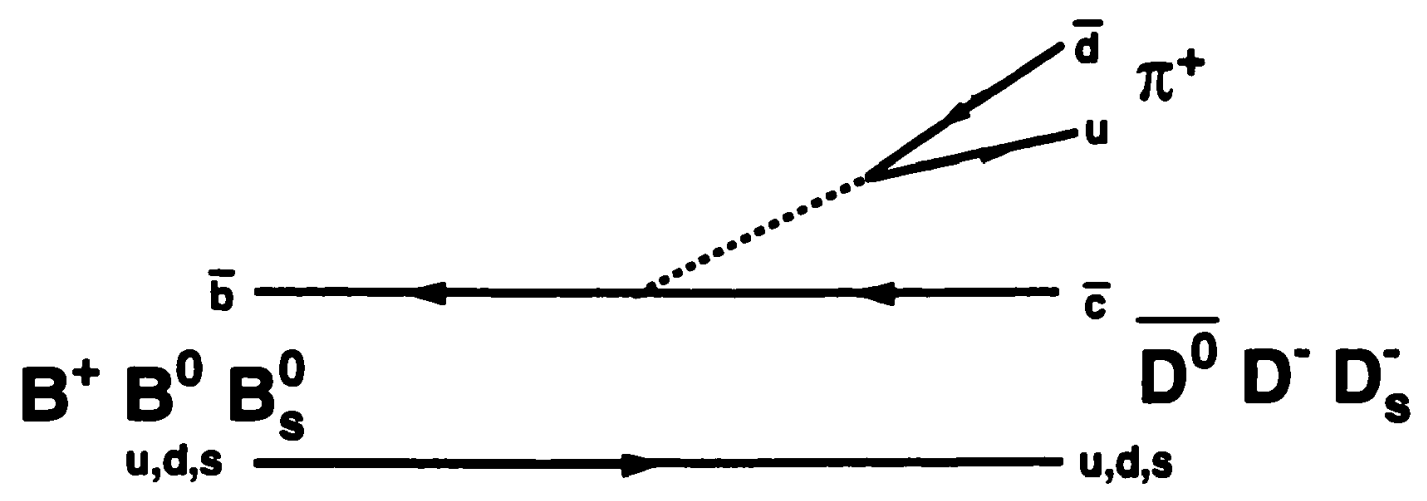

Figure 6: The Feynman diagram for $B \rightarrow D \pi$ decay.

the Standard Models parameters: $V_{q b}, \alpha_{s}$, and others [1]. The decay width for a $b$-quark decaying into a light quark is given by:

$$
\Gamma_{s l}(b \rightarrow q)=\frac{G_{F}^{2} m_{b}^{5}}{192 \pi^{3}} \times\left|V_{q b}\right|^{2} \times F(\epsilon)
$$

where $\mathrm{G}_{F}$ is the Fermi coupling constant and $\mathrm{V}_{q b}$ is the CK.M matrix element for the $b \rightarrow q$ transition. 


\subsubsection{Spectator Model}

The spectator model for a two-body weak decay of a $B$ meson is represented by a Feynman diagram in Figure 6. The spectator model dictates that the light quark remain the same flavor in the initial and final states. The dominate weak decay diagram is a spectator diagram where a virtual $\mathrm{W}^{-} \rightarrow \bar{u} d, \bar{c} s$, or $\ell \nu_{\ell}$. The quark anti-quark pair becomes one of the final state hadrons while the $\bar{c}$-quark pairs with the spectator quark to form the other hadron. The Hamiltonian for this process is:

$$
H=\frac{G_{F}}{\sqrt{2}} V_{c b}[(\bar{d} u)+(\bar{s} c)](\bar{c} b)
$$

where $\left(\bar{q}_{i} q_{j}\right)=\bar{q}_{i} \Gamma_{\mu}\left(1-\Gamma_{5}\right) q_{j}, G_{F}$ is the Fermi coupling constant and $V_{c b}$ is a CKM matrix element.

The spectator diagram is modified by hard gluon exchanges between initial and final quark lines. These effects can be taken into account by use of the renormalization group. Gluons induce an additional term so the effective hamiltonian is separated into two terms. The original hamiltonian is multiplied by the Wilson coefficent, $c_{1}(\mu)$, and an additional Hamiltonian is multiplied by a Wilson coefficent, $c_{2}(\mu)$ :

$$
H_{e f f}=\frac{G_{F}}{\sqrt{2}} l_{c b} c_{1}(\mu)[(\bar{d} u)+(\bar{s} c)](\bar{c} b)+c_{2}(\mu)[(\bar{c} u)(\bar{d} b)+(\bar{c} c)(\bar{s} b)]
$$

Wilson coefficents can be calculated from QCD. However, the calculation is difficult since its unclear at what scale these coefficents should be calculated. After setting $\mu=m_{b}^{2}{ }^{6}$, and

\footnotetext{
${ }^{6} m_{b}$ is the mass of the $b$-quark equal to $4.0-4.4 \mathrm{GeV} / c^{2}[1]$.
} 
$c_{ \pm}(\mu)=c_{1}(\mu) \pm c_{2}(\mu)$, the leading-log approximation[17] is:

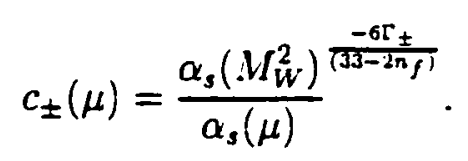

It is hoped that factorization will be a better approximation in $B$ decays then $D$ decays due to the larger energy release [18]. If factorization is valid, then Heavy Quark Effective Theory (HQET) could provide a reliable model independent framework for the calculation of properties of non-leptonic $B$ meson decays [19].

\subsubsection{Branching Ratios}

$B$ physics measurements have typically been the domain of $\mathrm{e}^{+} \mathrm{e}^{-}$machines operating at the $\Upsilon(4 S)$ or $Z^{0}$-pole. Since $B$ mesons were first fully reconstructed in 1983 by CLEO, $B_{d}$ and $B_{u}$ branching ratios have been been measured extensively and with a high degree of precision at CLEO and ARGUS [20] [21] [22]. These measurements were the product of the $\Upsilon(4 S)$ resonance in $\mathrm{e}^{+} \mathrm{e}^{-}$annihilations at CESR storage ring for CLEO and the Doris storage ring at DESY for ARGUS. Since the $\Upsilon(4 S)$ is just past the open beauty threshold, it cannot fragment into a $b \bar{b}$ final state other than $B^{+}$or $B^{0}$. Thus, CDF has the advantage of measuring $B_{s}$ decays also. This analysis relies heavily on these branching ratio measurements. The $B$ meson branching ratios used in this analysis are summarized below [1]:

$$
\begin{aligned}
& \text { - } \operatorname{BR}\left(B^{+} \rightarrow \bar{D}^{0} \pi^{+}\right)=(5.3 \pm 0.5) \times 10^{-3} \\
& \text { - } \operatorname{BR}\left(B^{0} \rightarrow D^{-} \pi^{+}\right)=(3.0 \pm 0.4) \times 10^{-3}
\end{aligned}
$$

The $D$ meson branching ratios used in this analysis are summarized below [1]: 
- $\operatorname{BR}\left(D^{-} \rightarrow K^{+} \pi^{-} \pi^{-}\right)=(9.0 \pm 0.6) \times 10^{-2}$,

- $\operatorname{BR}\left(\vec{D}^{0} \rightarrow K^{+} \pi^{-}\right)=(3.83 \pm 0.09) \times 10^{-2}$.

\section{2 $B_{s}$ Mesons}

The $B_{s}$ meson was first observed, in 1990 , by the CUSB collaboration with a collider operating at the $\Upsilon(5 \mathrm{~S})$ resonance [23]. The discovery was confirmed in 1992 by the ALEPH collaboration [24]. The $B_{s}$ meson has also been observed by the CDF collaboration via the decay channel $B_{s} \rightarrow J / \Psi \varphi[25]$ and the corresponding branching ratio was measured [26].

\subsection{1 $\quad B_{s}$ Mixing}

Information from numerous $B_{s}$ decay modes provide complimentary CKM information related to measurements involving $B^{0}$ decays. In $B_{s}^{0}-\bar{B}_{s}^{0}$ mixing, the $d$-quark is replaced with an $s$-quark. In some cases, measurements involving the ratio of $B_{s}$ to $B_{d}$ properties result in the cancellation of theoretical uncertainties. For example, a measurement of the ratio of the $B_{s}$ mixing parameter, $\Delta \mathrm{m}_{s}$, and the $B_{d}$ mixing parameter, $\Delta \mathrm{m}_{d}$, results in the cancellation of many uncertainties. In Run II, a measurement of $\Delta m_{s}$ will be combined with the well-measured $\Delta m_{d}$ to give the ratio of $\frac{\Delta m_{d}}{\Delta m_{d}}$ and a theoretically clean measurement of $\frac{\left|t_{i d}\right|^{2}}{\left|V_{t a}\right|^{2}}$ within Standard Model. This will place a precise constraint on the CKM parameters $\rho$ and $\eta[27]$. 


\subsubsection{CP Violation}

$\mathrm{CP}$ violation is one avenue to investigate the underlying theory of the Standard Model. In addition to measuring the side of the unitarity triangle, the value of $\Delta m_{s}$ can be used as a constraint in $\mathrm{CP}$ asymmetry measurements in $\mathrm{CP}$ violating $B_{s}$ decays. In the Standard Model, small CP violation is accomodated by a complex phase in the CKM matrix. However, this only means that the Standard Model allows CP violation but does not explain it. There are a variety of $B$ decay modes that potentially hold information. The more measurements which are done the better the Standard Model picture of $\mathrm{CP}$ violation is tested. Some decay modes give clean information on angles in the CKM matrix which are just the phases of various CKM elements. This information will test the hypothesis that CKMI phases causes $\mathrm{CP}$ violation. In addition, if large $\mathrm{CP}$ violating effects are observed then other 'new physics' sources of CP violation may exist other than the complex phase of the CK.M matrix.

\subsubsection{Future Prospects}

Currently lower limits on the $B_{s}^{0}-\overline{B_{s}^{0}}$ mixing frequency have been placed based on analyses using semileptonic $B_{s}$ decay modes. At CDF, the $\frac{\Delta m_{2}}{\Gamma_{1}}$ reach in semileptonic decays is expected to be about 30 for a $5 \sigma$ observation [27]. For semileptonic modes, the uncertainty in the momementum, due to the missing neutrino, is the limiting factor. However, fully reconstructed hadronic decay modes, such as $B_{s} \rightarrow D_{s}^{-} \pi^{+}$, are crucial for $B_{s}$ mixing analyses since they require excellent proper time resolution. The proper time resolution is a product of the measured $B$ meson decay length and reconstructed $B_{s}$ momentum. The uncertainty 
is given by

$$
\sigma_{t}=t \sqrt{\left(\frac{\sigma_{L}}{L}\right)^{2}+\left(\frac{\sigma_{P}}{P}\right)^{2}}
$$

where $L$ is the decay distance and $\mathrm{P}$ is the momentum of the $B$. To resolve the rapid $B$, oscillations, the proper time resolution must be on the order of the period of oscillation.

So $\operatorname{far} B_{s}$ oscillations have not been observed and the current lower limit on $\mathrm{x}_{s}$ is 22 . The most significant problem in $B_{S}$ mixing analyses is the effective tagging efficiency. In Run II, CDF expects to have sensitivity for a $5 \sigma$ observation for $x_{s}$ values of 59 to 74 [27]. The Standard Model expection for $B_{S}$ mixing is $22<\mathrm{x}_{S}<31$ [28].

\section{Part II}

\section{Experimental Apparatus}

\section{Accelerator}

This analysis is based on data from the Fermi National Accelerator Laboratory (FNAL), referred to in the scientific community as Fermilab. Fermilab is named after Italian particle physics pioneer, Enrico Fermi. The collection of accelerators and detectors which make up Fermilab are located about a one hour drive west of downtown Chicago, Illinois. 


\subsection{Overview of Experiment}

The accelerators at Fermilab consists of a series of linear and circular accelerators which serve to increase the proton and anti-proton energy. An overhead schematic of the accelerator complex is shown in Figure 7. The proton and anti-proton beams travel through accelerators of increasing energies before being passed to the largest accelerator, the Tevatron ${ }^{7}$. Here, the counter-rotating beams collide every $3.5 \mu \mathrm{s}$ with a center of mass energy $(\sqrt{s})$ equal to 1.8 $\mathrm{TeV}$. The interactions occur at 6 regions around the Tevatron. Particle detectors sit at two of these: $\mathrm{B} 0$ and $\mathrm{D} 0$. The data in this analysis was collected with the CDF detector during Run I (May 1992 to February 1995). In the following sections, the particle acceleration process and the specialized detector components are described in detail.

\subsection{Acceleration of Particles}

Protons begin their journey to the Tevatron as gaseous hydrogen. Electrons are added through an electric discharge to form hydrogen ions and the gas is accelerated to $750 \mathrm{keV}$ by a Cockroft-Walton Chamber. A linear accelerator further accelerates the hydrogen atoms to $400 \mathrm{MeV}$ during which the ions pass through a carbon foil stripping them of their electrons and leaving protons. The booster, an alternating gradient accelerator (synchrotron) with a 150 meter diameter, accelerates the proton beam to $8 \mathrm{GeV}$. While in the Booster, the protons are divided into 12 bunches with approximately $2 \times 10^{11}$ protons per bunch. The bunches are delivered to the Main Ring, a larger synchrotron with a diameter of $2 \mathrm{~km}$. Here

\footnotetext{
${ }^{7} 6.28 \mathrm{~km}$ circumference.
} 
the proton beam is accelerated to $150 \mathrm{GeV}$. At this stage, some percentage of the protons are injected into the Tevatron, others are used to produce anti-protons, and the remaining protons are sent to fixed target experiments via the linear accelerator.

The protons that are designated to make anti-protons are collided with a fixed tungsten or nickel target. Through a process which is essentially proton-nucleon scattering, the anti-protons are produced in addition to other final state particles. Since the anti-protons are produced with a spread of energy, they are sent to the Debunching Ring [29]. The Debunching Ring decreases the variance in momentum by adiabatic debunching and "RF bunch rotation" and reduces the transverse profile of the beam by stochastic cooling. This reduces the longitudinal and transverse oscillations. The anti-proton beams are concentrated before stacking into the accumulator. The cooled anti-protons are stored in the accumulator and are grouped into bunches consisting of approximately $6 \times 10^{11}$ anti-protons per bunch. The anti-protons are then accelerated to $150 \mathrm{GeV}$ in the Main Ring and are then delivered to the Tevatron in the opposite (counter-clockwise) direction of the protons.

\subsection{Particle Collisions}

The protons and anti-protons are grouped into six separate bunches each orbiting the Tevatron in opposite directions. Each bunch is spread approximately $30 \mathrm{~cm}$ along the beam direction. The bunches are accelerated to $900 \mathrm{GeV}$ inside a $5.7 \mathrm{~T}$ magnetic field of superconducting dipole magnets. The beams are focused to a transverse diameter (perpendicular to the beam direction) of $25 \mu \mathrm{m}^{2}$. The center-of-mass energy of the collision is $1.8 \mathrm{TeV}$.

The rate of collisions, $\mathrm{r}$, is the product of the physics cross section, $\sigma_{p}$, and the instanta- 
neous luminosity, $\mathcal{L}$ :

$$
r=\sigma_{p} \mathcal{L}
$$

The instantaneous luminosity is defined as a product of the number of protons and antiprotons, $N_{p}$ and $N_{p}$ respectively, the number of bunches, $N_{\text {bunches, }}$, and the bunch revolution frequency, $f$, divided by the the geometric cross sectional area of the beam, $\sigma_{A}$ :

$$
\mathcal{L}=\frac{N_{\mathrm{p}} \mathrm{N}_{\mathrm{p}} \mathrm{N}_{\text {bunches }} f}{\sigma_{\mathrm{A}}}
$$

The revolution frequency is approximately $50 \mathrm{kHz}$. The instantaneous luminosity is highest at the beginning and steadily declines as the anti-protons are lost to collisions and other environmental factors such as beam instability. The average instantaneous luminosity for Run $1 \mathrm{~b}$ equaled $1.6 \times 10^{31} \mathrm{~cm}^{-2} \mathrm{~s}^{-1}$.

The data collected during Run I is divided into two parts. Run $1 \mathrm{~A}$ defines the period between August 1992 through June 1993. The integrated luminosity was equal to 19.3 $\mathrm{pb}^{-1}$. Run $1 \mathrm{~B}$ defines the period from December 1993 through July 1995. The integrated luminosity was equal to $90.0 \mathrm{pb}^{-1}$. The total integrated luminosity for the data collected from Run $1 A$ to Run $1 \mathrm{~B}$ totaled $109.4 \pm 7.2 \mathrm{pb}^{-1}$. The highest instantaneous luminosity was $2.5 \times 10^{31} \mathrm{~cm}^{-2} \mathrm{~s}^{-1}$. 


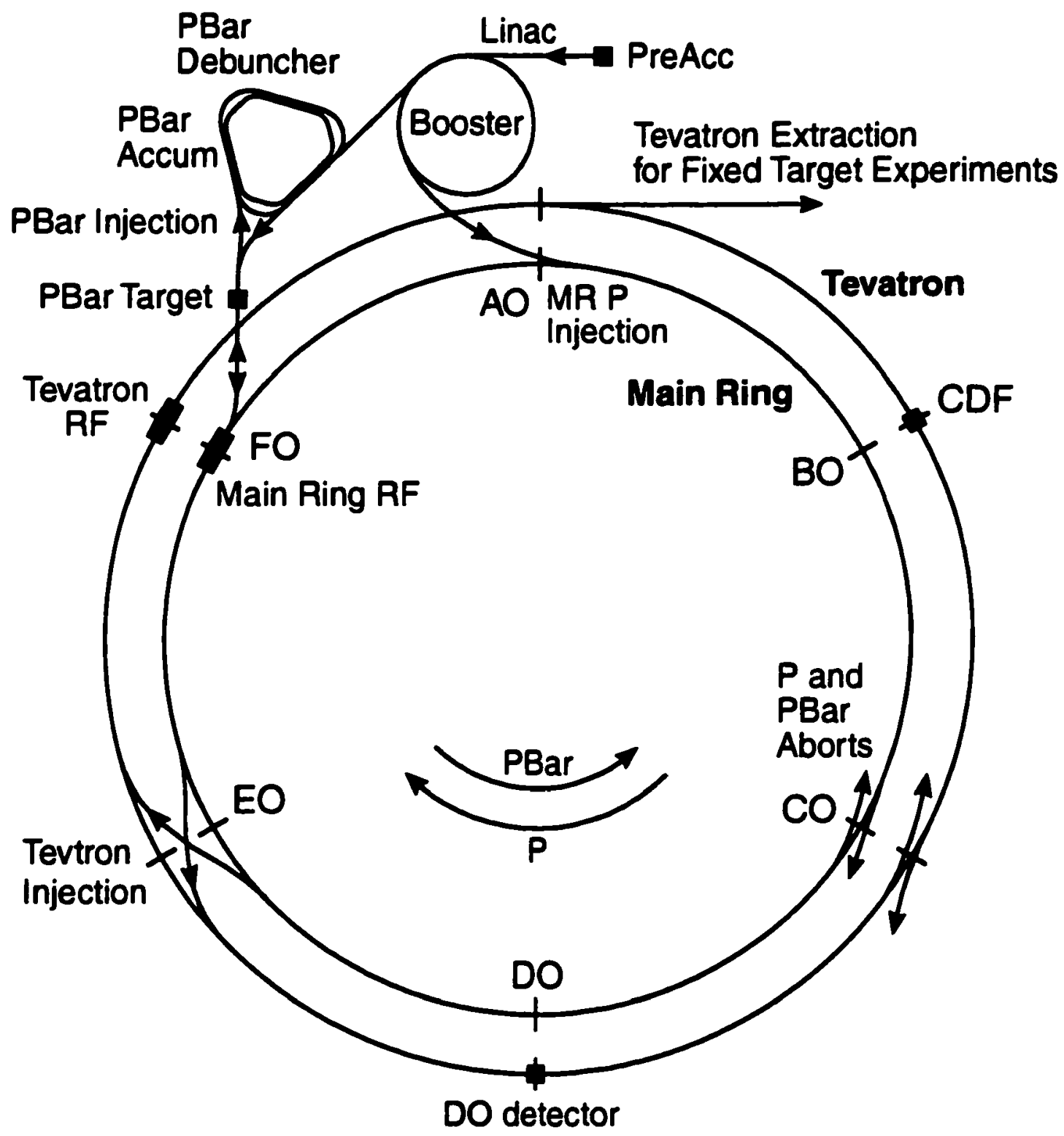

Figure 7: Diagram of the Fermilab $p \bar{p}$ accelerators. 


\section{CDF Detector}

In this section the CDF detector is explained in detail. First the measured variables and coordinate system are introduced in Sections 6.1 and 6.2. In Section 6.3 the specialized detector subsystems are described. The CDF trigger system is outlined in Section 6.4.

\subsection{Coordinate System}

The CDF detector has a cylindrically symmetric layout which surrounds the beam pipe at the interaction region. Measurements made by the detector are based on a cylindrical coordinate system orientated with respect to the beam axis. Since the proton and anti-proton beams are unpolarized, the physical process resulting from their interaction are invariant under rotations about the beam axes.

The positive z-axis is arbitrarily chosen to point along the direction of the proton. The polar angle is measured from the direction of the proton beam and the azimuthial angle $\phi$ from the horizontal plane. The angle $\theta$ is thus referenced to the positive $z$-axis and angle $\phi$ is measured with respect to the positive $x$-axis.

Ideally, the center of the beam aligns exactly with the center of the detector, however, the beam alignment is not perfect. The beam position changes from store to store. The affect of this is a shift of approximately 400 to $1000 \mu \mathrm{m}$ in the y-direction and a 200 to 1200 $\mu \mathrm{m}$ shift in the $\mathrm{x}$-direction. In addition, the beam and the detector $z$-axis are not exactly parallel. 


\subsection{Variables}

The difference in the interacting parton momentum affects the choice of variables which are the most useful to measure. As previously mentioned, partons inside the proton and antiproton carry an unkown percentage of the proton and anti-proton momentum. This fraction is described by structure functions. The consequence of this inbalance $\left(\Sigma P_{z} \neq 0\right)$ is a boost of the final state particles along the z-axis. Obviously, this boost differs from event to event. Therefore, variables which are invariant to boost are the best to measure. These include quantities transverse to the beam-line such as the transverse momentum, $P_{T}$. Quantities that are only measured in the plane transverse to incoming parton direction are denoted by a ' $T$ '. For example:

$$
P_{T}=P \sin \theta
$$

The rapidity of a track is defined as:

$$
y=\frac{1}{2} \ln \left(\frac{\mathrm{E}+\mathrm{P}_{\mathrm{z}}}{\mathrm{E}-\mathrm{P}_{\mathrm{z}}}\right)
$$

Rapidities are additive for boosts. In addition, psuedorapidity, defined as:

$$
\eta=-\ln [\tan (\theta / 2)]
$$

is frequently used in place of the polar angle since for highly relativistic particles $y \approx \eta$.

Charged particles are detected as tracks. A track is defined by five variables which define the helical path of a charged particle in a constant magnetic, $\vec{B}$, field. CDF uses the following properties to define a track [30]:

- The cotangent of the polar angle $(\cot \theta)$, 
- The curvature $(\mathrm{C})$ or transverse momentum $\left(P_{T}\right)$,

- The z-position at the point of minimum approach to origin $\left(z_{0}\right)$,

- The azimuthial angle of the track at minimum approach $\left(\phi_{0}\right)$,

- The impact parameter of the helix with respect to the beam line $\left(d_{0}\right)$.

\subsection{Detector Components}

The CDF detector is divided into three sections: the central $(|\eta|<1.1)$, the end plug region $(1.1<|\eta|<2.4)$ and the forward $(2.2<|\eta|<4.2)$. For this analysis, only the central portion of the CDF detector is used. From the interaction point outwards, there are four tracking chambers and a calorimeter. The innermost detector is a solid-state tracking device called the Silicon Vertex Detector (SVX). Surrounding the SVX is the Vertex Time Projection Chamber (VTX). The VTX provides tracking in the $r-\eta$ directions, the location of the primary vertex along the $z$-axis and identifies multiple intereactions in the same beam crossing. A cross sectional view of the detector is shown in Figure 8.

The Central Tracking Chamber (CTC) surrounds the VTX. The CTC provides the primary tracking information. Specifically, it measures the three-dimensional momentum and position of the track, which is required for exclusive $B$ hadron reconstruction. All of the these detectors sit inside a $1.4 \mathrm{~T}$ solenoidal magnetic field orientated along the proton beam direction. Outside the solenoidal magnet are the Central Electromagnetic (CEM) and Hadronic Calorimeters (CHA). The CEM measures energy deposited in its towers, which can be matched with tracks and used to identify electrons. Inside the CEM is the Central Strip 
Chamber (CES). The shape of the electromagnetic shower is characteristic of the incident charged particle. A layer of steel surrounds the calorimetery and prevents all tracks except muons and neutrinos from passing through. Outside the steel are two muon chambers: the Central Muon Chamber (CMU) and the Central Muon Upgrade (CMP).

The luminosity is measured by beam-beam counters. These consist of two plates of scintillator counters positioned on the front of each of the forward calorimeters. The details of the detector components which are specialized for measuring certain particle properties starting from the beamline moving outwards are presented in the following sections.

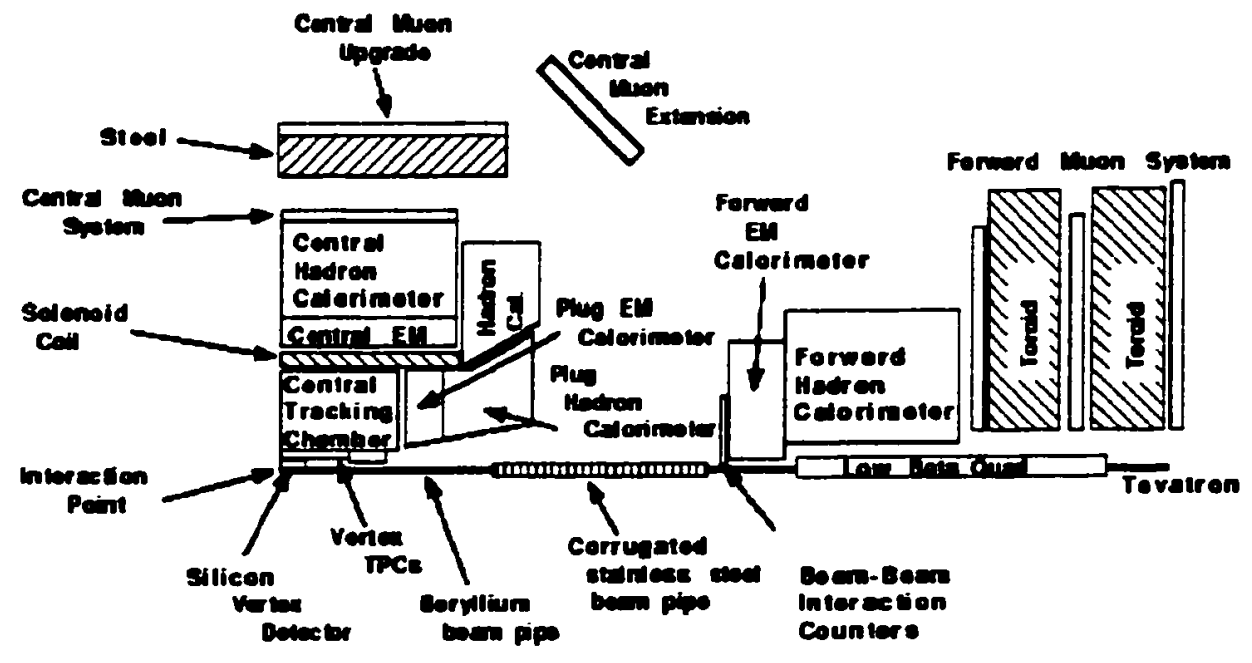

Figure 8: A cross sectional view of a quarter of the CDF detector. 


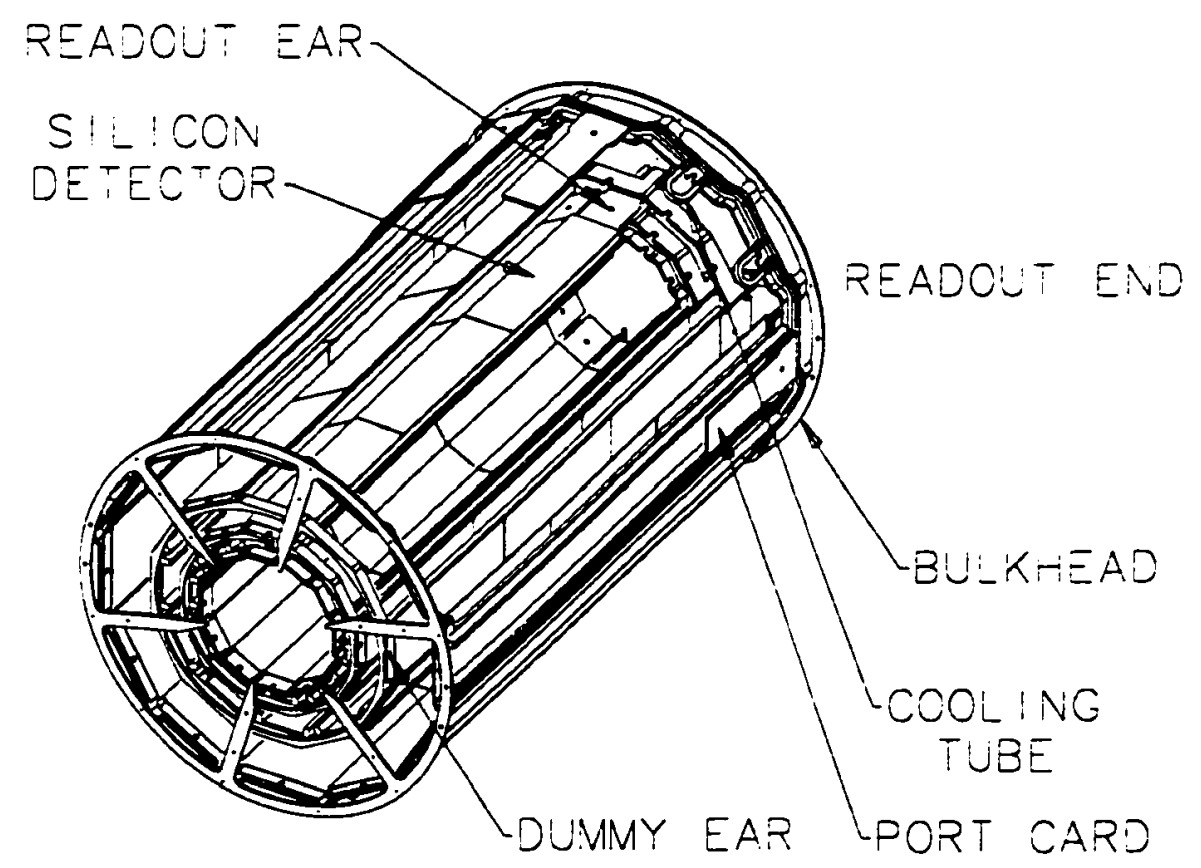

Figure 9: A 3D view of the SVX detector. 


\subsubsection{Silicon Vertex Detector}

The SVX is a silicon microstrip vertex detector [31]. A three-dimensional view of the SVX is shown in Figure 9. The SVX provides high precision measurements in the transvere plane close to the beam line. The SVX was designed to allow extrapolation of the track's helical path inside the beampipe. This allows the identification of secondary vertices of non-prompt tracks. The identification of these vertices is essential for $B$ physics measurements.

The detector is enclosed in a $51 \mathrm{~cm}$ long cylinder, that is separated into two barrels on each side of $z=0$. The SVX consists of four concentric layers of silicon detectors surrounding the $1.9 \mathrm{~cm}$ radius beryllium beampipe. The four layers are at radii: $3.0,4.2,5.7$, and $7.9 \mathrm{~cm}$. Each layer has 12 axial 'ladders' that subtend 30 degrees in azimuth. Six $8.5 \mathrm{~cm}$ long silicon sensors are mounted on each ladder. A charged particle passing through the silicon ionizes the silicon atoms causing the resulting drift electrons to be picked up by copper strips on the surface of the silicon. The strips run parallel to the beam and thus provide $\phi$ information. This results in a $13 \mu \mathrm{m}$ transverse spatial resolution and an impact parameter resolution, $\sigma_{d_{0}}$, equal to $17 \mu \mathrm{m}$ for the SVX. Because the $p \bar{p}$ interaction region is $60 \mathrm{~cm}$ long, the geometric acceptance of the SVX is $60 \%$.

For Run 1B, the SVX was replaced by the SVX'. The SVX' has lower noise and is more radiation hard. In addition, the inner radius is $2.9 \mathrm{~cm}$ (instead of $3.0 \mathrm{~cm}$ ). The $\mathrm{SVX}^{\prime}$ offers an improved spatial resolution of $11.6 \mu \mathrm{m}$ and an improved impact parameter resolution of $15 \mu \mathrm{m}$. 


\subsubsection{Vertex Time Projection Chamber}

The VTX surrounds the SVX $\left(\mathrm{SVX}^{\prime}\right)$ and has an outer radius of $22 \mathrm{~cm}$. A schematic of the VTX is shown in Figure 10. The detector volume extends $132 \mathrm{~cm}$ from $z=0$ in each direction and contains 28 drift modules azimuthally segmented into octants. Each module contains 2 drift regions separated by an aluminum high voltage grid to minimize drift time. The electric field is $1.6 \mathrm{KV} / \mathrm{cm}$ and the gas mixture is $50: 50$ argon and ethane. Each octant contains 16 or 24 sense wires depending on the $z$ position. The VTX measures charged particle trajectories in the $\mathrm{r}-\mathrm{z}$ plane for $|\eta|<3$. The $\mathrm{z}$ position of a track with respect to a wire in a given module is determined by drift time. The radius information is determined from the radial location of the wire. The average position resolution is $2 \mathrm{~mm}$ but depends on the number of detected tracks and primary interactions.
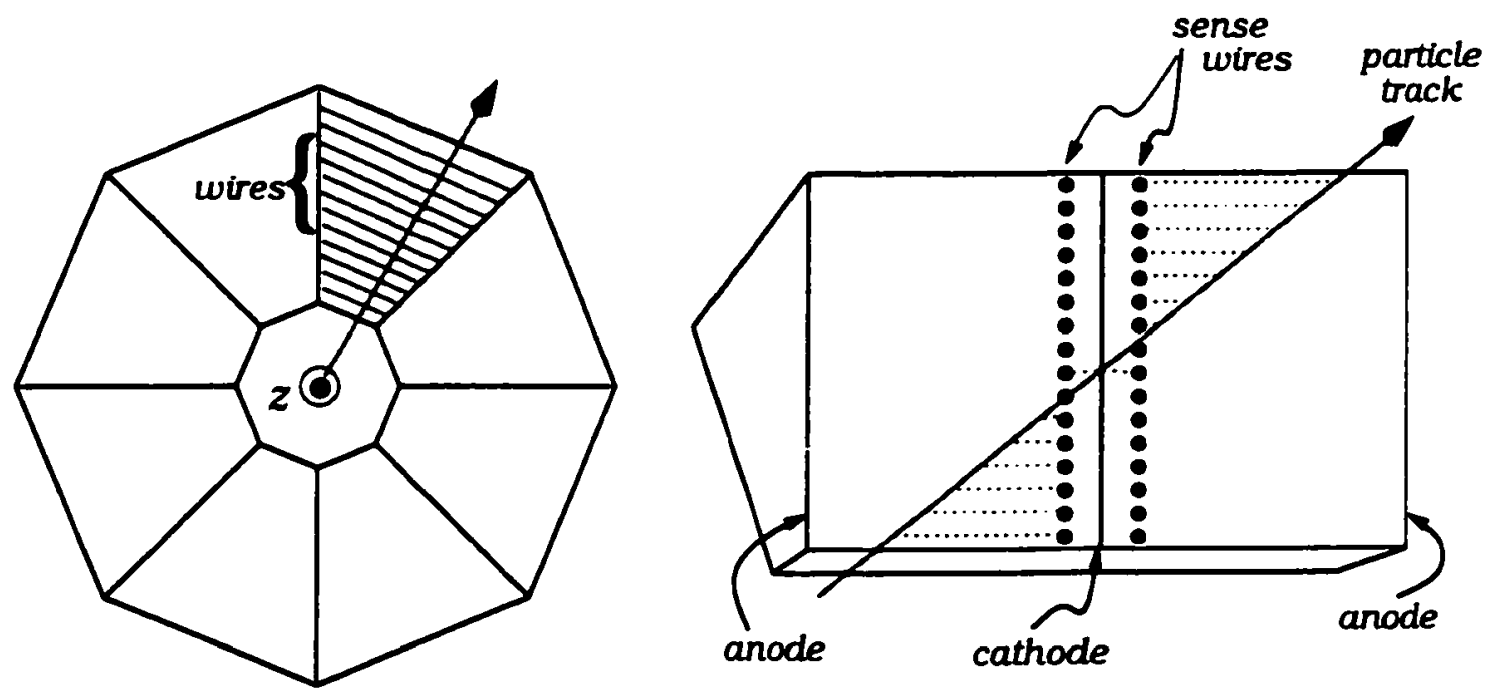

Figure 10: A schematic of the VTX detector. 


\subsubsection{Central Tracking Chamber}

The CTC surrounds the VTX and has an outer radius of 1.38 meters and extends 1.6 meters from $\mathrm{z}=0$ in both directions with pseudorapidity range $|\eta|<1.0[32]$. The cylindrical volume is filled with an argon, ethane, and alcohol mixture that is subjected to an electromagnetic field provided by wires strung through the chamber. There are 84 layers of $40 \mu \mathrm{m}$ diameter gold-plated tungsten sense wires arranged into nine superlayers. Five of the superlayers contain 12 layers of axial (parallel to the beam) sense wires. Four of the superlayers contain six layers of stereo sense wires at $\pm 3 \%$ relative to beam. The stereo superlayers lie between each axial superlayer. The superlayers are divided into drift cells such that the maximum drift distance in any cell is less than $40 \mathrm{~mm}$. An end view of the CTC is shown in Figure 11 .

A charged particle ionizes the gas molecules and the liberated electrons travel toward sense wires. The drift time of these electrons is used to measure spatial position. This causes the electrons to drift in a direction perpendicular to the radial direction. The large number of sense wires provides the ability to reconstruct the helical path of the tracks. Stereo layer information, when combined with axial layers, gives a measure of $z_{0}$ and $\cot \left(\theta_{0}\right)$ of tracks.

The $P_{T}$ of charged particles is calculated by measuring the track curvature, $R$, inside the 1.4 Tesla magnetic field, $B$,:

$$
\frac{1}{2 R}=\frac{c B}{2 P_{T}} \times 10^{-19}
$$

As the momentum increases, the curvature of the tracks becomes more uncertain as the arc 
approximates a straight line. The momentum resolution of CTC is:

$$
\frac{\delta P_{T}}{P_{T}^{2}}=0.002(\mathrm{GeV} / \mathrm{c})^{-1}
$$

In addition, the tracks are projected into SVX to search for matching SVX clusters. If 3 or 4 SVX layers have matching clusters a combined fit of CTC and SVX information is performed. The tranverse momentum resolution of SVX and CTC is:

$$
\frac{\delta P_{T}}{P_{T}}=\sqrt{\left[\left(0.0009 P_{T}\right)^{2}+(0.0066)^{2}\right]}
$$

\subsubsection{Central Electromagnetic Calorimeter}

The CEM sits just outside of the solenoid and inside the hadronic calorimeter [33]. It consists of 48 wedge shaped modules, each subtending 15 degrees in $\phi$. Each $2.5 \mathrm{~cm}$ long wedge is divided into 10 projective towers of width 0.1 in pseudorapidity. These 480 towers are orientated radially and point towards the interaction region The $\eta-\phi$ projection of tower is $\Delta \eta=0.1, \Delta \phi=15$ degrees. Each CE.M modules is constructed of 30 layers of $3.2 \mathrm{~m}$ lead sheets clad in $0.38 \mathrm{~mm}$ aluminum plating between 31 layers of $5 \mathrm{~mm}$ thick polystrene scintillator clad in $0.38 \mathrm{~mm}$ vellum drawing paper. The layers rest on an aluminum base plate.

The radiation length of material is uniform for all azimuthial angles as the amount of lead in specific towers varies as a function of $\eta$. The total depth of $34.5 \mathrm{~cm}$ is approximately 18 radiation lengths (approximately one hadronic interaction length). The CEM is designed to measure the ioinization shower produced by electrons. The shower is produced by the 
interaction of the charged particle with the lead. The electrons and positrons excite molecules in the scintillator which emit photons. Wavelength shifters on the sides of each towers collect the scintillator light and propogate it to photomultiplier tubes via light guides. The inital electron energy can be calculated based on the total light collected. The average energy resolution is:

$$
\frac{\sigma(E)}{E}=\frac{13.7 \%}{\sqrt{E_{T}}} \ominus 2 \%
$$

\subsubsection{Central Strip Chamber}

The CES provides the position and transverse development of electromagnetic showers near shower maximum in the CEM. It sits between the eighth and ninth scintillator layer of the CEM. At a depth of approximately 5.9 radiation lengths, the CES transverse profile of the shower is measured with a gas chamber with sense wires transverse to the radial direction. It consists of proportional wire chambers with orthogonal cathode strips and anode wires. Gold plated tungsten wires in the center of each cell run the length of the chamber and are cleaved in the center of the chamber providing independent readout at both ends. Electron showers profiles were measured during test beam runs. The CES has a position resolution of $2 \mathrm{~mm}$ for $50 \mathrm{GeV} / c$ electrons with the resolution degrading for lower energy electrons.

\subsubsection{Central Hadronic Calorimeter}

Each tower in CEM is matched in the CHA. The CHA is constructed of 32 layers of $2.5 \mathrm{~cm}$ thick steal absorber alternating with $1.0 \mathrm{~cm}$ thick plastic scintillator [34]. 


\subsubsection{Muon Detectors}

The muon chambers are located behind the CHA. Only muons and neutrinos are likely to reach the muon chambers. Wuons are heavier then electrons and thus do not lose as much energy to bremsstrahlung. The central muon system, CMU, is located at 5.4 pion interaction lengths. The central muon upgrade, C.MU, is outside the return magnetic yoke at 8.4 pion interaction lengths. A schematic of a CMC module is shown in Figure 12. Both the CMC and CMP are made of four layers of rectangular drift chambers which measure track stubs that are then matched to tracks in the CTC. Sense wires are parallel to the beam in the center of each cell. If a track appears to point from CTC to a stub it is a likely match. The $\mathrm{r}-\phi$ location of the sense wire is offset by $2 \mathrm{~mm}$ for alternate layers. The CMU has $|\eta|<0.6$ and $85 \%$ coverage in $\phi$. Beyond the CMC, there is an additional $60 \mathrm{~cm}$ of steel, followed by the central muon upgrade chamber (CMP). The CMP covers $|\eta|<0.6$ with $65 \%$ coverage in $\phi$. The Central Muon Extension, C.MX, extends the coverage in $\eta$.

\subsection{Trigger}

The interaction rate is so high that it is not possible to record every $p \bar{p}$ event. Events occur every 3.5 microseconds which is equivalent to 300,000 collisions per second. Since interesting events occur less often then uninteresting ones, the CDF trigger was built to reduce the overall rate while being efficient for events of interest [35]. The trigger system records, at most, 500 events per second. To record events at a higher rate requires faster electronics and more data storage. 
The trigger has four stages ( 0 through 3 ). The first three levels are hardware based. Level 0 requires a proton anti-proton bunch crossing and thus eliminates cosmic ray events. On average $1.5 p \bar{p}$ collisions occur per beam crossing. The number of collisions is governed by Poisson statistics. There are two sets of scintillator plates, called Beam-Beam Counters (BBC), located $6 \mathrm{~m}$ on either side of $\mathrm{z}=0$ with $3.2<|\eta|<5.9$. Almost every interaction has particles in the fiducial area for beam counters.

The detector elements are connected to "front end" electronics. Level 1 requires a high $P_{T}$ electron or candidate in muon chamber or two neighboring calorimeter towers above threshold and thus low $P_{T}$ electron or muon candidate. The level 1 trigger has an input rate of $300 \mathrm{kHz}$ and outputs data at $1 \mathrm{kHZ}$. The level 2 trigger combines calorimeter and muon information with the tracking information. Level 2 outputs at $12 \mathrm{~Hz}$. Only tracks with $P_{T}>$ $7 \mathrm{GeV} / c$ are matched to electron or muon candidates. If the event passes level 2 , then whole detector is read out in approximately $3 \mathrm{~ms}$ and the data is passed to the level 3 trigger. The level 3 trigger is a software based trigger. Data can be recorded at 5 events per second ( 5 $\mathrm{Hz}$ ). The event builder collects data and sorts at rate of approximately $30 \mathrm{~Hz}$. The average size of an event is $200 \mathrm{kB}$. 


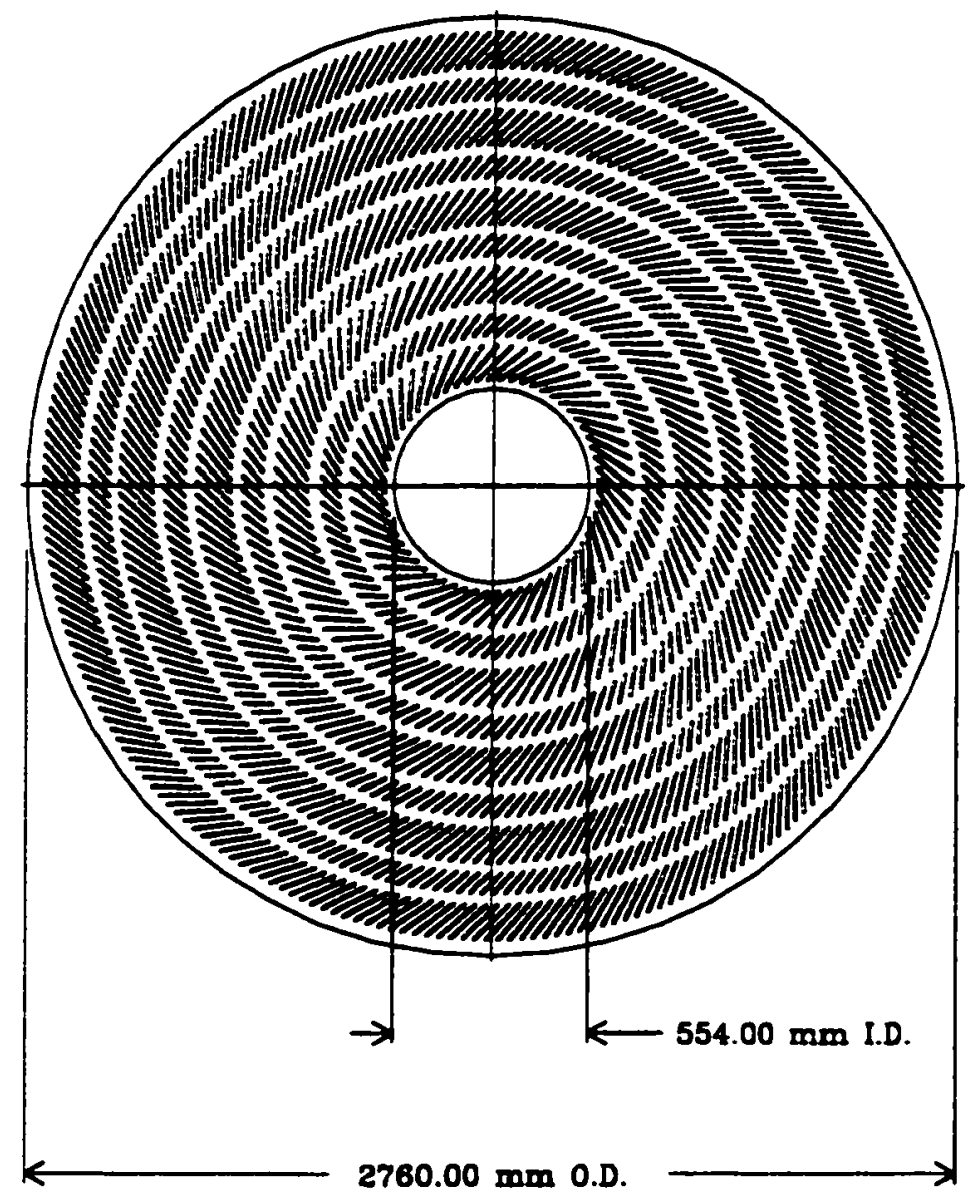

Figure 11: The Central Tracking Chamber endplate. 


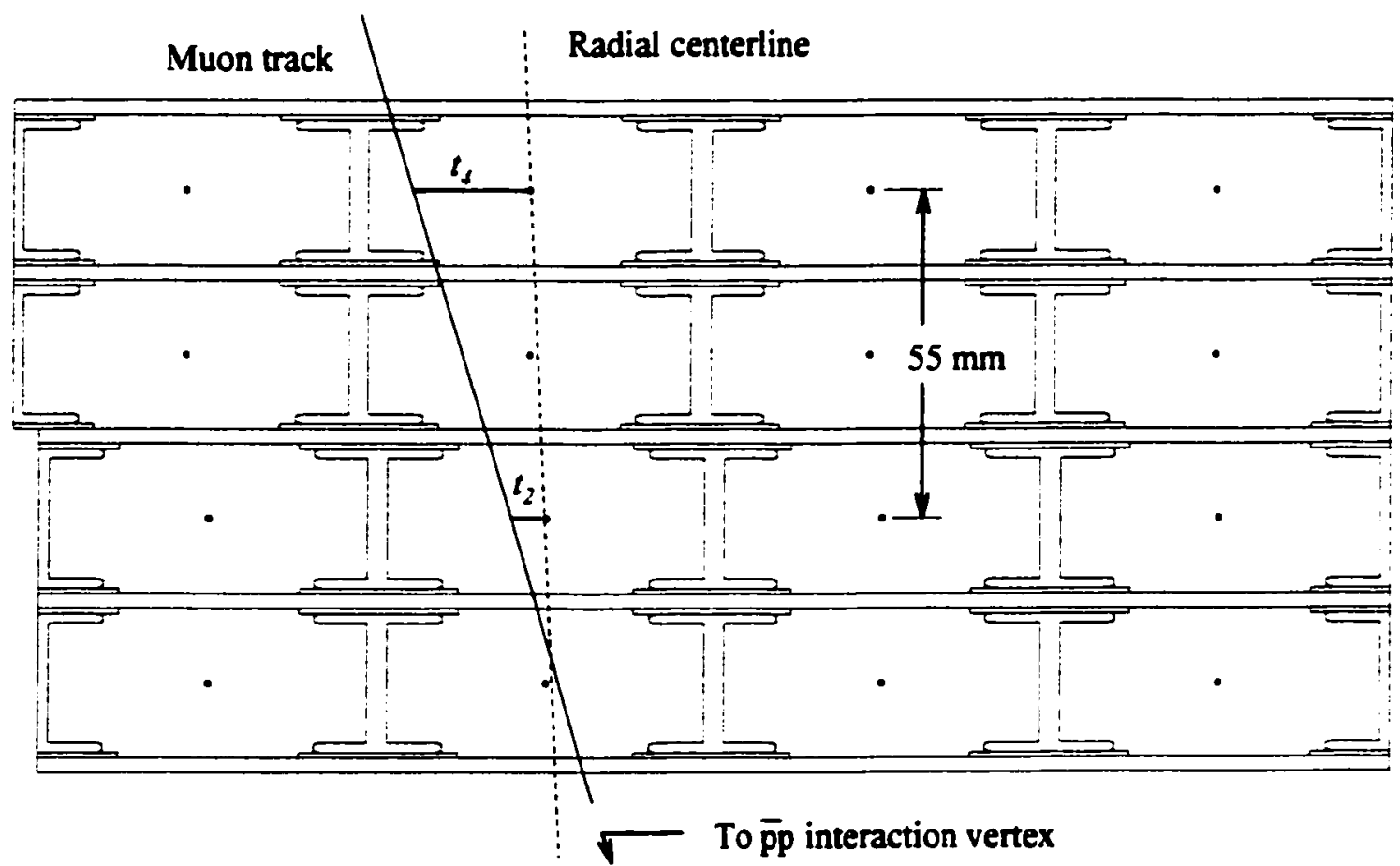

Figure 12: A schematic of the CMU modules. 


\section{Part III}

\section{Analysis}

The goals and procedures used in this analysis are described in Section 7 . In Section 8 the dataset used to reconstruct $B$ Mesons is described. The $B$ reconstruction process, including the basic selection requirements, optimization procedure, mass distribution of the $B$ candidates and a measurement of the $b \bar{b}$ fraction, are described in Section 9 and 10 . The $B_{s}$ search is detailed in Section 11.

\section{Goal}

The original goal of this analysis was a measurement the $B_{s}^{0} \rightarrow D_{s}^{-} \pi^{+}$decay rate. Based on the number of events reconstructed in the reference signal, it was noted that the actual observation a $B_{s}^{0} \rightarrow D_{s}^{-} \pi^{+}$signal was unlikely and the focus centered on placing the lowest possible upper limit on the $B_{s}^{0} \rightarrow D_{s}^{-} \pi^{+}$branching ratio. Calculating the branching ratio directly requires detailed information on the cross section $(\sigma(B))$, luminosity $(L)$, acceptance $(\alpha)$, and efficiencies $(\epsilon)$, as given by the following equation:

$$
N_{o b s}(f)=\int \mathcal{L} \mathrm{dt} \times \sigma(\mathrm{B}) \times \mathrm{BR}(\mathrm{B} \rightarrow \mathrm{f}) \times \epsilon \times \alpha .
$$

All of these factors must be measured separately and all systematic uncertainties on these factors (acceptance, selection efficiency and reconstruction efficiencies) will add into the total systematic error on the measurement. 


\subsection{Relative Measurement}

In the indirect method, a measurement of the branching ratio of the decay of interest is performed relative to a reference signal (a similar decay with known decay rate). In this method the $B$ production cross section, integrated luminosity, acceptance and their systematic uncertainties cancel. A simplified calculation of the branching ratio using the indirect method is:

$$
\frac{B R\left(B_{s}\right)}{B R\left(B_{\text {ref }}\right)}=\frac{\mathrm{N}_{\mathrm{B}_{\mathrm{g}}}}{\mathrm{N}_{\mathrm{B}_{\mathrm{ref}}}} \times \epsilon \times A
$$

where $\mathrm{N}_{B_{s}}$ and $\mathrm{N}_{B_{\mathrm{ref}}}$ are the number of $B_{s}$ and $B_{\text {ref }}$ mesons reconstructed, $\epsilon$ is the relative efficiency for $B_{s}$ and $B_{\text {ref }}$ reconstruction, $\mathrm{BR}\left(B_{s}\right)$ and $\mathrm{BR}\left(B_{\text {ref }}\right)$ are the respective branching ratios, and the factor $A$ is the ratio of fragmentation fractions.

\subsection{Reference Decay Modes}

The reconstruction of $B^{+}$and $B^{0}$ decays via their charmed hadronic decay products provides a reliable reference signal on which to base our limit on the $B_{s}^{0} \rightarrow D_{s}^{-} \pi^{+}$branching ratio, since the $B^{+} \rightarrow \overline{D^{0}} \pi^{+}, \overline{D^{0}} \rightarrow K^{+} \pi^{-}$and $B^{0} \rightarrow D^{-} \pi^{+}, D^{-} \rightarrow K^{+} \pi^{-} \pi^{-}$product branching ratios are well known. In addition, the similar decay kinematics of these decays will eliminate many systematic uncertainties which would exist in a direct measurement. The product branching ratio of the $B^{+}$and $B^{0}$ decay modes studied are [1]:

- $\mathrm{BR}\left(B^{+} \rightarrow \overline{D^{0}} \pi^{+}, \overline{D^{0}} \rightarrow K^{+} \pi^{-}\right)=(0.027 \pm 0.004) \times 10^{-2}$,

- $\mathrm{BR}\left(B^{0} \rightarrow D^{-} \pi^{+}, D^{-} \rightarrow K^{+} \pi^{-} \pi^{-}\right)=(0.020 \pm 0.002) \times 10^{-2}$. 


\section{DataSet}

The inclusive lepton dataset, which should contain a large number of $B$ mesons is used. However, a large portion of the events in this sample do not contain the decays of interest. To identify the events of interest, the topology of the decays and the copious background events must be understood. To reconstruct and identify $B$ mesons in the presence of a copious background, the events selection cuts must be chosen to reduce the number of fake $B$ 's while retaining the real $B$ 's. The datasets and trigger criteria are described in Section 8.1. The Monte Carlo dataset used to model the real $B$ events and guide the selection cuts is detailed in Section 8.2.

\subsection{Inclusive Lepton Dataset}

Since the semileptonic branching fraction of $B$ mesons is relatively large, the inclusive lepton dataset should provide an ample source of $B$ 's. These decays occur through the electroweak process via the $b \rightarrow c$ transition. The $c$-quark from the decay fragments and forms a $D$ meson, which also in turn decays. The virtual $\mathrm{W}$ mediating the $b \rightarrow c$ transition, forms a quark anti-quark pair or a lepton and its neutrino. The event topology of interest occurs when the $\mathrm{W}$ mediating one $B$ hadron decay forms a lepton and its neutrino and a $W$ boson from the other $B$ meson forms a quark anti-quark pair (pion).

The inclusive lepton dataset consists of leptons which pass the single lepton trigger. At level 1 , the inclusive electron trigger requires the presence of single calorimeter tower with $\mathrm{E}_{T}>6-8 \mathrm{GeV} / c^{2}$. The inclusive muon trigger requires the presence of a track in the CMU 
and CMP. The level 2 electron and muon triggers require a charged track with $P_{T}>7.5$ $\mathrm{GeV} / c$ measured in the $\mathrm{r}-\phi$ plane of the CTC by a hardware track processor, the CFT. The electron trigger demands that the track match a cluster in the electromagnetic calorimeter with $E_{T}>8.0 \mathrm{GeV} / c^{2}$. The muon trigger demands the track match a segment in the CMU and CMP. The overall trigger efficiency turns on at $P_{T}=6 \mathrm{GeV} / c$, reaches $50 \%$ at $P_{T}=8$ $\mathrm{GeV} / c$, and reaches a $90 \%$ plateau at $P_{T}=10 \mathrm{GeV} / c$. At Level 3, more stringent electron and muon quality cuts are applied. Approximately $7.5 \times 10^{6}$ electron events and $2.5 \times 10^{6}$ muons were recorded in this dateset.

The lepton with the highest transverse momentum in the event, most likely the trigger lepton, is used as the lepton that defines the hemisphere to look for the hadronic $B$ decay products. Selecting events with one or more leptons of moderate to high transverse momentum is an efficient way to separate $b \bar{b}$ or $c \bar{c}$ events from the background. The $c \bar{c}$ events will have kinematic features similar to both the $b \bar{b}$ events and background events. However, the $c \bar{c}$ events have stable daughters with smaller, on average, transverse momentum, impact parameter, and proper decay length compared to $b \bar{b}$ events.

Events with at least one electron or muon passing a single-lepton trigger are selected. In addition, the leptons are required to be in the central detector and have $P_{T}^{\ell}>8.0 \mathrm{GeV} / c$. This purifies the sample by eliminating events which passed the trigger but did not have a real lepton. In the following sections the electron and muon identification criteria are described. 


\subsubsection{Electron Dataset}

The basic definition of an electron candidate is a track which points to a cluster in the electromagnetic calorimeter. Additional kinematic requirements are made on the electron candidates. These criteria include the transverse momentum of the tracks and transverse energy deposited in the calorimeter. In addition, the transverse profile of the electromagnetic showers are required to be consistent with known electron shower profiles based on test-beam measurements. Since the electron is not involved in the $B$ reconstruction, no requirement is made on the electron to be within the SVX tracking volume. A summary of the specific electron quality cuts are:

- No SVX requirement,

- Eliminate conversions,

- $P_{T_{l}} \geq 8.0 \mathrm{GeV} / c$,

- Ratio of the hadronic to electromagnetic energy, Had $/ \mathrm{Em},<0.04$,

- Distance between the strip-chamber shower position and the extrapolated track position, $\Delta x(C E S),<1.5 \mathrm{~cm}$

- Distance between the strip-chamber shower position and the extrapolated track position, $\Delta z(C E S),<3.0 \mathrm{~cm}$,

- Strip chamber cluster shape consistent with an electron shower, $\chi_{\text {strip }}^{2},<10.0$ and $\chi_{w i r e}^{2},<10.0$,

- Lateral shape of the shower, $L s h r,<0.2$. 


\subsubsection{Conversion Electrons}

In addition to the semileptonic decay of $B$ mesons, electrons are also produced through photon conversions, $\gamma \rightarrow e^{+} e^{-}$. To determine if an electron is the result of a photon conversion, a search for a conversion partner for each trigger electron is done. Conversion pairs originate from a common vertex with very small opening angle between them (since the parent particle, a photon, is massless). All tracks in the events are combined with the trigger electron to determine if they form a conversion. The following criteria are used to eliminate conversion candidates:

- $\mathrm{r}-\phi$ separation at tangent point $<0.5 \mathrm{~cm}$,

- $\Delta \operatorname{cotan}(\theta)<0.06$,

- $\mathrm{z}$ mismatch at tangent point $<5 \mathrm{~cm}$,

$--10 \mathrm{~cm}<$ radius of conversion $<50 \mathrm{~cm}$,

- Difference in azimuthial angles, $\Delta \phi,<0.05$,

- Pointing residual to origin $<1.0 \mathrm{~cm}$.

After the quality cuts, there are approximately four million electron events in the dataset.

\subsubsection{Muon Dataset}

The basic definition of a muon is a track which extrapolates into the muon chambers and is consistent with a muon stub. There are two single muon triggers. The first requires a 
CTC track with $P_{T}>7.5 \mathrm{GeV} / c$ that matches with muon stubs in both muon systems. The second requires a CTC track with $P_{T}>12 \mathrm{GeV} / c$ with a hit in any muon system with $|\eta|<1.0$. The criteria eliminate major sources of muon background due to charged kaons and pions decaying in flight to yield muons and hadronic particles entering or "ipunchingthrough" and entering the muon systems. The only kinematic constraints are a transverse momentum requirement and matching requirements. A summary of the muon selection cuts are:

- Muon stub in CMU, C.MP, CMX, CMU/CMP, CMU/CMX,

- Transverse momentum $P_{T}>8.0 \mathrm{GeV} / c$,

- CMU, CMP, CMX $x$ and $z$ matching $\chi^{2}<25$,

- $\operatorname{CMX} \Delta z<50$.

After the quality cuts, there are approximately $2 . \overline{5}$ million muon events in the dataset.

\subsection{Monte Carlo}

In this section, the simulation of heavy flavor creation and decay is described. To model the decays of interest, Monte Carlo events are generated. The Monte Carlo is supplemented with phenomenological models of hadronization and decays. These Monte Carlo events are used in the optimization procedure by providing a measurement of the reconstruction efficiencies for various selection criteria. PYTHIA simulates the entire $p \bar{p}$ collision according to pertubative 
QCD [36]. We use PYTHIA 5.7 to generate $b \bar{b}$ events. The fragmentation is described by models. The Monte Carlo input parameters are:

- $\operatorname{Mass}(b$-quark $)=4.75 \mathrm{GeV} / c^{2}$,

- $P_{T}(b$-quark $)>9.0 \mathrm{GeV} / C^{2}$,

- Rapidity, $\mid y(b$-quark $) \mid,<1.5 \mathrm{GeV} / c^{2}$,

- Peterson Fragmentation Parameter, $\epsilon,=0.006$,

- NDE generation with MRSD0 and $\mu=\mu_{0}$.

The events are processed by CLEOMC (also called QQ) which forces the $B$ mesons into the specific decay mode of interest [37]. These events are then filtered based on their decay and lepton quality cuts. The events are processed with a detector simulation program QFL'. This simulation creates hits in the SVX and peforms the complete track reconstruction in the SVX and in the CTC to simulate the detector response. The parameterized track resolutions are used. $\mathrm{QFL}$ ' also forces conversions and bremsstrahlung. QFL' models the reconstruction resolution of the various detector subsystems. The effect of $\mathrm{QFL}^{\prime}$ is a smearing of the generated tracks parameters. The output of $Q F L^{\prime}$ is in the same format as real data and is processed in the same manner from this point forward.

\section{$9 \quad B$ Meson Reconstruction}

This section describes the basic reconstruction process for all $B$ meson flavors. $B$ meson are reconstructed from their decay products since the $B$ does not live long enough, 1.6 
picoseconds, to be observed directly. To reconstruct a specific $B$ decay, basic quality cuts are applied to ensure that the track parameters are well measured, for example the tracks must be fully reconstructed inside the CTC volume. The generic decay topology is described in Section 9.1. The vertex identification, track quality cuts and $B$ meson reconstruction are described in Sections 9.2, 9.3, and 9.4, respectively.

\subsection{Decay Topology}

The basic decay topology of interest is $B$ meson decay opposite the semileptonic decay of the other $B$ meson produced in the event. A schematic of this topology is shown in Figure 13.

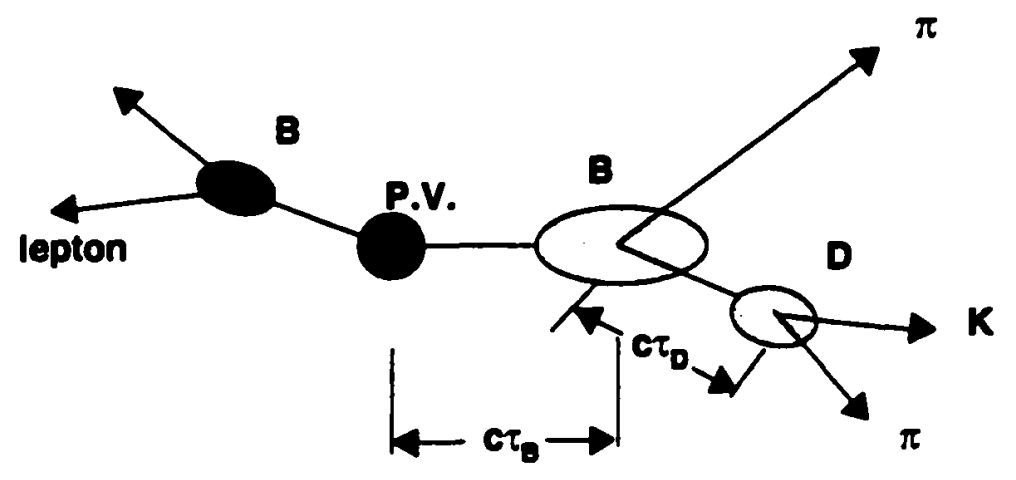

Figure 13: A schematic of the $b \bar{b}$ events of interest. One $B$ meson decays to $D \pi$ while the other $B$ decays semileptonically. 


\subsection{Primary Vertex}

The $z$-location of the primary vertex is measured by the VTX with a fixed uncertainty of 1 $\mathrm{mm}$. The $x-y$ coordinates of the primary vertex is determined by the tracks in the event with a resolution of $40 \mu \mathrm{m}$. During Run I, the $x-y$ beam position is measured for multiple events and a run average value is calculated. The vertex position varied by approximately $10 \mu \mathrm{m}$ during the run. The slope and intercept of the beam position when combined with the z-position for each event allows the measurement of the primary vertex location on an event-by-event basis. The impact parameter with respect to the primary vertex is measured for each of the daughter tracks. The ratio of the impact parameter and the uncertainty of the impact parameter error is used in this analysis to distinguish between prompt tracks coming from the primary vertex and displaced tracks coming from a displaced secondary vertex. Thus, tracks are required to have an impact parameter inconsistent with zero.

\subsection{Track Selection}

All tracks are required to have a three dimensional fit with four hits in the axial superlayers and two hits in the stereo superlayers. In addition, due to the need for precise vertex information, the SVX fit information is added to the CTC fit information for tracks with a mininimum of three SVX hits. If pions coming from a $K_{s}$ have less then three SVX hits, the CTC fit is used alone.

The tracks are required to have a $P_{T}>400 \mathrm{MeV} / c$ to ensure a high and reliable efficiency and a good resolution of the track parameters. Tracks with $P_{T}<250 \mathrm{MeV} / c$ do not exit 
through the outer cylinder of the CTC and spiral until they exit the volume through the endplates. Additionally, low momentum tracks are produced in large numbers, and their reconstruction is difficult due to the large number of intersecting helices. The pattern recognition algorithm therefore is tuned to only find tracks with $P_{T}>250 \mathrm{MeV} / \mathrm{c}$. However, high track-finding efficiency and good resolution of the track parameters is only achieved for tracks with $P_{T}>400 \mathrm{MeV} / c$. The impact parameter significance, the ratio of the impact parameter of the track, with respect to the primary vertex, to the uncertainty on the impact parameter, is required to be greater then 3 . This cut is intended to eliminate tracks obviously coming from the primary vertex. The kaons and pions are stable daughters and usually have large impact parameters with respect to the primary vertex. The beam spot $x-y$ resolution is $30 \mu \mathrm{m}$, thus the impact parameter with respect to the beam spot will also be large.

\subsection{Reconstruction}

The reconstruction of $D^{+}$and $\bar{D}^{0}$ mesons is a process of combining appropriately charged tracks. An oppositely charged pion and kaon are combined to make a $\bar{D}^{0}$ meson, and two oppositely charged pions are combined with a kaon to make a $D^{+}$meson. The difference in the $z$-position of these tracks is required to be less the $5 \mathrm{~cm}$. This is an adequately wide restriction considering that the $z$-resolution is approximately $1 \mathrm{~cm}$.

Pion-kaon combinations whose invariant mass is within $100 \mathrm{MeV} / \mathrm{c}^{2}$ of the respective nominal $D$ mass are constrained to originate from a common vertex. During this process, the tracking parameters are allowed to change within their errors to find the most probable solution with the common vertex constraint. This effectively changes the track parameters 
and thus changes their invariant mass and improves the mass resolution. The resulting fitted vertex is required to pass a maximal chi-squared of the fit, where the probability of the chisquared be greater than $0.1 \%$. This removes candidates which obviously do not come from the same vertex. In addition the invariant mass of the vertex pair is required to be within $20 \mathrm{MeV} / c^{2}$ of the nominal $D$ mass. The Monte Carlo mass distribution for $\bar{D}^{0}$ and $D^{+}$ candidates are shown in Figures 14 and 15. A final pion candidate is combined with the $D$ candidates tracks to form a $B$ candidate. The mass of the $B$ candidate must be within 1.0 GeV of the nominal $B$ mass. Tracks which pass this cut are vertexed together, with the invariant mass of the $D$ candidate constrained to the nominal $D$ mass. Additionally, the impact parameter of the $B$ candidate is required to be within $80 \mu \mathrm{m}$ of the primary vertex. Events with a $B$ candidate mass between $4.5<\mathrm{M}_{\mathrm{B}}<6.0 \mathrm{GeV} / \mathrm{c}^{2}$ are accepted. The Monte Carlo mass distributions for $B^{+}$and $B^{0}$ candidates are shown in Figures 16 and 17.

\subsection{Summary of Reconstruction Cuts}

A summary of all the basic reconstruction cuts performed before the optimization cuts are:

- $1.8445<m\left(\vec{D}^{0}\right)<1.8845 \mathrm{GeV} / c^{2}$,

- $1.8493<m\left(D^{+}\right)<1.8893 \mathrm{GeV} / c^{2}$,

- Probability of the vertex fit $>0.1 \%$ for $D$ and $B$,

- $P_{T}\left(\pi_{D}\right)>0.4 \mathrm{GeV} / c$

- $P_{T}\left(\pi_{B}\right), P_{T}\left(K_{D}\right)>0.6 \mathrm{GeV} / c$ 
- $|\Delta z|<5 \mathrm{~cm}$ for all final state tracks,

- The number of SVX hits for all tracks $\geq 3$,

- Impact parameter significance for all tracks, $\left|\frac{d_{0}}{\sigma_{d_{0}}}\right|,>3$,

- Isolation ${ }^{8}>0.65$,

- Impact parameter of $B$ candidate $<80 \mu \mathrm{m}$.

\section{Signal Optimization}

This section describes the final selection of the $B$ candidates. Most of the reconstructed events which pass the basic selection cuts are combinatorics (random combinations of tracks) which happen to have a mass in the required mass region. For example, in the reconstruction of $B^{+} \rightarrow \overline{D^{0}} \pi^{+}, \overline{D^{0}} \rightarrow K^{+} \pi^{-}$events, all $b \bar{b}$ and $c \bar{c}$ events which produce a $D \rightarrow K \pi$ have potential to produce a $B^{+}$candidate. For instance, a track from the primary vertex may form a good vertex with the $D$ and fake a $B$ signature.

To filter out the real $B$ events, additional selection criteria are introduced. Multiple cut thresholds for each criteria are scanned to select the optimal value. Some of the sources contributing to the background, the variables used to reduce this background, and the optimization procedure are presented in the following sections.

\footnotetext{
${ }^{8}$ Described in Section 10.2 .3
} 


\subsection{Sources of Background}

There are essentially three types of backgrounds present in the reconstructed sample. There are $B$ candidates formed from random combinations of tracks in $b \bar{b}, c \bar{c}$, and QCD-events, track combinations in actual $b \vec{b}$-events made from fake $D$ candidates and track combinations in $b \bar{b}$ and $c \bar{c}$-events made from real $D$ candidates.

Fake $B$ candidates can come from many different souces. Occasionally, the random combination of tracks from QCD production of $u, d, s$-quarks can form $B$ candidates. This is usually referred to as combinatoric background. In these events, the track parameters may be so poorly measured, for instance a track formed from hits of more then one real track, that $B$ candidates are formed that appear to have the $B$ 's characteristic lifetime. Secondary vertices and detached tracks can also be the products of misreconstructed tracks, multiple intereactions per beam crossing, fake vertices, and strange particles. Thus, tracks with impact parameters which are too large must be removed. Strange particles have long lifetimes but are produced $10^{2} \rightarrow 10^{3}$ more readily then $B$ hadrons. Other sources arise when a real $D$ candidate is combined with a non- $B$ track to produce a fake $B$ candidate.

\subsection{Background Reduction}

To reduce the number of background events, variables whose distributions allow the efficient removal of fake events are selected. The choice of variables is guided by the Monte Carlo simulation of the particle decay of interest. For each variable the Monte Carlo distribution is compared with the distribution from the data candidates outside the $B$ signal region. The 
variables used are presented in the following sections.

\subsubsection{Transverse Momentum}

The transverse momentum of real $B$ and $D$ mesons will be, on average, higher then the fake $B$ and $D$ candidates. Since the fake events constitute the majority of the background events, a minimum transverse momentum requirement is expected it to significantly reduce the background. A range of minimum $P_{T}$ values from $6 \mathrm{GeV} / c^{2}$ to $15 \mathrm{GeV} / c^{2}$ are scanned.

\subsubsection{Proper Decay Time}

Although there are numerous background processes with large cross sections, they do not all produce particles with large proper decay time. $A B$ or $D$ meson has a long lifetime allowing for a displaced secondary vertex. Due to relativistic kinematics, it travels far enough away from the primary vertex such that its decay products can be discerned from those tracks coming from the primary vertex. The proper decay time, $c \tau$, is defined as $c$ times the proper decay time and is given by:

$$
c \tau=\mathrm{L}_{x y} \frac{\mathrm{M}}{\mathrm{P}_{\mathrm{T}}}
$$

where the transverse decay length $L_{x y}$ is the projection, in the xy-plane, of the decay length vector on the transverse momentum:

$$
L_{x y}=\frac{\vec{\ell}_{x y} \cdot \vec{P}_{T}}{P_{T}}
$$

The $\vec{\ell}_{x y}$ vector points from primary vertex to the $B$-vertex or from the $B$-vertex to the $D$ vertex. The Monte Carlo proper decay time of $B$ and $D$ mesons is shown in Figure 18. The 
cuts on the proper decay time do not efficiently reduce the background events from other $B$ and $D$ meson decays.

\subsubsection{Isolation}

An additional variable used to reduce the background is called Isolation. In real $B$ events the $B$ daughter tracks carry a majority of the energy in the direction of the $B$ meson. Thus, there is on average little track activity in the vicinity of the $B$ hadron decay. In general, QCD $B$ candidates carry a smaller percentage of the momentum. This information can be used to remove background events. Isolation is defined as:

$$
\operatorname{Isol}(B)=\frac{P_{T}(B)}{P_{T}(B)+\sum_{i} P_{T}(i)}
$$

for all non- $B$ daughter tracks inside a cone $\Delta R<1$ around the $B$ candidate, where $\Delta R=$ $\sqrt{\left(\Delta \eta^{2}\right)+\left(\Delta \phi^{2}\right)}$. The definition of the cone $\Delta \mathrm{R}$ is illustrated in Figure 19. The Wonte Carlo distribution of the Isolation variable is shown in Figure 20. The isolation of the $B$ meson is required to be greater than 0.65 .

\subsection{Higher Order $B$ Decays}

The branching ratio for $B \rightarrow D^{*} \pi$ decay is approximately equal to the $B \rightarrow D \pi$ decay rate[1]. The $D^{*}$ meson decays via the $D \rightarrow D \pi$ or $D \rightarrow D \gamma$ channels. The reconstruction of $B$ candidates that decayed through the $B \rightarrow D^{*} \pi^{0}$ mode results in a invariant mass less then the nominal $B$ mass since the neutral pion or photon is not included in the invariant mass. The Monte Carlo distribution of the $B \rightarrow D \pi$ and $B \rightarrow D^{*} \pi$ decays reconstructed via 
the $B \rightarrow D \pi$ mode is shown in Figure 21 .

\subsection{Optimization Procedure}

The selection criteria are varied such that the ability to observe a small signal is maximized. To reduce bias, the optimization is performed without regard to the number of events in the signal region in the actual data. Instead, Monte Carlo events are used as our reference, $S$, sample and are scaled to the expected number of signal events in data. Since there is not a reliable Monte Carlo simulation which models our backgrounds, the sideband regions in data are used as the background, $B$, sample.

The figure-of-merit in the optimization procedure is the significance, defined as $S / \sqrt{B}$. The selection critera with the largest corresponding $S / \sqrt{B}$ value are the optimal cut values. To avoid a bias from selecting a low fluctuation in the background, the background is separated into even and odd alternating bins of width $10 \mathrm{MeV} / c^{2}$. In addition, the $B \rightarrow D^{*} \pi$ mass window is not included in the background region. The optimization is performed on the even bins and the final background fit is performed using the odd bins. The final selection cuts and mass plots are shown in the following sections.

\subsection{1 $B^{+} \rightarrow \overline{D^{0}} \pi^{+}, \overline{D^{0}} \rightarrow K^{+} \pi^{-}$}

Three final state tracks are combined to form a $B^{+}$candidate. The optimized selection requirement for $B^{+}$events are shown in Table 3 . The mass of the $B^{+}$candidates for election and muon events are shown in Figure 22. 


\begin{tabular}{c|ccc|c} 
Variable & Min & Max & Step Size & Optimized Value \\
\hline \hline$P_{T}(B)[\mathrm{GeV} / c]$ & 5.0 & 15.0 & 1 & 9.0 \\
$P_{T}(D)[\mathrm{GeV} / c]$ & 1.0 & 5.0 & .5 & 2.0 \\
$c \tau(B)[\mu \mathrm{m}]$ & 60 & 160 & 10 & 100 \\
$c \tau(D)[\mu \mathrm{m}]$ & -90 & 90 & 20 & -30
\end{tabular}

Table 3: The range of values and step size for each variable used in the optimization. The $B^{ \pm}$optimized selection criteria are found in the last column..

\subsection{2 $B^{0} \rightarrow D^{-} \pi^{+}, D^{-} \rightarrow K^{+} \pi^{-} \pi^{-}$}

Four final state tracks are combined to form a $B^{0}$ candidate. The selection requirements for $B^{0}$ events are shown in Table 4. The mass of the $B^{0}$ candidates for electron and muon events are shown in Figure 22. 


\begin{tabular}{c|ccc|c} 
Variable & Min & Max & Step Size & Optimized Value \\
\hline$P_{T}(B)[\mathrm{GeV} / c]$ & 5.0 & 15.0 & 1 & 8.0 \\
$P_{T}(D)[\mathrm{GeV} / c]$ & 1.0 & 5.0 & .5 & 2.0 \\
$c \tau(B)[\mu \mathrm{m}]$ & 60 & 160 & 10 & 140 \\
$c \tau(D)[\mu \mathrm{m}]$ & -90 & 90 & 20 & 10
\end{tabular}

Table 4: The range of values and step size for each variable used in the optimization. The $B^{0}$ optimized selection criteria are found in the last column. 


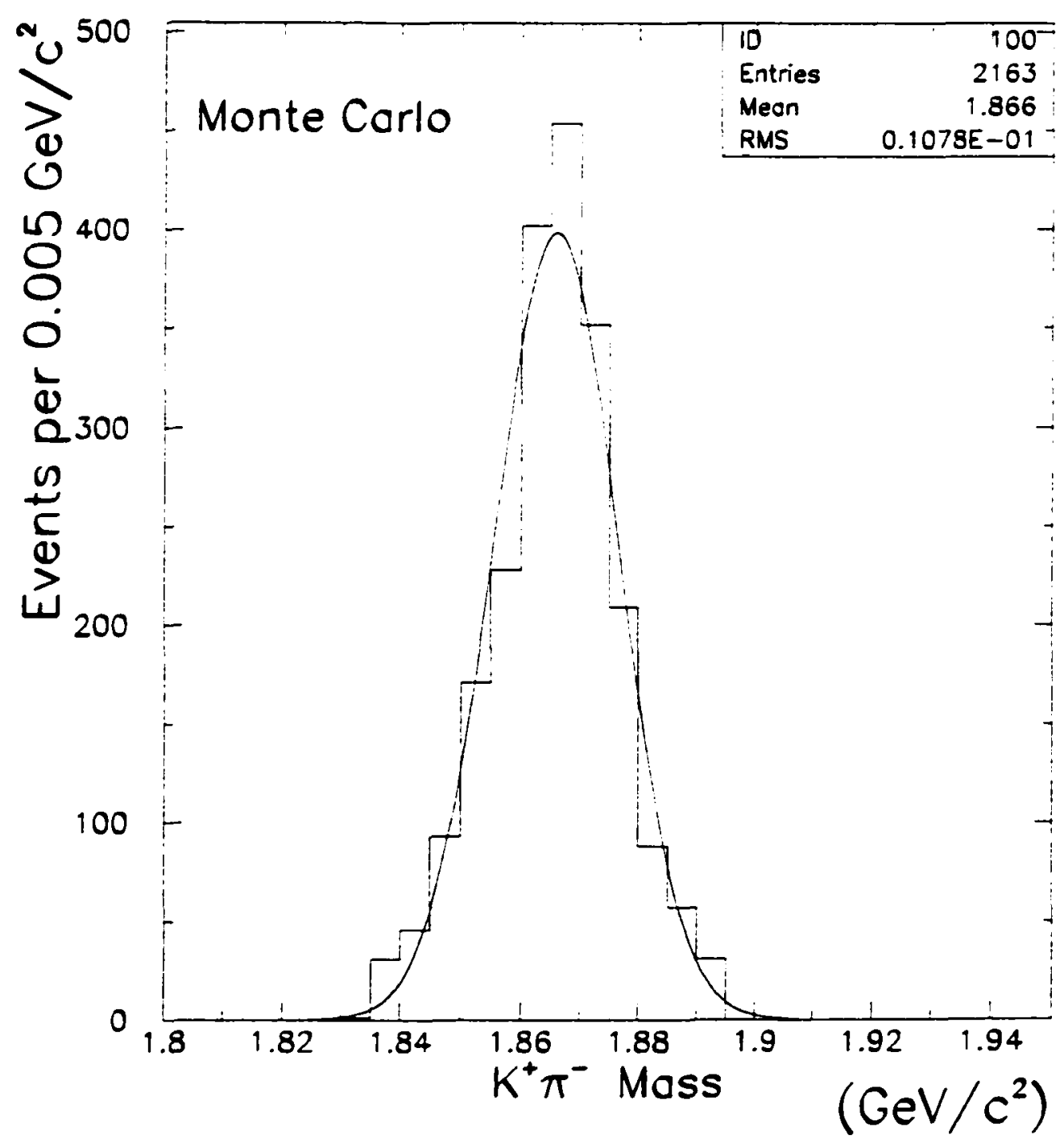

Figure 14: The reconstructed mass for $\bar{D}^{0} \rightarrow K^{+} \pi^{-}$Monte Carlo events. 


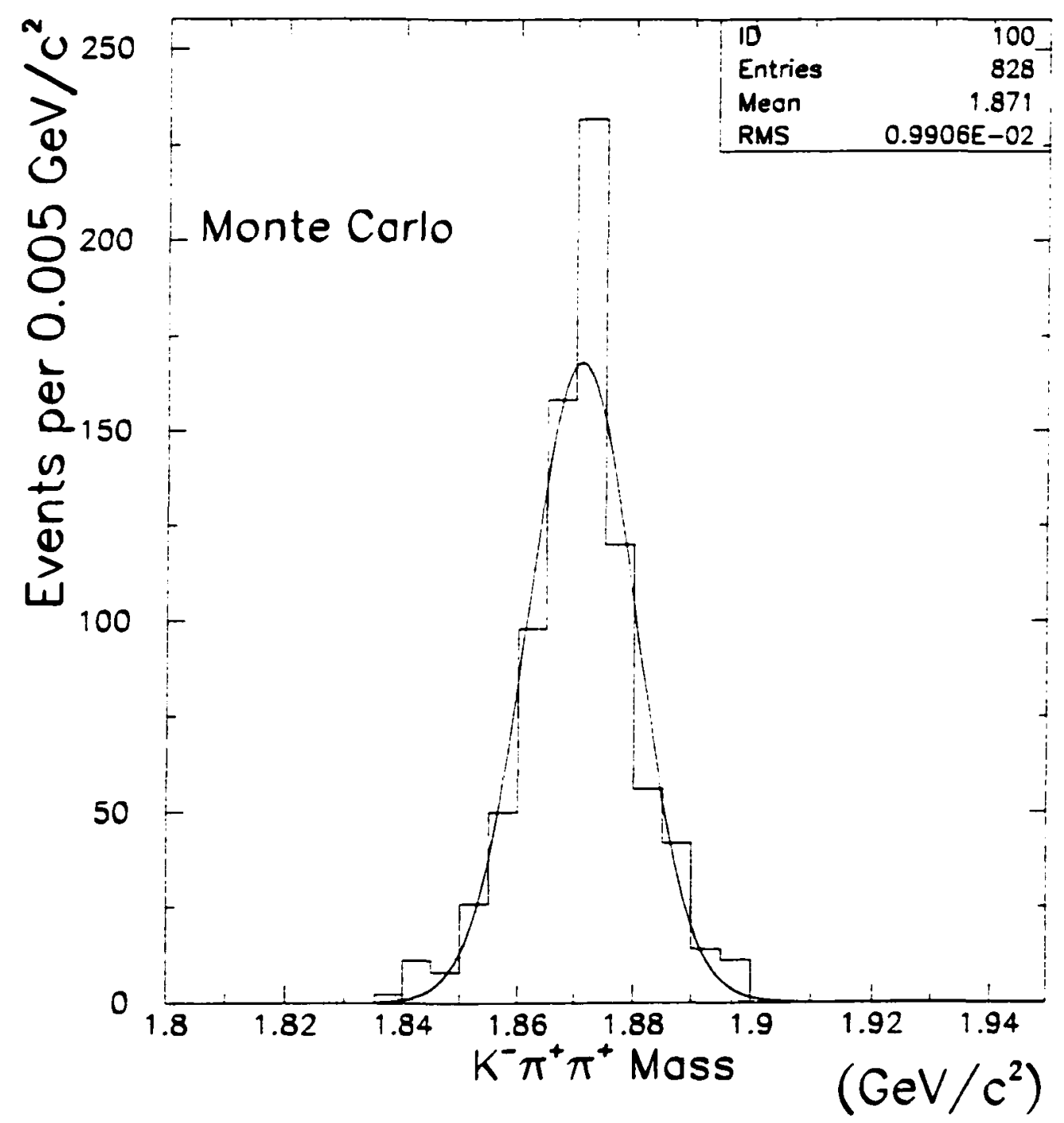

Figure 15: The reconstructed mass for $D^{-} \rightarrow K^{+} \pi^{-} \pi^{-}$Monte Carlo events. 


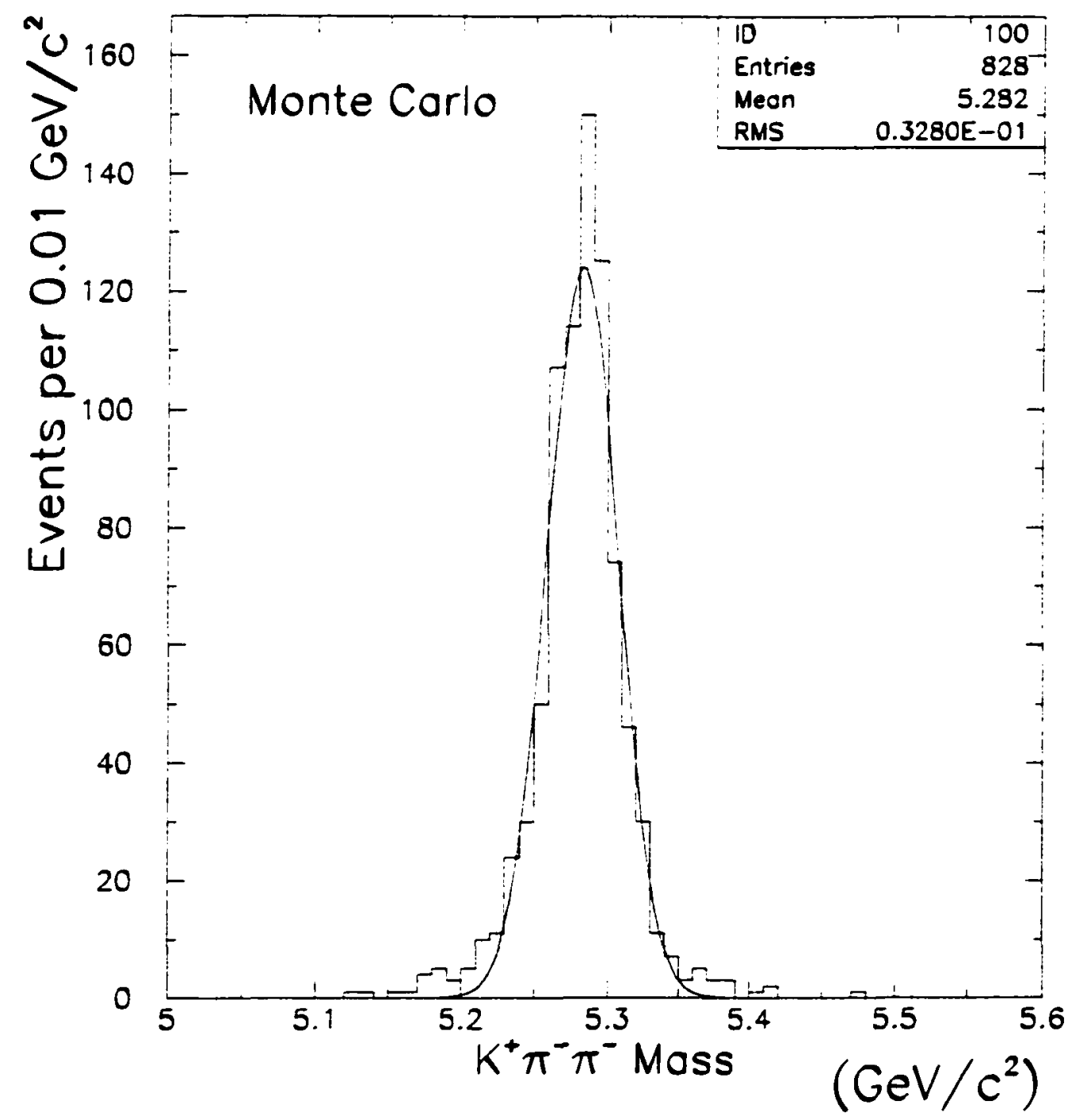

Figure 16: The reconstructed mass for $B^{0} \rightarrow D^{-} \pi^{+}$Monte Carlo events. 


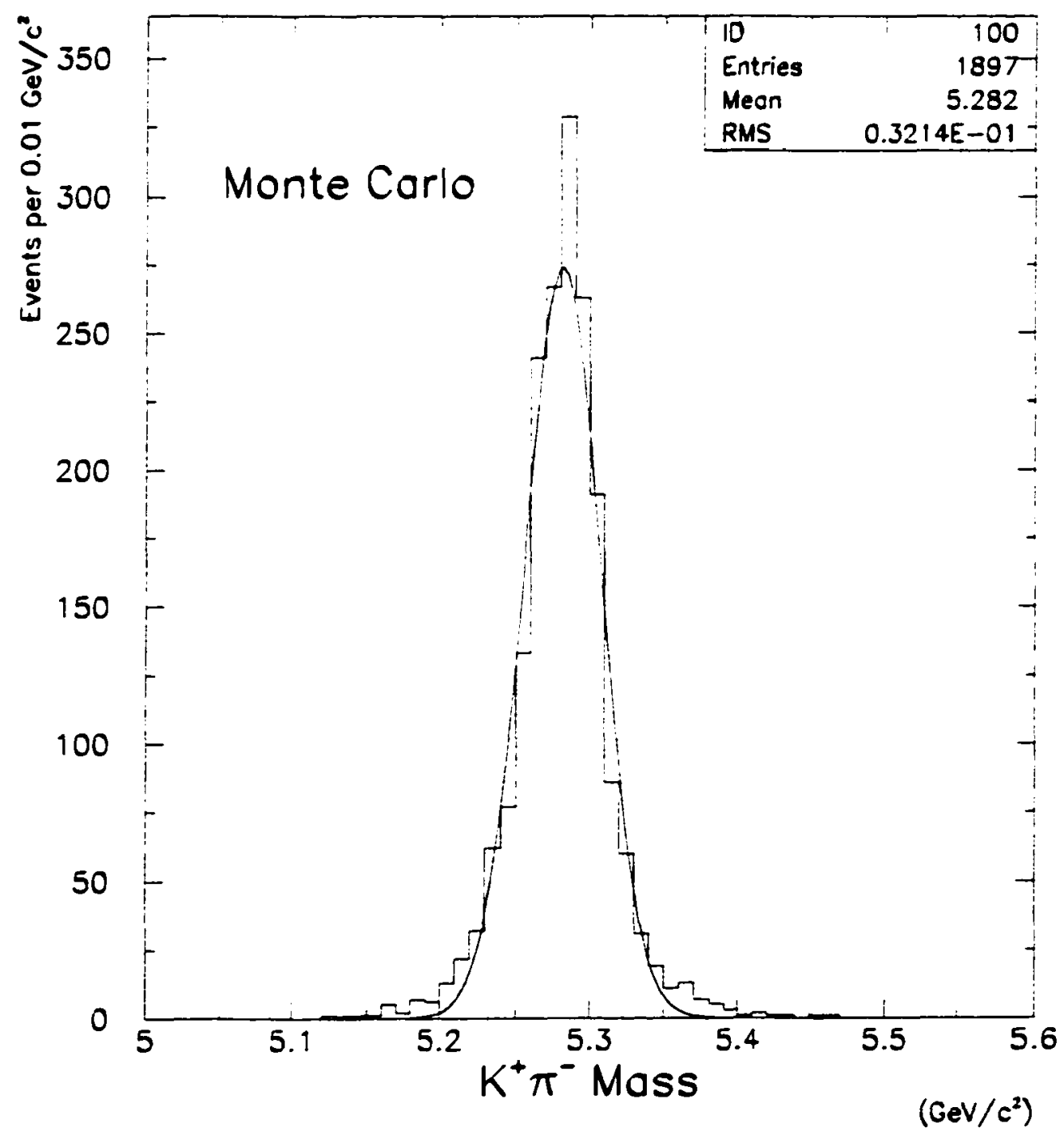

Figure 17: The reconstructed mass for $B^{+} \rightarrow \bar{D}^{0} \pi^{+}$Monte Carlo events. 

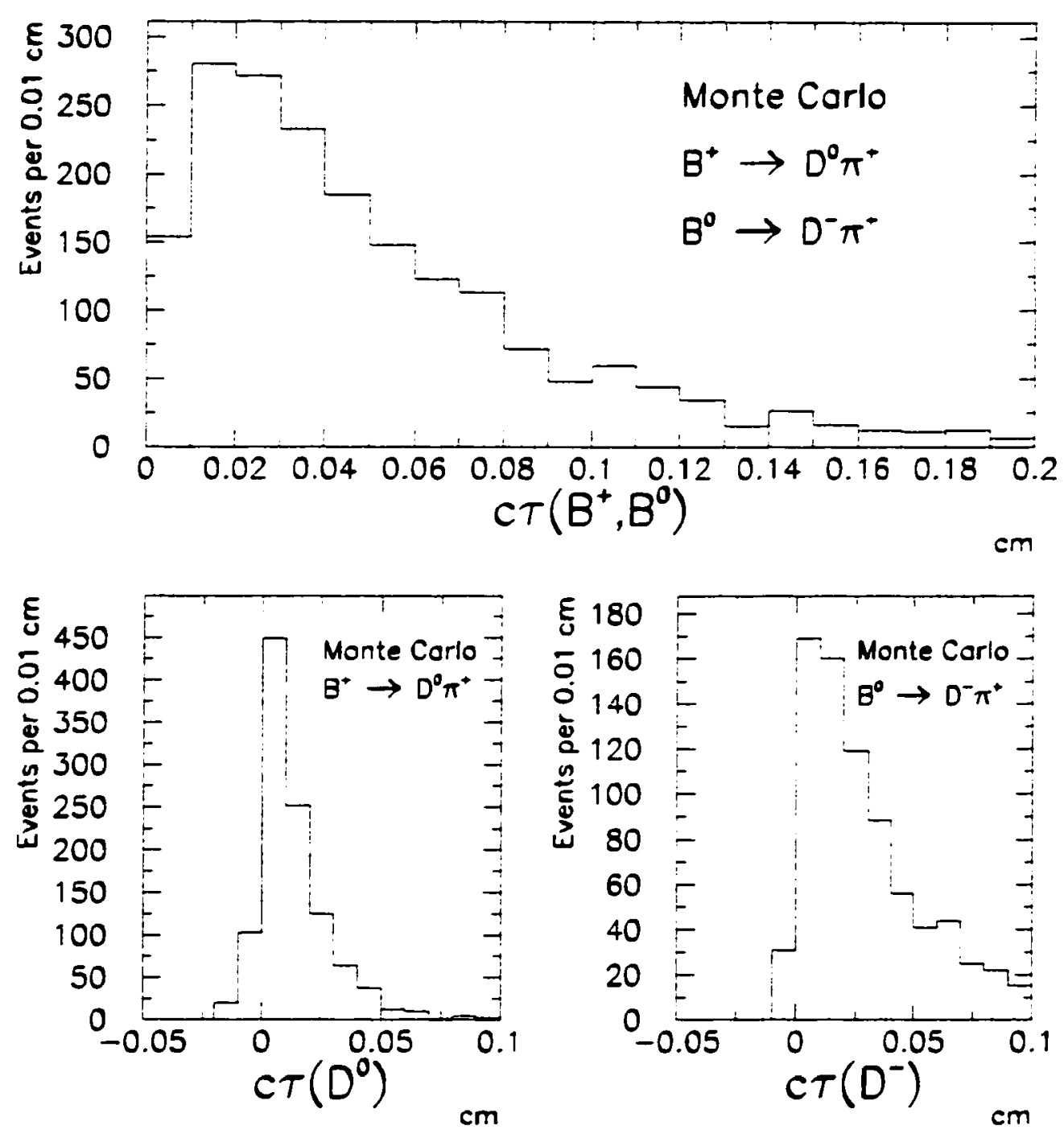

Figure 18: Monte Carlo distribution of $B$ and $D$ mesons proper decay time in $B^{+} \rightarrow$ $\overline{D^{0}} \pi^{+}, \overline{D^{0}} \rightarrow K^{+} \pi^{-}$and $B^{0} \rightarrow{D^{-}}^{+}, D^{-} \rightarrow K^{+} \pi^{-} \pi^{-}$decay modes. The top plot is the Monte Carlo proper decay time distribution of $B$ mesons. The bottom left plot is the Monte Carlo proper decay time distribution of the $\bar{D}^{0}$ meson. The bottom right plot is the Monte Carlo proper decay time distribution of the $D^{-}$meson 


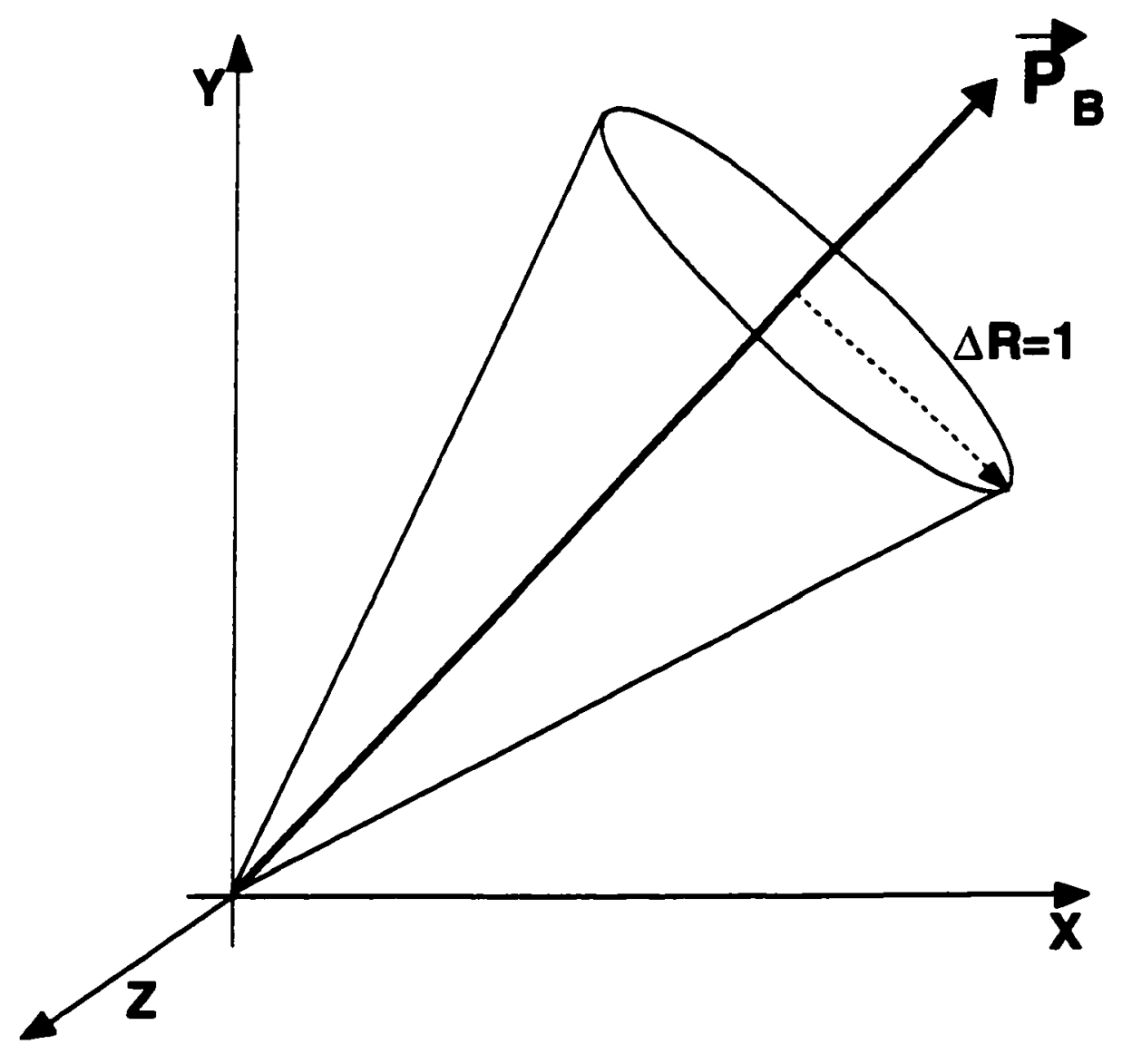

Figure 19: Definition of the cone around the $B$ meson used in the isolation calculation. 


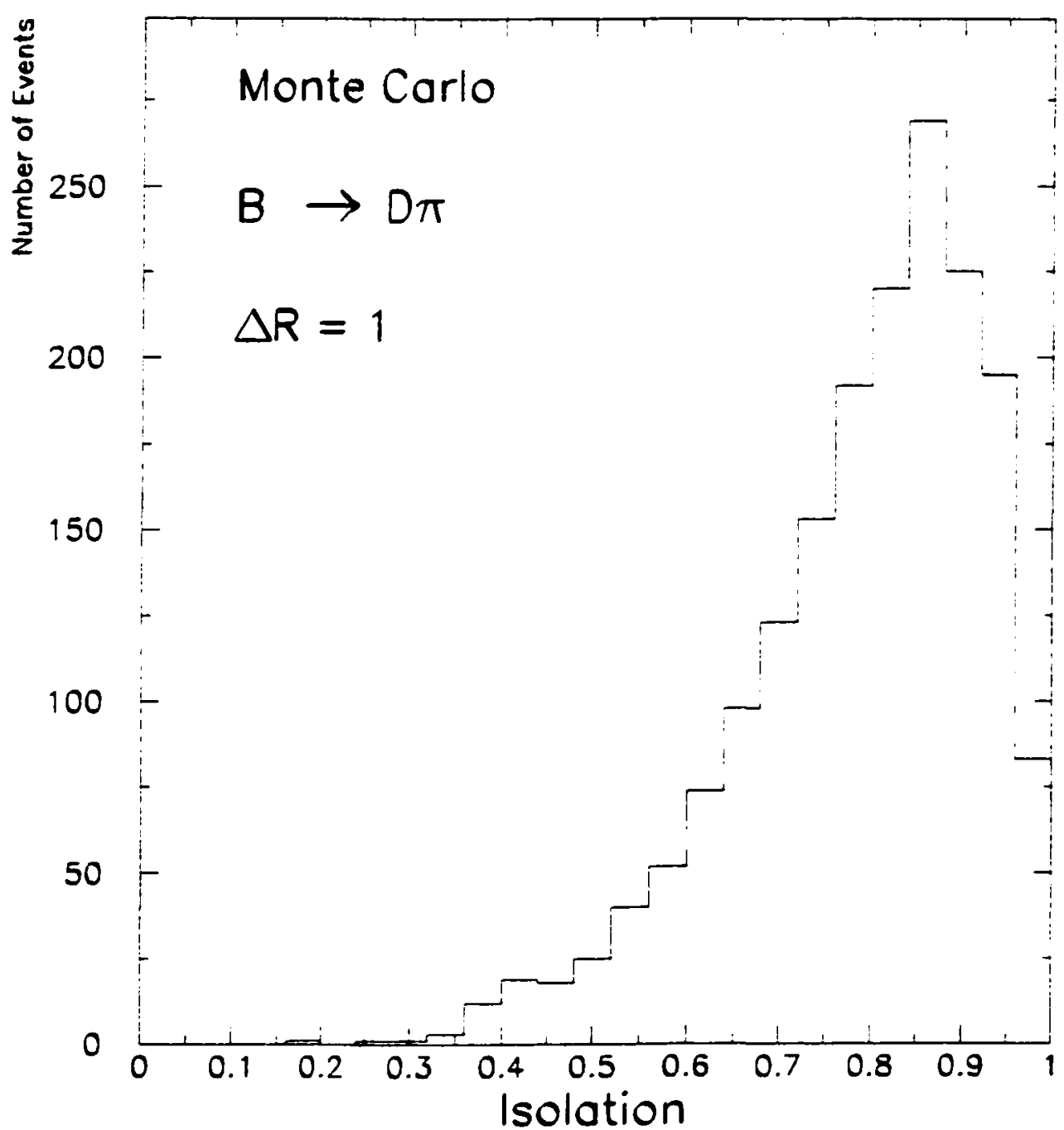

Figure 20: Monte Carlo distribution of the isolation variable in $B^{+} \rightarrow \overline{D^{0}} \pi^{+}, \overline{D^{0}} \rightarrow K^{+} \pi^{-}$and $B^{0} \rightarrow D^{-} \pi^{+}, D^{-} \rightarrow K^{+} \pi^{-} \pi^{-}$decay. 


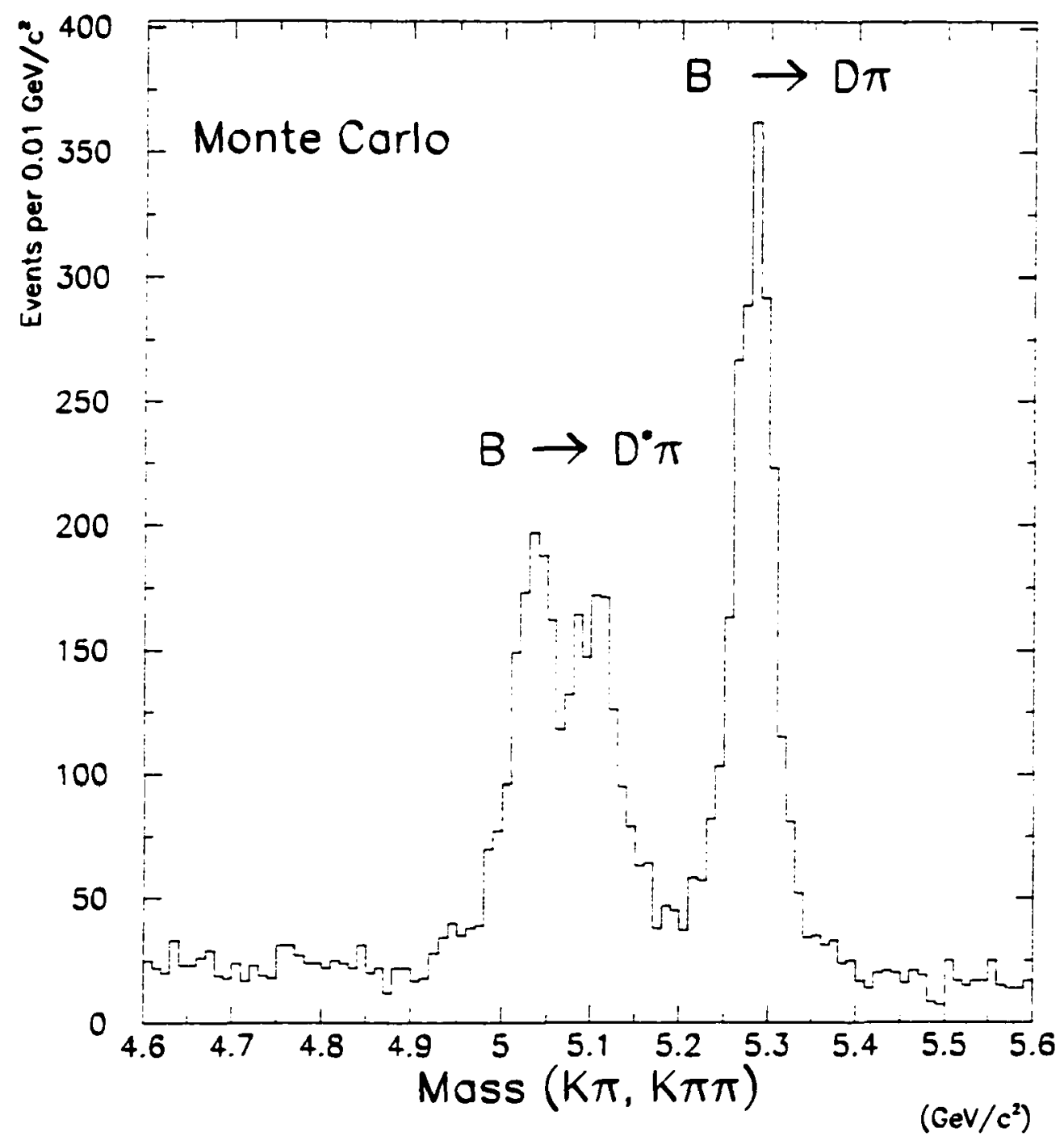

Figure 21: The Monte Carlo mass distribution of $B \rightarrow D^{(\cdot)} \pi$ candidates reconstructed in $B^{+} \rightarrow \overline{D^{0}} \pi^{+}, \overline{D^{0}} \rightarrow K^{+} \pi^{-}$and $B^{0} \rightarrow D^{-} \pi^{+}, D^{-} \rightarrow K^{+} \pi^{-} \pi^{-}$modes. The left peak represents the $B \rightarrow D^{*} \pi$ decays (reconstructed as $B \rightarrow D \pi$ ) and the right peak represents the $B \rightarrow D \pi$ decays. 


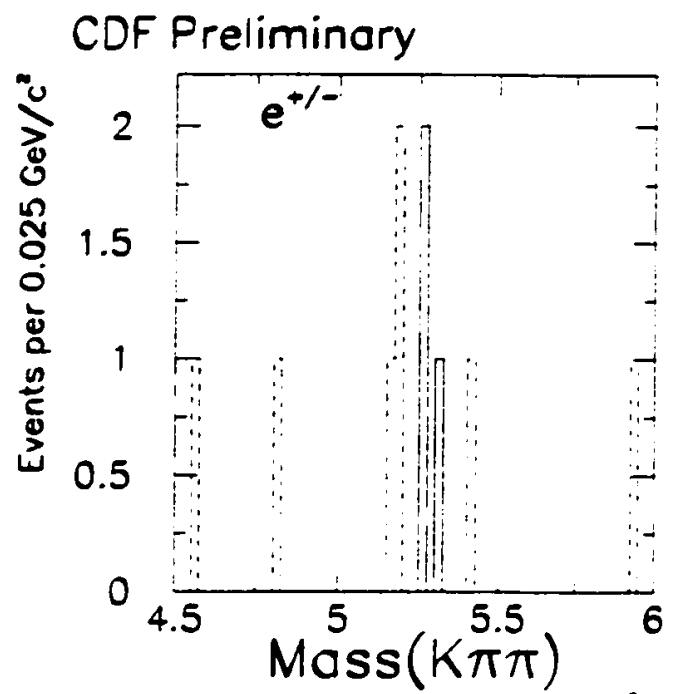

$\left(\mathrm{GeV} / \mathrm{c}^{2}\right)$
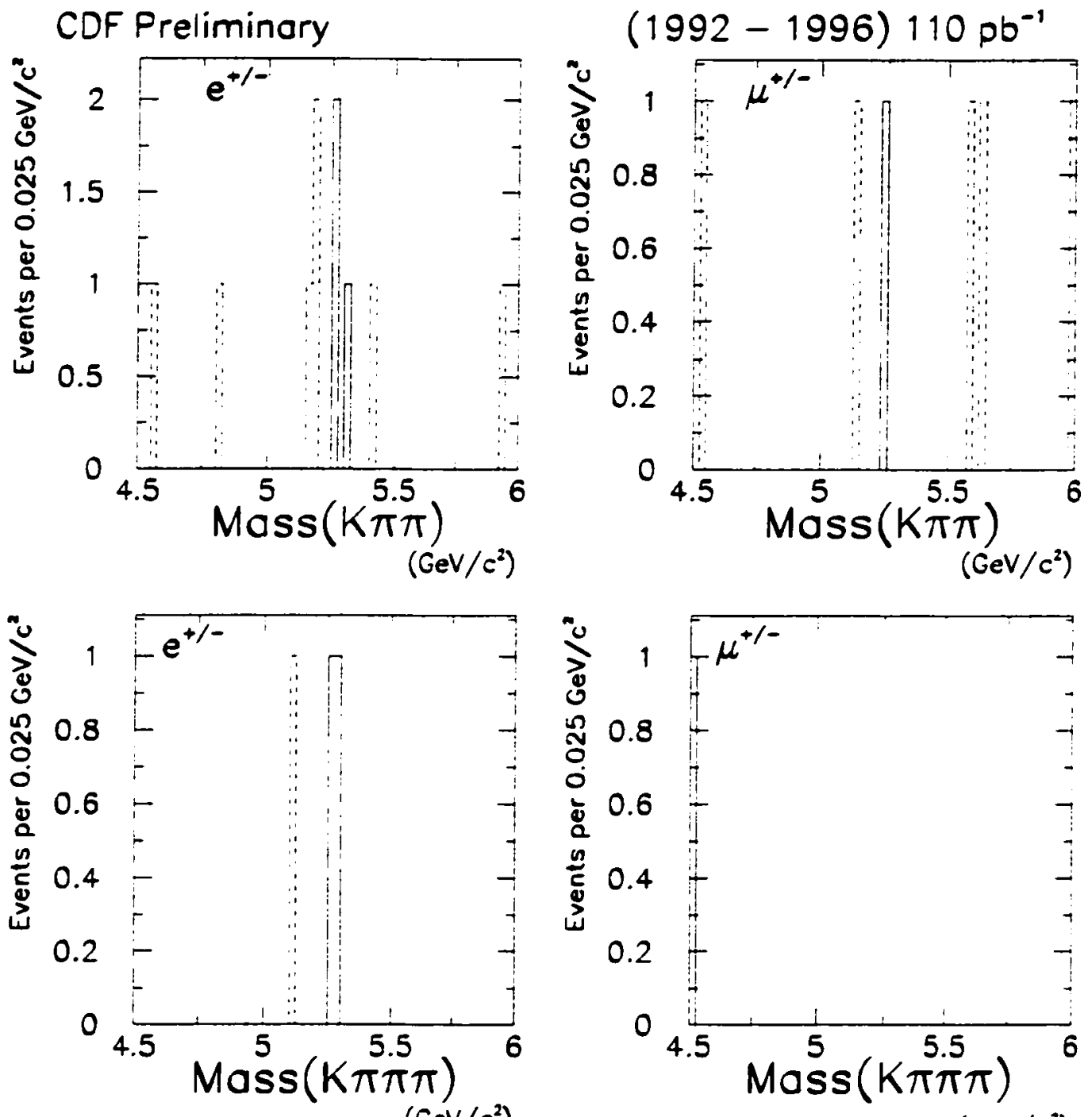

$\left(\mathrm{GeV} / \mathrm{c}^{2}\right)$

Figure 22: These four plots show the mass of the reconstructed $B$ meson candidates. The top row shows mass of $B^{+} \rightarrow \overline{D^{0}} \pi^{+}, \overline{D^{0}} \rightarrow K^{+} \pi^{-}$candidates for electron (left) and muon (right). The bottom row shows the mass of $B^{0} \rightarrow D^{-} \pi^{+}, D^{-} \rightarrow K^{+} \pi^{-} \pi^{-}$candidates. The solid histogram represents $B$ candidates in the signal region and the dashed histogram represents $B$ candidates outside the signal region. 


\subsection{3 $B^{+}$and $B^{0}$ Combined Result}

Due to the limited number of statistics in each $B$ decay mode, the electron and muon events for both $B^{+}$and $B^{0}$ candidates are combined. The mass of the combined $B^{+} \rightarrow$ $\overline{D^{0}} \pi^{+}, \overline{D^{0}} \rightarrow K^{+} \pi^{-}$and $B^{0} \rightarrow D^{-} \pi^{+}: D^{-} \rightarrow K^{+} \pi^{-} \pi^{-}$candidates is shown in Figure 23 . The fitting method and estimates of the number of signal and background events are described in the following sections.

\subsubsection{Fitting Method}

Based on the mass distribution of the combined sample, the number of signal events can be determined by fitting for the number of signal and background events. A binned fit is reliable, but less powerful, method in principle. A more powerful method is using a unbinned fit method. An unbinned extended maximum log-likelihood fit is used to determine the number of signal events. The mass distribution is fit to a Gaussian distribution, $f_{s i g}$, for the signal events, plus a linear distribution, $f_{b a c k}$, to describe the background. A Poisson term in the total number of events is included to correctly account for uncertainties in the fit. The fit is extended over the mass region 4.5 to $6.0 \mathrm{GeV} / \mathrm{c}^{2}$. Specifically, the likelihood function is a product of the probability distribution of the invariant mass of each event in the region:

$$
L=e^{-\left(n_{s}+n_{b}\right)} \times \frac{\left(n_{s}+n_{b}\right)^{N}}{N !} \Pi_{i=1}^{N}\left(\frac{n_{s} \cdot f_{s i g}+n_{b} \cdot f_{b a c k}}{n_{s}+n_{b}}\right)
$$

where $N$ is the total number of events in the mass region $4.5<m_{i}<6.0 \mathrm{GeV} / c^{2}$ and $n_{s}$ and $n_{b}$ are the measured number of signal and background events. The signal region is described 
by a normalized Gaussian function, $f_{s i g}$, that has the form:

$$
f_{s i g}=\frac{1}{\sqrt{2 \pi} \sigma_{i}} e^{-\frac{1}{2}\left(\frac{\left.m_{1}-M_{B}\right)^{2}}{\sigma_{i}},\right.}
$$

are $M_{B}$ is the nominal $B$ mass and $m_{i}$ is the event mass and $\sigma_{i}$ is the uncertainty in the mass fit. The background function, $f_{\text {back }}$, is a first-order polynomial:

$$
f_{\text {back }}\left(m_{i}\right)=a m_{i}+b
$$

where $a$ is the slope and $b$ is the $y$-intercept. The background function must be normalized:

$$
\int_{m_{i}}^{m_{u}} f_{b a c k}\left(m_{i}\right) d m_{i}=1
$$

where $m_{u}$ is upper limit of the mass range and $m_{l}$ is the lower limit of the mass range. The likelihood function, $L$, is maximized to determine the free parameters. When the fit is performed with the mean of the Gaussian fixed to the nominal $B$ mass, the maximimum likelihood fit yields $4.2_{-2.1}^{+2.9}$ signal events with an expected background is $0.85_{-0.29}^{+0.29}$ events. If the fit is peformed with the mean allowed to float, the maximimum likelihood fit yields $4.1_{-2.1}^{+2.7}$ events and a mean of $5.274_{-0.013}^{+0.015} \mathrm{GeV} / c^{2}$ with an expected background of $0.89_{-0.3}^{+0.3}$ events. Since the best fit mean is within one sigma of the nominal $B$ mass the deviation is most probably a statistical fluctuation. The results quoted are based on the fit with the constrained mean. Both methods give similar final results, which are equivalent given the error on the number of signal events. The signal represents a $4.1 \sigma$ deviation from the expected background. 


\subsubsection{Signal Cross Checks}

There are many methods to determine the validity of the signal. Feldman and Cousins propose a single algorithm that decides if a one-side or double-sided limit should be quoted [38]. The Feldman and Cousins $95 \%$ confidence interval for 4.2 signal events over an expected background of 0.85 events is $[0.5,8.9]$. Thus, the null hypothesis is eliminated at the $95 \%$ confidence level.

Since the events in the signal region are not used in the optimization process, there should be no inherent bias. However, several checks are performed to determine if the signal peak is real. The selection criteria are varied around their optimized values to measure the signals dependence on the specific cut values. The number of signal events varies around the optimized values, but the excess remains.

The normalized mass, $\frac{\left(m_{i}-M_{B}\right)}{\sigma_{m_{i}}}$, for the electron and muon events in each $B$ decay mode is shown in Figure 24 and 25 . The $\sigma_{m_{i}}$ term is equal to the uncertainty returned from the likelihood fit scaled by a factor of 1.3. An unbinned likelihood fit of the normalized mass is performed with a width of 1 and a mean of 0.0 . The number of events returned from the normalized mass plot gaussian fit is $4.9_{-2.0}^{+2.3}$. This is consistent with the result of the unbinned likelihood fit of the $B$ candidate mass.

Another check of the validity of the signal is to examine the distribution of the $B$ and $D$ meson properties. The proper decay time of $B^{+} \rightarrow \overline{D^{0}} \pi^{+}, \overline{D^{0}} \rightarrow K^{+} \pi^{-}$and $B^{0} \rightarrow$ $D^{-} \pi^{+}, D^{-} \rightarrow K^{+} \pi^{-} \pi^{-}$candidates are shown in Figures 26 and 27 . The agreement of the shape of these distributions with Monte Carlo is reasonable. 


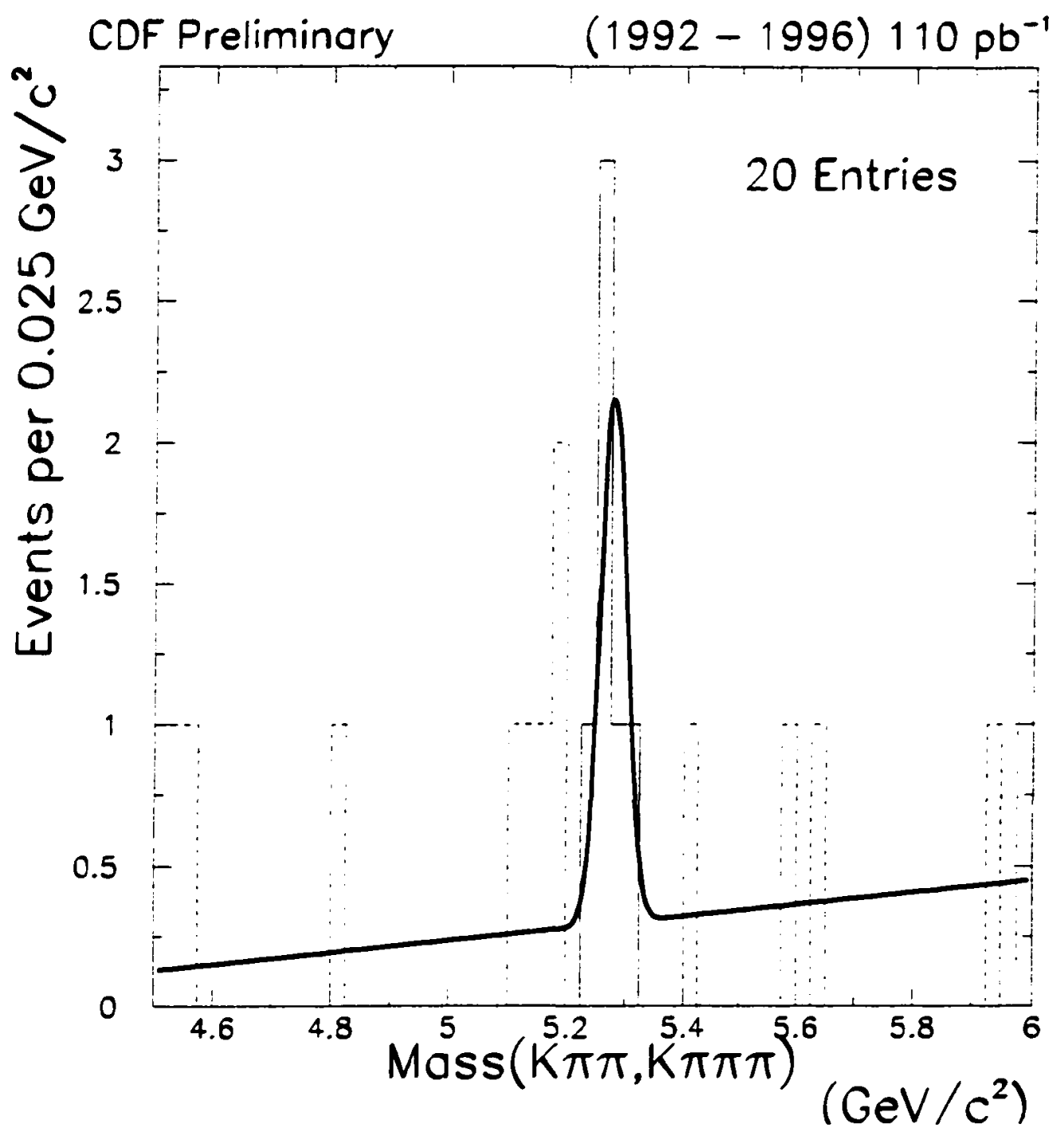

Figure 23: The combined mass of the $B^{0}$ and $B^{+}$candidates for electron and muon data. The Gaussian and linear function plotted on the data use the parameters returned from the the extended log-likelihood fit. The width of the Gaussian is equal to the average uncertainty in the mass fit scaled by a factor of 1.3 . The solid histogram represents $B$ candidates in the signal region and the dashed histogram represents $B$ candidates outside the signal region. 

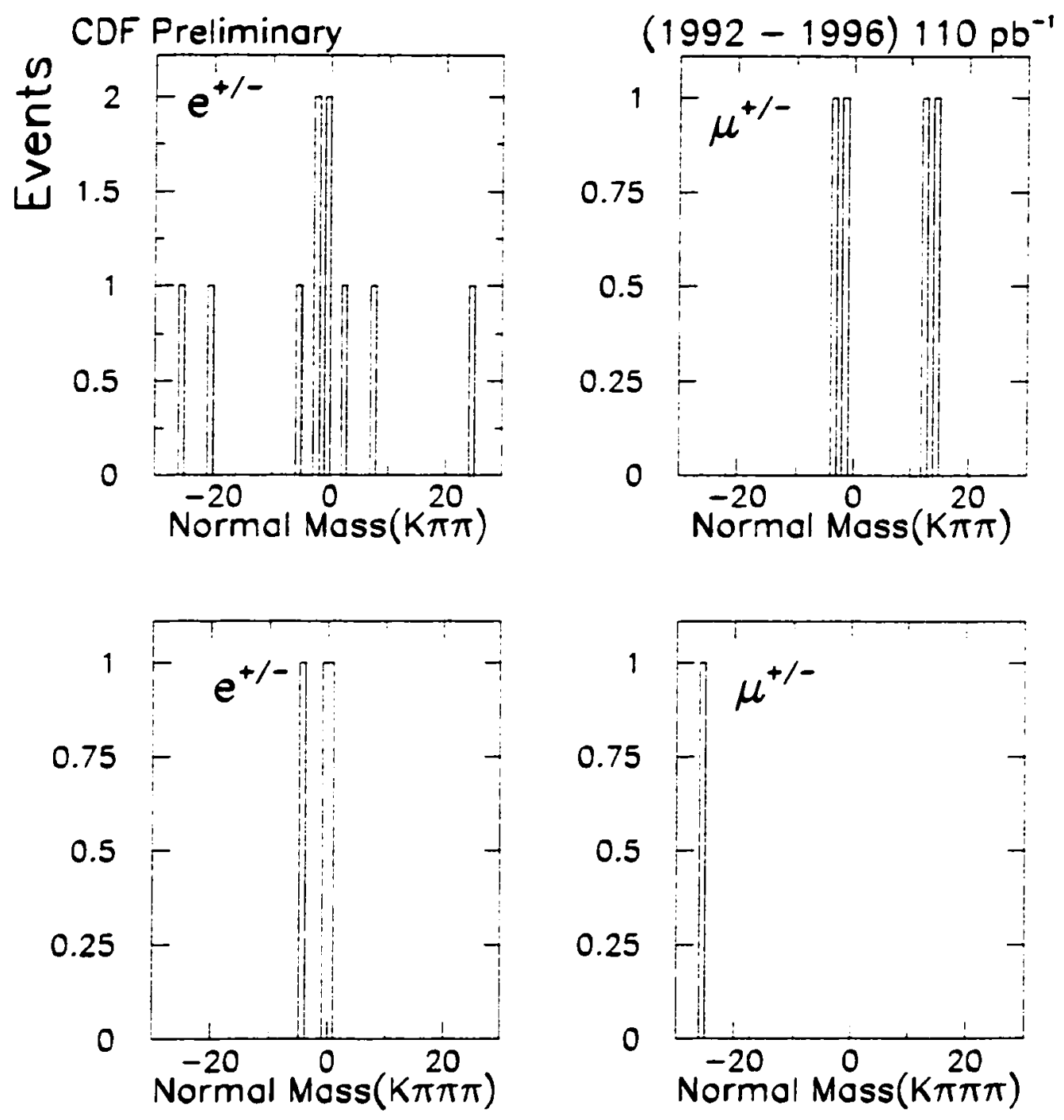

Figure 24: These four plots show the normalized mass of the $B$ candidates. The top row shows the normalized mass of the $B^{+} \rightarrow \overline{D^{0}} \pi^{+}, \overline{D^{0}} \rightarrow K^{+} \pi^{-}$events for electron (left) and muon (right) events. The bottom row shows the normalized mass of the $B^{0} \rightarrow D^{-} \pi^{+}, D^{-} \rightarrow$ $K^{+} \pi^{-} \pi^{-}$events. 


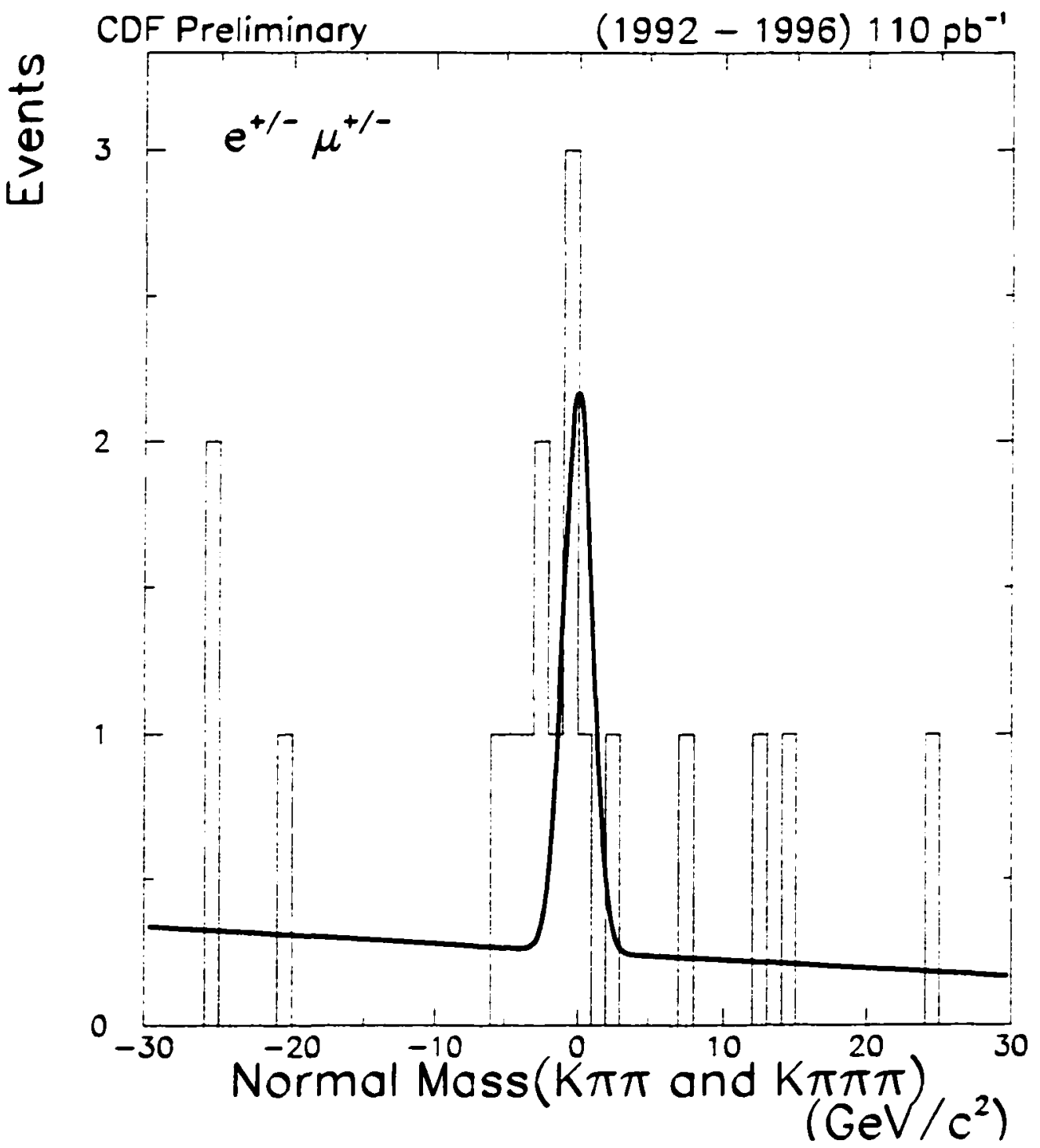

Figure 25: The combined normalized mass of the electron and muon $B^{+} \rightarrow \overline{D^{0}} \pi^{+}, \overline{D^{0}} \rightarrow$ $K^{+} \pi^{-}$and $B^{0} \rightarrow D^{-} \pi^{+}, D^{-} \rightarrow K^{+} \pi^{-} \pi^{-}$candidates. 


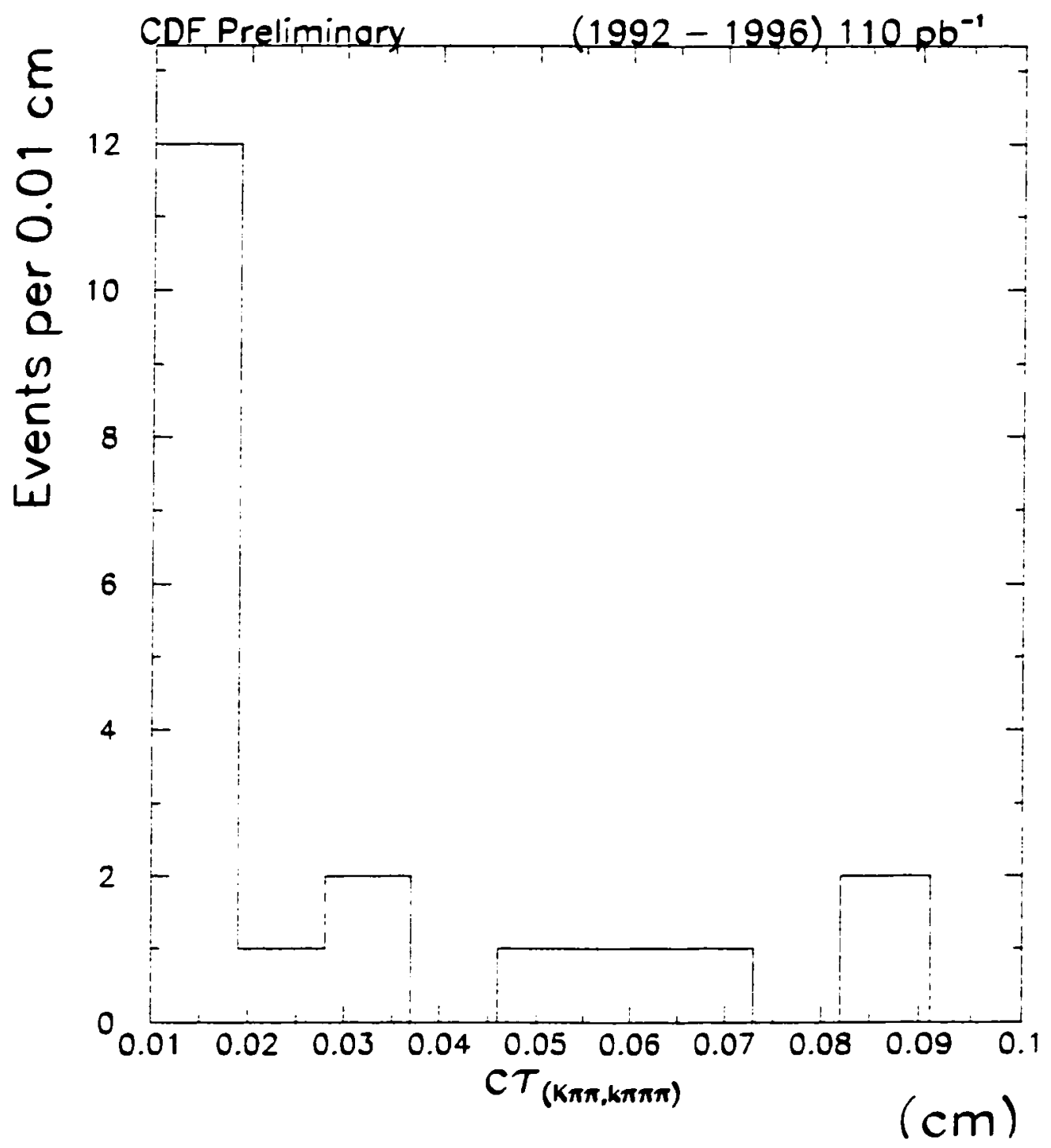

Figure 26: The proper decay time of the combined $B^{+}$and $B^{0}$ candidates. 


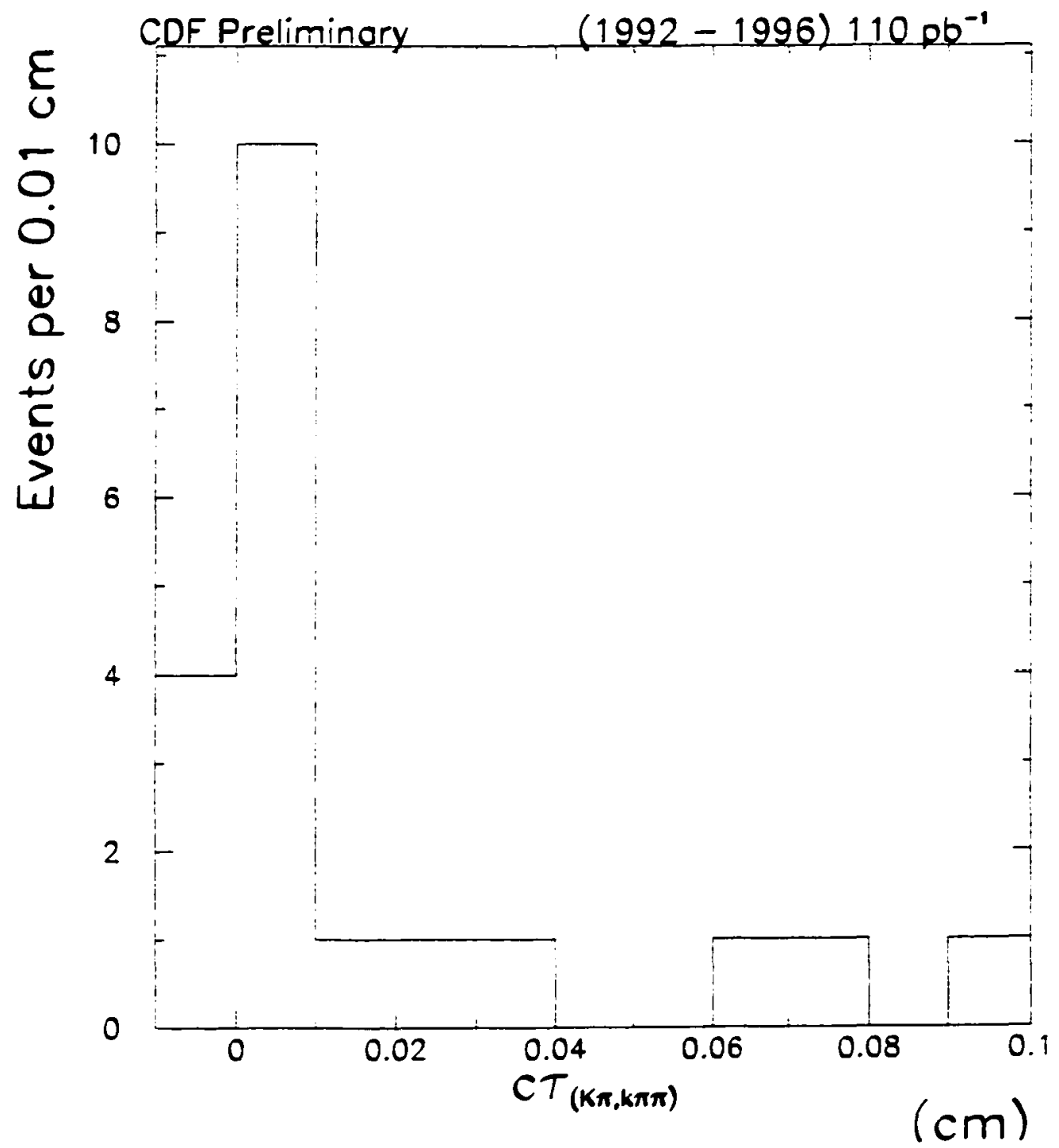

Figure 27: The proper decay length of the combined $D^{+}$and $\bar{D}^{0}$ candidates. 


\subsection{Measurement of $b \bar{b}$ Fraction}

$b \bar{b}$ and $c \bar{c}$-quarks are produced in pairs in $p \bar{p}$ collisions. The lepton sample is a result of many semileptonic decay processes originating from $b \bar{b}, c \bar{c}$ and other processes. By measuring the number of $B^{+}$and $B^{0}$ mesons in the lepton sample, the fraction of $b \bar{b}$ events in the total lepton sample can be determined. The $b$ and $c$-quarks fragment independently into different $B$ and $D$ hadron flavors and the subsequent decay of the hadrons is also independent. All $B$ and $D$ hadrons can decay semileptonically:

$$
b \rightarrow \bar{B} X \rightarrow \ell^{-} X
$$

or

$$
c \rightarrow \bar{D} X \rightarrow \ell^{-} X
$$

If the $b$ and $c$-quarks fragment into a neutral $B$ or $D$ hadron, the neutral hadron could mix before decaying semileptonically:

$$
b \rightarrow \bar{B}^{0} \rightarrow B^{0} \rightarrow \ell^{+} X
$$

thus there is not lepton charge correlation requirement between the charge of the reconstructed meson and charge of the lepton. In addition, a fraction of the events in the lepton sample also originate from other hadrons most notably sequential decays:

$$
b \rightarrow \bar{B} \rightarrow X \rightarrow \ell^{+} X
$$

Thus, the $b \bar{b}$ fraction measure will include leptons from $B$ semileptonic decay (mixed or unmixed) and sequential decays. 
Since $b$-quarks are produced in pairs, if there is a lepton on one hemisphere then the other $B$ hadron's decay products are most likely in the opposite hemisphere ${ }^{9}$. The efficiency calculates the fraction of $B$ reconstructed in the detector after the selection criteria have been applied. Based on the measurement of the number of $B^{+}$and $B^{0}$ signal events, $N_{\text {signal }}$, the $b \bar{b}$ fraction can be calculated from the following equation:

$$
b \bar{b}_{\text {fraction }}=\frac{N_{\text {signal }}}{N_{\text {total }}} \times \frac{1}{\left(f_{d} \times B R\left(B^{0}\right) \times \epsilon\left(B^{0}\right)\right)+\left(f_{u} \times B R\left(B^{+}\right) \times \epsilon\left(B^{+}\right)\right)},
$$

where $N_{\text {total }}$ is the number of lepton events after basic lepton quality cuts, $f_{u}$ and $f_{d}$ are the $b$-quark fragmentation fractions, and $\operatorname{BR}(B)$ are the $B$ meson product branching ratio, and $\epsilon(B)$ is the reconstruction efficiency for a $B$ meson in the opposite hemisphere of the letpon. The factors used in this calculation can be found in Table 5. Based on the $4.2 B^{+}$and $B^{0}$ signal events reconstructed, the $b \bar{b}$ fraction is determined to be $27 \pm 14.8 \pm 5.6 \%$.

\section{The Search for $B_{s} \rightarrow D_{s} \pi$}

Based on spectator decay diagrams, $B_{s}^{0} \rightarrow D_{s}^{-} \pi^{+}$decays are expected to occur at the same rate as $B^{0} \rightarrow D^{-} \pi^{+}$. However, due to the lower probability of the $b$-quarks to fragment into $B_{s}$ mesons compared to $B_{d}$ mesons, the number of $B_{s}$ mesons in our data sample is expected to be smaller then the number of $B_{d}$ mesons. The numerous decay modes of the $D_{s}$, with relatively equal branching ratios, results in a more complicated $B_{s}$ reconstruction. The six modes are chosen based on the relative branching ratio and the number of final state tracks. The six $D_{s}$ decay modes and the basic reconstruction cuts are introduced in Section 11.1.

\footnotetext{
${ }^{9}$ Though not necessarily in the detectors acceptance
} 


\begin{tabular}{|c|c|}
\hline$N_{\text {total }}$ & 6429302 \\
\hline$N_{\text {signal }}$ & $4.2 \pm 2.1$ \\
\hline$f_{u}$ & $38.9 \pm 4.8 \%$ \\
\hline$f_{d}$ & $38.9 \pm 5.4 \%$ \\
\hline $\mathrm{BR}\left(B^{+} \rightarrow \overline{D^{0}} \pi^{+}, \overline{D^{0}} \rightarrow K^{+} \pi^{-}\right)$ & $0.020 \pm 0.002 \%$ \\
\hline $\mathrm{BR}\left(B^{0} \rightarrow D^{-} \pi^{+}, D^{-} \rightarrow K^{+} \pi^{-} \pi^{-}\right)$ & $0.027 \pm 0.004 \%$ \\
\hline$e f f\left(B^{+}\right)$ & $2.0 \pm 0.1 \%$ \\
\hline$e f f\left(B^{0}\right)$ & $0.8 \pm 0.08 \%$ \\
\hline \hline$b \vec{b}_{\text {fraction }}$ & $(27.3 \pm 14.8 \pm 5.6) \times 10^{-2}$ \\
\hline
\end{tabular}

Table 5: The $b \bar{b}$ fraction in the inclusive lepton dataset.

The optimization procedure is described in Section 11.2. The resulting mass plots after the selection criteria have been applied and are shown in Section in 11.3. The combined mass plot is presented in Section 11.4. The combined mass plot using the average selection criteria is presented in Section 11.5.

\section{1 $B_{s}$ Reconstruction}

To enhance the probability of reconstructing $B_{s}$ mesons, multiple $D_{s}$ decay modes are studied. However, certain decay modes may have greater sensitivity then other modes. Thus, each mode is studied individually. After the optimization, the expected background and efficiency for different combinations of modes are studied to determine which combination achieves the best sensitivity. The $D_{s}$ modes studied in $B_{s}^{0} \rightarrow D_{s}^{-} \pi^{+}$are: 
$1 D_{s}^{-} \rightarrow \phi \pi^{-}, \phi \rightarrow K^{+} K^{-}$,

$2 D_{s}^{-} \rightarrow \phi \pi^{-} \pi^{-} \pi^{+}, \phi \rightarrow K^{+} K^{-}$,

$3 D_{s}^{-} \rightarrow K^{*^{0}} K^{-}, K^{*^{0}} \rightarrow K^{+} \pi^{-}$,

$4 D_{s}^{-} \rightarrow K_{s} K^{-}, K_{s} \rightarrow \pi^{+} \pi^{-}$

$5 D_{s}^{-} \rightarrow \overline{f^{0}} \pi^{-}, \overline{f^{0}} \rightarrow \pi^{+} \pi^{-}$,

$6 D_{s}^{-} \rightarrow K^{0^{0}} \pi^{-}, K^{*^{0}} \rightarrow K^{+} \pi^{-}$.

A summary of all the basic reconstruction cuts performed before the optimization cuts are:

- Probability of the chi-square for the vertex fit $>0.1 \%$ for $\phi, K_{s}, K^{0^{*}}, f^{0}, D$ and $B$ mesons,

- $P_{T}\left(\pi_{B}\right), P_{T}\left(K_{D}\right)>0.6 \mathrm{GeV} / c$,

- $P_{T}>0.4 \mathrm{GeV} / c$ for all other final state particles,

- $|\Delta z|<5 \mathrm{~cm}$ for all final state particles,

- Each final state track has greater then 3 SVX hits,

- Impact parameter significance with respect to the primary vertex, abs $\left|\frac{d_{0}}{\sigma_{d_{0}}}\right|,>3$,

- $1.9486<M\left(D_{s}\right)<1.9886 \mathrm{GeV} / c^{2}$. 


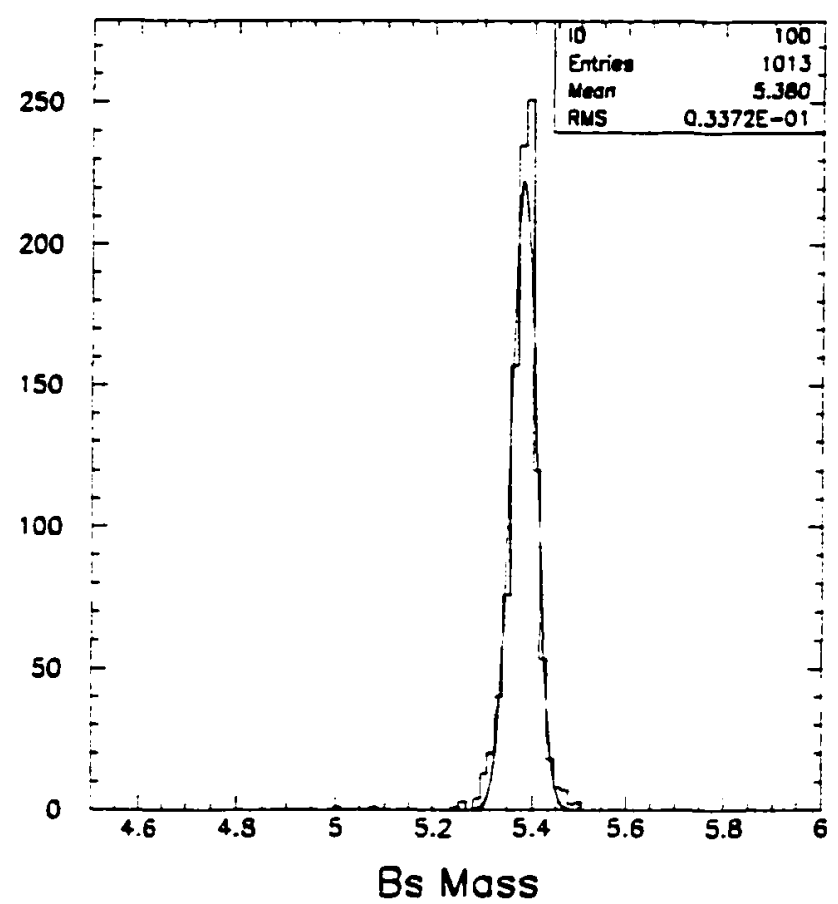

Figure 28: The reconstructed Monte Carlo mass of $B_{s}$ in all mode.

\subsection{Optimization Procedure}

Based on the number of $B^{+}$and $B^{0}$ reconstructed, and the respective fragmentation fractions and branching ratios, the probability of observing a significant $B_{s}^{0} \rightarrow D_{s}^{-} \pi^{+}$signal is unlikely. The optimization strategy is thus to select criteria which are most likely to place the lowest possible upper limit on the $B_{s}^{0} \rightarrow D_{s}^{-} \pi^{+}$decay rate. Despite the lack of a universally accepted method, it has been suggested that when setting limits in cases with Poisson distributed data and few observed events and a small expected background, the sensitivity of the experiment should be reported [1]. The sensitivity is the average upper limit which would be obtained by an ensemble of experiments with the expected background and no true signal [38]. This 
factor allows the respective experiments to be ranked by their expected results. Thus, the selection criteria for the $D_{s}$ decay modes are chosen based on the value of the sensitivity, S.

The sensitivity, $\mathrm{S}$, is defined as:

$$
S=\frac{\bar{N}\left(\mu_{B}\right)}{\epsilon\left(B_{s}\right)} \times A
$$

where $\epsilon\left(B_{s}\right)$ is the reconstruction efficiency from Monte Carlo for the $B_{s}$ decay mode and $A$ is a normalization factor. The expected background, $\mu_{B}$, is estimated from the sideband regions. The background estimates are extrapolated from the number of events in the sideband regions normalized to the $\pm 3 \sigma$ signal region. The two sideband regions extend from 4.5 to $5.3156 \mathrm{GeV} / c^{2}$ and 5.4236 to $6.0 \mathrm{GeV} / c^{2}$. The large sideband size is used to minimize fluctuations in the background. In addition, the two regions are divided into even and odd bins. The even bins are used for the optimization study while the odd bins are used for the final background fit, which will help reduce bias from low background fluctuations.

The average upper limit, $\bar{N}\left(\mu_{B}\right)$, is calculated from the number of signal events and expected background in signal region. Thus, $\bar{N}\left(\mu_{B}\right)$ is the sum of the upper limits on the number of $B_{s}$ candidates, $N\left(n, \mu_{B}\right)$, weighted by the Poisson probability, $P\left(n, \mu_{B}\right)$, to observe that number of events:

$$
\begin{gathered}
\bar{N}\left(\mu_{B}\right)=\sum_{n=0}^{\infty} P\left(n, \mu_{B}\right) \times N\left(n, \mu_{B}\right), \\
=\sum_{n=0}^{\infty} \frac{\mu_{B}^{n}}{n !} e^{-\mu_{B}} \times N\left(n, \mu_{B}\right) .
\end{gathered}
$$

The factor $A$ represents the normalization factor:

$$
A=\frac{\left(f_{d}+f_{u}\right)}{f_{s}} \times \frac{B R\left(N_{\mathrm{ref}} \times \epsilon(r e f)\right)}{B R\left(D_{s}^{i} \rightarrow X^{i}\right)} \times \frac{1}{N_{r e f}}
$$




\begin{tabular}{c|ccc} 
Variable & Min & Max & Step Size \\
\hline \hline$P_{T}\left(B_{s}\right)[\mathrm{GeV} / c]$ & 5.0 & 15.0 & 1 \\
$P_{T}\left(D_{s}\right)[\mathrm{GeV} / c]$ & 1.0 & 5.0 & .5 \\
$c \tau\left(B_{s}\right)[\mu \mathrm{m}]$ & 60 & 160 & 10 \\
$c \tau\left(D_{s}\right)[\mu \mathrm{m}]$ & -100 & 100 & 25
\end{tabular}

Table 6: The range of values and step size for each variable used in the optimization. The $B_{s}$ optimized selection criteria are found in the last column.

where $N_{\text {ref }}, \mathrm{BR}\left(N_{\text {ref }}\right)$ and $\epsilon(r e f)$ are the number of reference signal events, branching ratios and reconstruction efficiencies respectively, $f_{u, d_{,},}$is the fragmentation fraction and $\epsilon\left(B_{s}\right)$ denotes the reconstruction efficiency.

\section{3 $\quad B_{s}^{0} \rightarrow D_{s}^{-} \pi^{+}$Candidates}

To determine the selection criteria which minimize the sensitivity, the optimization variables are scanned over there possible values. The range of values for the selection criteria are listed in Table 6. This optimization procedure is applied to all $B_{s}$ decay modes. The mass of the individual $B_{s}$ candidates for each $B_{s}$ decay mode are found in the following sections. The optimization criteria for each mode and the average value of all modes is shown in Table 7. 
11.3.1 $\quad B_{s}^{0} \rightarrow D_{s}^{-} \pi^{+}, D_{s}^{-} \rightarrow \phi \pi^{-}, \phi \rightarrow K^{+} K^{-}$

For the reconstruction of $B_{s}$ candidates via the $B_{s}^{0} \rightarrow D_{s}^{-} \pi^{+}, D_{s}^{-} \rightarrow \phi \pi^{-}, \phi \rightarrow K^{+} K^{-}$mode, the mass of the $\phi$ meson is restricted to $0.979<m_{\phi}<1.059 \mathrm{GeV} / c^{2}$. After the optimization cuts are applied, the reconstruction efficiency is $0.85 \%$. The selection criteria optimized for the $B_{s}^{0} \rightarrow D_{s}^{-} \pi^{+}, D_{s}^{-} \rightarrow \phi \pi^{-}, \phi \rightarrow K^{+} K^{-}$mode are listed in Table $i$. The mass distribution of the $B_{s}^{0}$ candidates is shown in Figure 29. There are no events in the signal region and the expected background is 0.0 events. The expected sensitivity for this mode is $32 \%$ and is listed in Table 9.

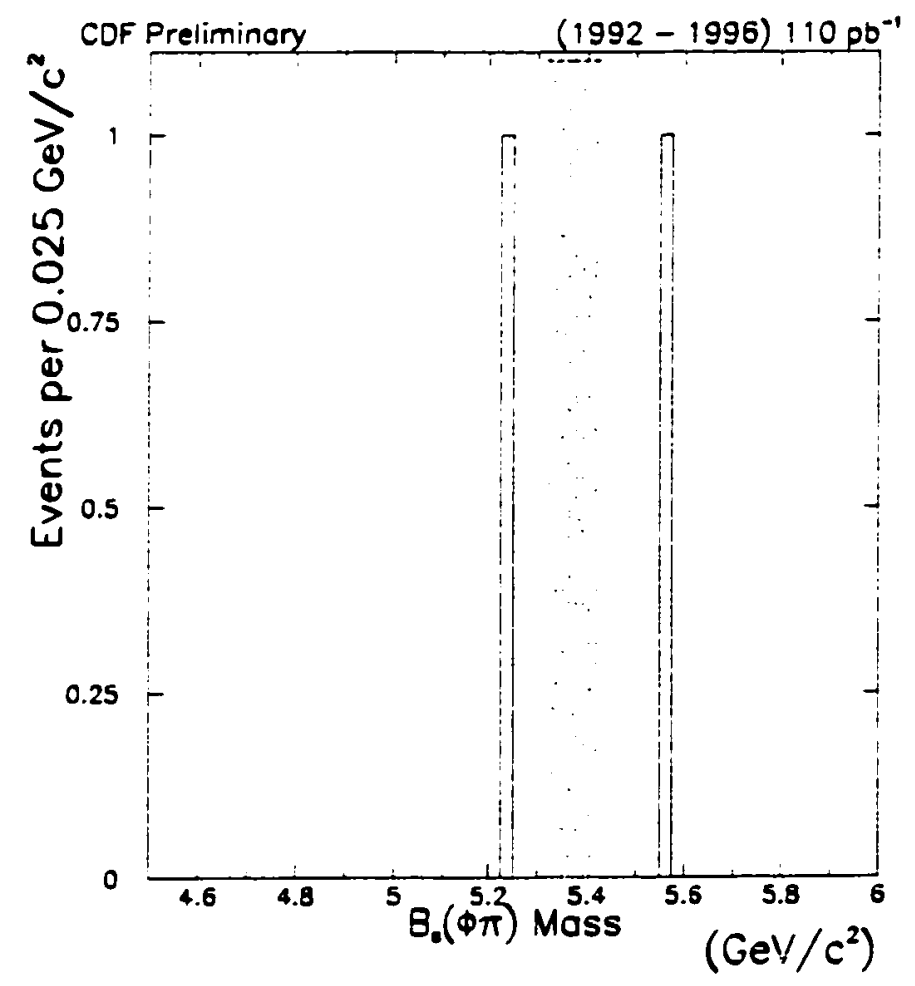

Figure 29: The mass of $B_{s}$ candidates in the $B_{s}^{0} \rightarrow D_{s}^{-} \pi^{+}, D_{s}^{-} \rightarrow \phi \pi^{-}, \phi \rightarrow K^{+} K^{-}$mode. The shaded region denotes a $\pm 3 \sigma$ region around the nominal $B_{s}$ mass. 
11.3.2 $\quad B_{s}^{0} \rightarrow D_{s}^{-} \pi^{+}, D_{s}^{-} \rightarrow \phi \pi^{-} \pi^{-} \pi^{+}, \phi \rightarrow K^{+} K^{-}$

For the reconstruction of $B_{s}$ candidates via the $B_{s}^{0} \rightarrow D_{s}^{-} \pi^{+}, D_{s}^{-} \rightarrow \phi \pi^{-} \pi^{-} \pi^{+}, \phi \rightarrow K^{+} K^{-}$ mode, the $\phi$ mass is restricted to $0.979<m_{\phi}<1.059 \mathrm{GeV} / c^{2}$. After optimization the reconstruction efficiency is $0.18 \%$. The selection criteria optimized for the $B_{s}^{0} \rightarrow D_{s}^{-} \pi^{+}, D_{s}^{-} \rightarrow$ $\phi \pi^{-} \pi^{-} \pi^{+}, \phi \rightarrow K^{+} K^{-}$mode are listed in Table 7 . The mass distribution of the $B_{s}^{0}$ candidates is shown in Figure 30. There is one event in the signal region and the expected background is 0.1 events. The expected sensitivity for this mode is $331 \%$ and is listed in Table 9.

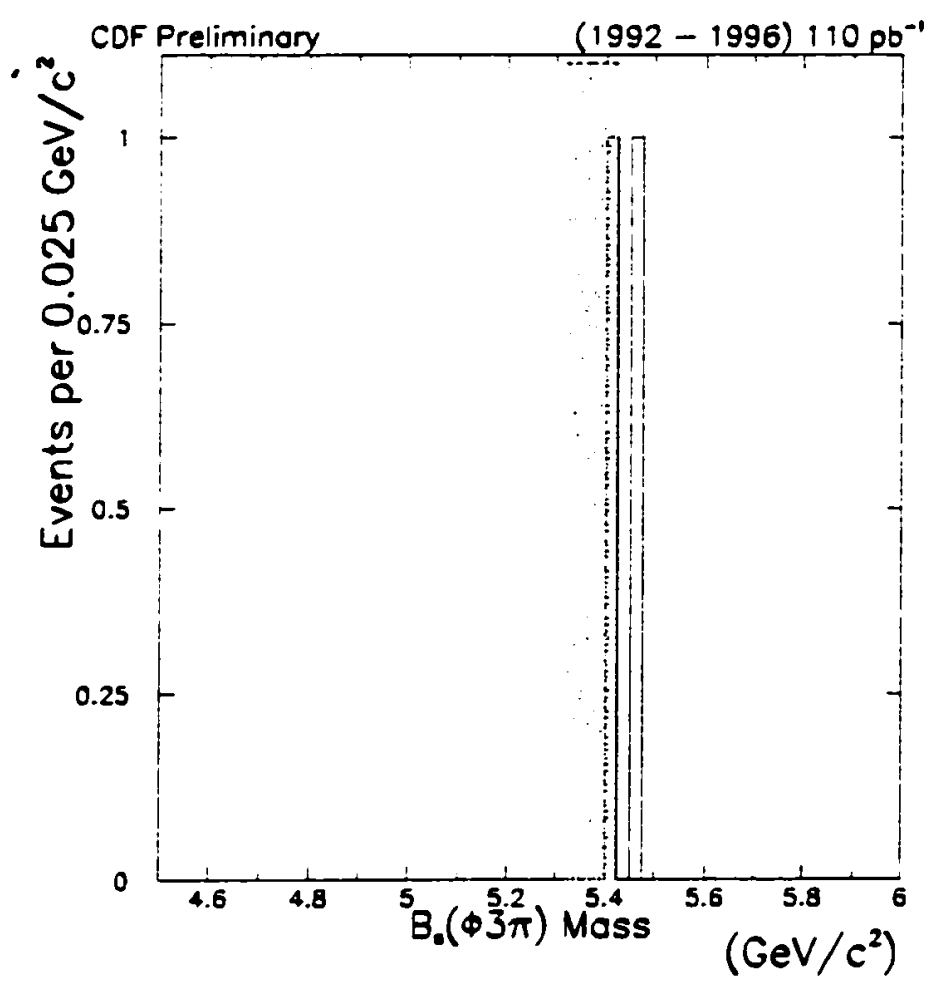

Figure 30: The mass of $B_{s}$ candidates in the $B_{s}^{0} \rightarrow D_{s}^{-} \pi^{+}, D_{s}^{-} \rightarrow \phi \pi^{-} \pi^{-} \pi^{+}, \phi \rightarrow K^{+} K^{-}$ mode. The shaded region denotes a $\pm 3 \sigma$ region around the nominal $B_{s}$ mass. 
11.3.3 $\quad B_{s}^{0} \rightarrow D_{s}^{-} \pi^{+}, D_{s}^{-} \rightarrow K^{*^{0}} K^{-}, K^{*^{0}} \rightarrow K^{+} \pi^{-}$

For the reconstruction of $B_{s}$ candidates via the $B_{s}^{0} \rightarrow D_{s}^{-} \pi^{+}, D_{s}^{-} \rightarrow K^{*^{0}} K^{-}, K^{* 0} \rightarrow K^{+} \pi^{-}$mode, the $K^{*}$ mass is restricted to $0.719<m_{K} \cdot<0.919 \mathrm{GeV} / c^{2}$. After optimization, the reconstruction efficiency is $0.91 \%$. The selection criteria optimized for the $B_{s}^{0} \rightarrow D_{s}^{-} \pi^{+}, D_{s}^{-} \rightarrow$ $K^{*^{0}} K^{-}, K^{*^{0}} \rightarrow K^{+} \pi^{-}$mode are listed in Table 7 . The mass distribution of the $B_{s}^{0}$ candidates is shown in Figure 31. There are no events in the signal region and the expected background is 1.0 event. The expected sensitivity for this mode is $24 \%$ and is listed in Table 9.

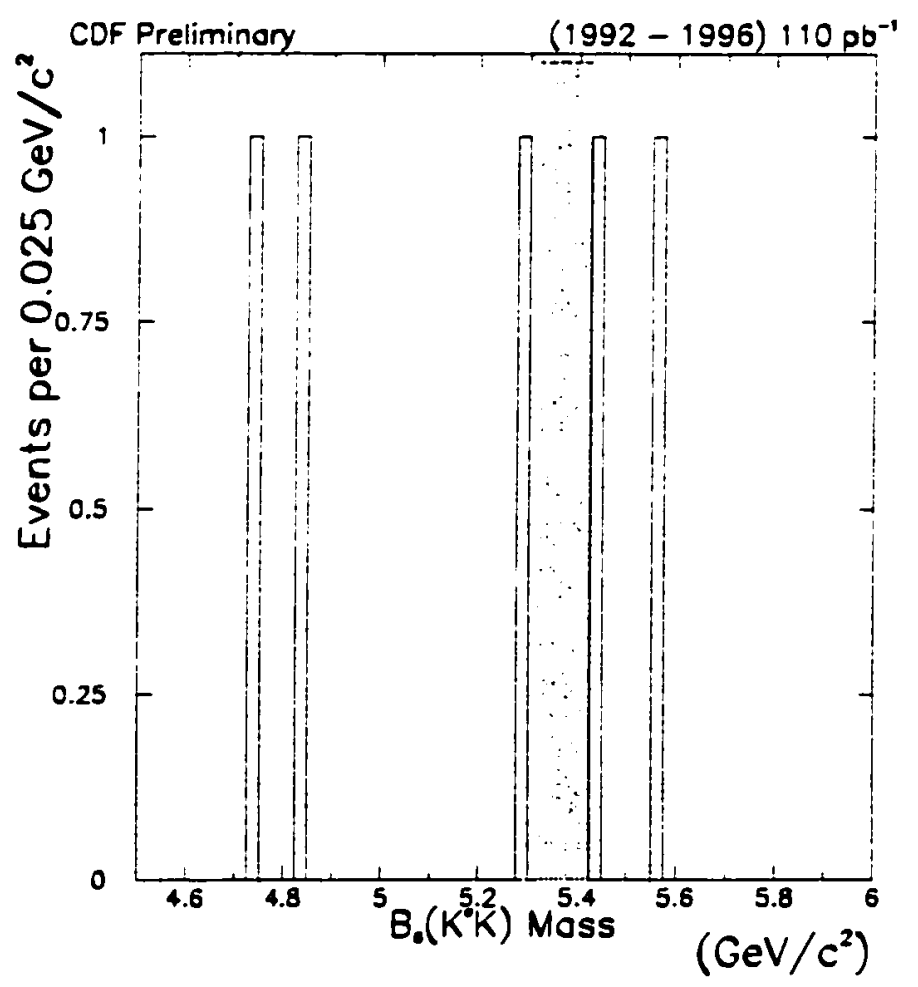

Figure 31: The mass of $B$, candidates in the $B_{s}^{0} \rightarrow D_{s}^{-} \pi^{+}, D_{s}^{-} \rightarrow K^{*^{0}} K^{-}, K^{*^{0}} \rightarrow$ $K^{+} \pi^{-}$mode. The shaded region denotes a $\pm 3 \sigma$ region around the nominal $B_{s}$ mass. 


\subsection{4 $\quad B_{s}^{0} \rightarrow D_{s}^{-} \pi^{+}, D_{s}^{-} \rightarrow K_{s} K^{-}, K_{s} \rightarrow \pi^{+} \pi^{-}$}

For the reconstruction of $B_{s}$ candidates via the $B_{s}^{0} \rightarrow D_{s}^{-} \pi^{+}, D_{s}^{-} \rightarrow K_{s} K^{-}, K_{s} \rightarrow \pi^{+} \pi^{-}$mode, the $K_{S}$ mass is restricted to $0.398<m_{K_{1}}<0.598 \mathrm{GeV} / c^{2}$ before constraining the $K_{S}$ mass in the B fit. After optimization, the reconstruction efficiency is $0.42 \%$. The selection criteria optimized for the $B_{s}^{0} \rightarrow D_{s}^{-} \pi^{+}, D_{s}^{-} \rightarrow K_{s} K^{-}, K_{s} \rightarrow \pi^{+} \pi^{-}$mode are listed in Table 7 . The mass distribution of the $B_{s}^{0}$ candidates is shown in Figure 32. There is one event in the signal region, and the expected background is 0.1 event. The expected sensitivity for this mode is $89 \%$ and is listed in Table 9.

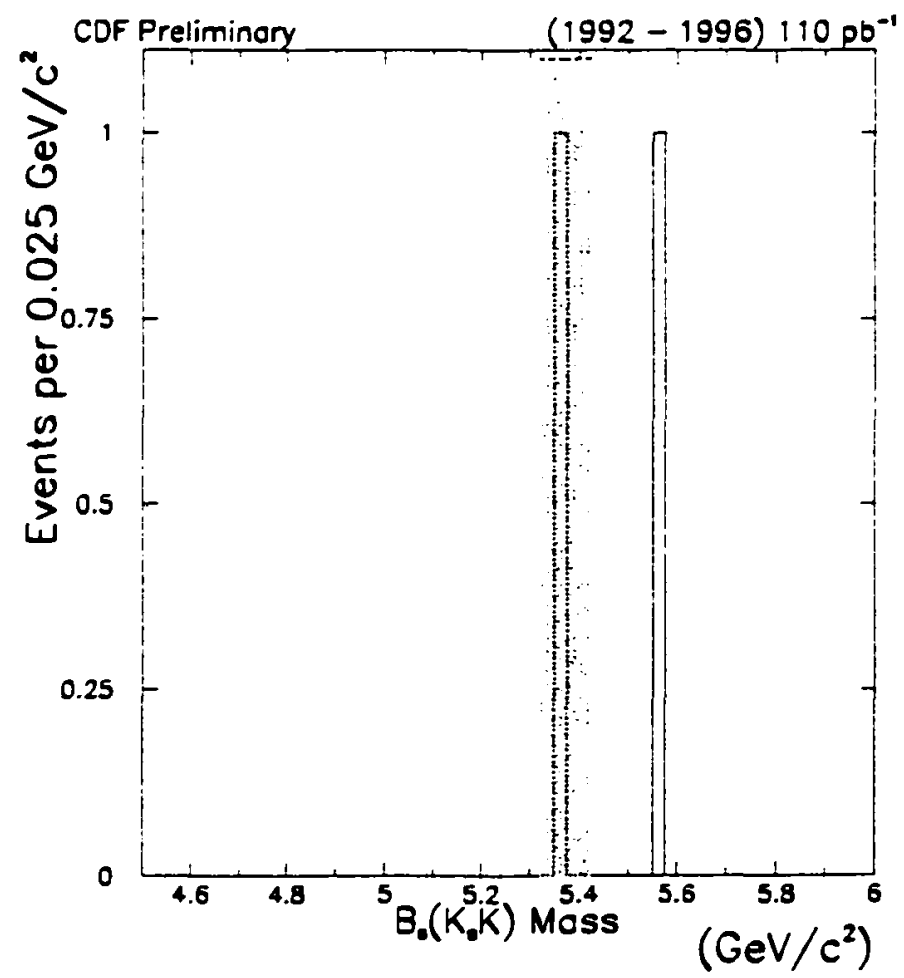

Figure 32: The mass of $B_{s}$ candidates in the $B_{s}^{0} \rightarrow D_{s}^{-} \pi^{+}, D_{s}^{-} \rightarrow K_{s} K^{-}, K_{s} \rightarrow \pi^{+} \pi^{-}$mode. The shaded region denotes a $\pm 3 \sigma$ region around the nominal $B_{s}$ mass. 
11.3.5 $\quad B_{s}^{0} \rightarrow D_{s}^{-} \pi^{+}, D_{s}^{-} \rightarrow \overline{f_{0}}(980) \pi^{-}, \overline{f_{0}}(980) \rightarrow \pi^{+} \pi^{-}$

For the reconstruction of $B_{s}$ candidates via the $B_{s}^{0} \rightarrow D_{s}^{-} \pi^{+}, D_{s}^{-} \rightarrow \overline{f_{0}}(980) \pi^{-}, \overline{f_{0}}(980) \rightarrow$ $\pi^{+} \pi^{-}$mode, the $f^{0}$ mass is restricted to $0.88<m_{f^{0}}<1.08 \mathrm{GeV} / c^{2}$. After optimization, the reconstruction efficiency is $0.79 \%$. The selection criteria optimized for the $B_{s}^{0} \rightarrow$ $D_{s}^{-} \pi^{+}, D_{s}^{-} \rightarrow \overline{f_{0}}(980) \pi^{-}, \overline{f_{0}}(980) \rightarrow \pi^{+} \pi^{-}$mode are listed in Table 7 . The mass distribution of the $B_{s}^{0}$ candidates is shown in Figure 33. There are no events in the signal region, and the expected background is 0.6 events. The expected sensitivity for this mode is $46 \%$ and is listed Table 9.

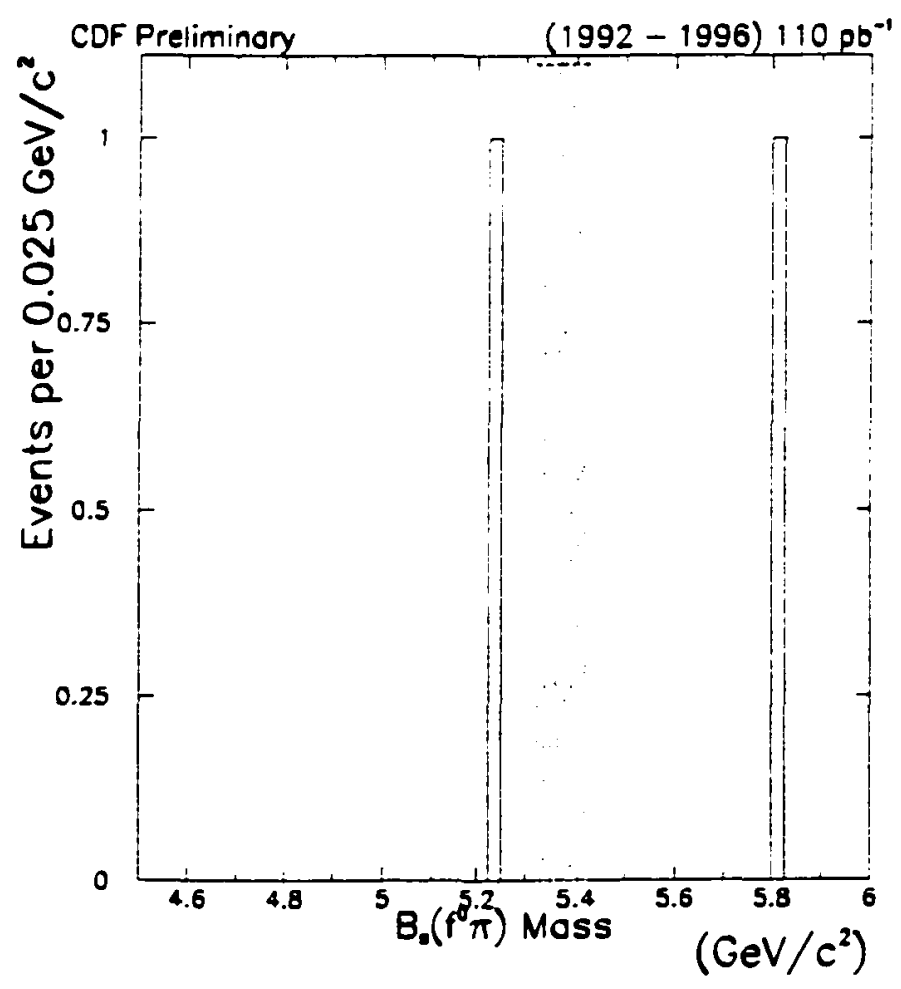

Figure 33: The mass of $B_{s}$ candidates in the $B_{s}^{0} \rightarrow D_{s}^{-} \pi^{+}, D_{s}^{-} \rightarrow \overline{f_{0}}(980) \pi^{-}, \overline{f_{0}}(980) \rightarrow \pi^{+} \pi^{-}$ mode. The shaded region denotes a $\pm 3 \sigma$ region around the nominal $B_{s}$ mass. 


\subsection{6 $\quad B_{s}^{0} \rightarrow D_{s}^{-} \pi^{+}, D_{s}^{-} \rightarrow K^{0^{0}} \pi^{-}, K^{0^{0}} \rightarrow K^{+} \pi^{-}$}

For the reconstruction of $B_{s}$ candidates via the $B_{s}^{0} \rightarrow D_{s}^{-} \pi^{+}, D_{s}^{-} \rightarrow K^{\bullet^{0}} \pi^{-}, K^{\bullet^{0}} \rightarrow K^{+} \pi^{-}$mode, the $K^{*}$ mass is restricted to $0.719<m_{K^{*}}<0.919 \mathrm{GeV} / c^{2}$. After optimization the reconstruction efficiency is $0.72 \%$. The selection criteria optimized for the $B_{s}^{0} \rightarrow D_{s}^{-} \pi^{+}, D_{s}^{-} \rightarrow$ $K^{*^{0}} \pi^{-}, K^{*^{0}} \rightarrow K^{+} \pi^{-}$mode are listed in Table 7. The mass distribution of the $B_{s}^{0}$ candidates is shown in Figure 34. There is one event in the signal region and the expected background is 0.3 events. The expected sensitivity for this mode is $119 \%$ and is listed in Table 9 .

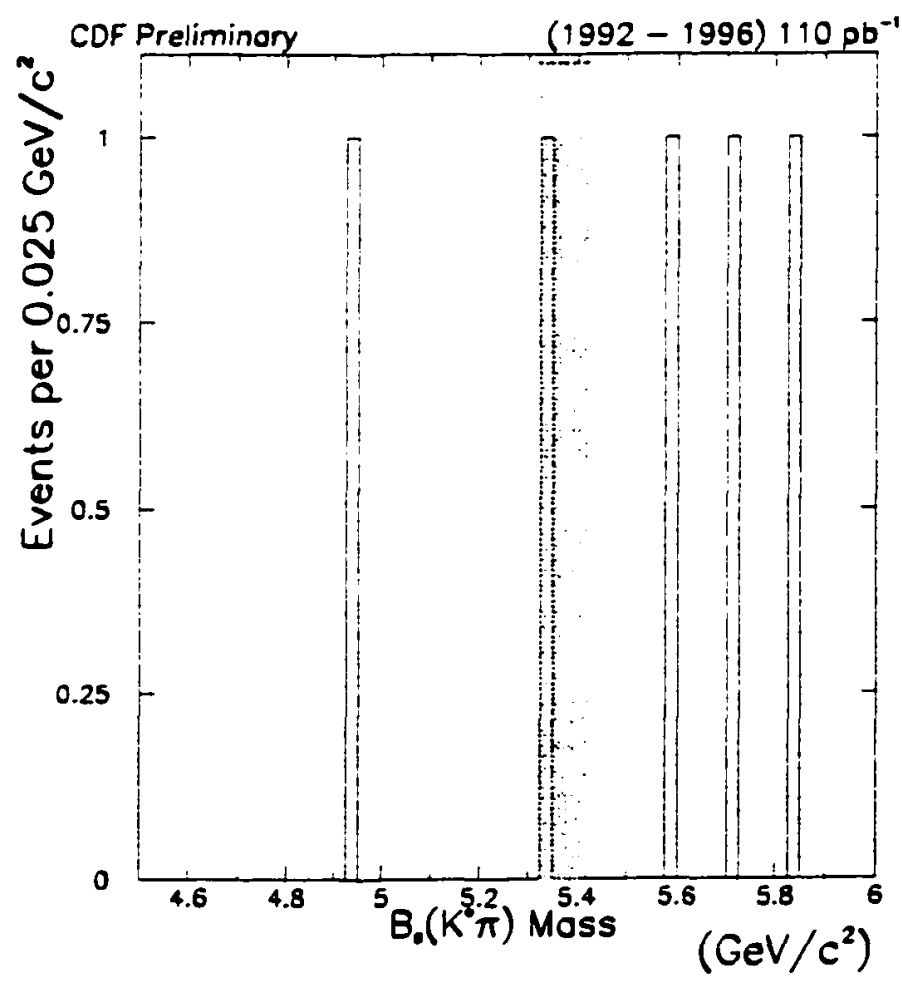

Figure 34: The mass of $B_{s}$ candidates in the $B_{s}^{0} \rightarrow D_{s}^{-} \pi^{+}, D_{s}^{-} \rightarrow K^{*^{0}} \pi^{-}, K^{*^{0}} \rightarrow K^{+} \pi^{-}$mode. The shaded region denotes a $\pm 3 \sigma$ region around the nominal $B_{s}$ mass. 

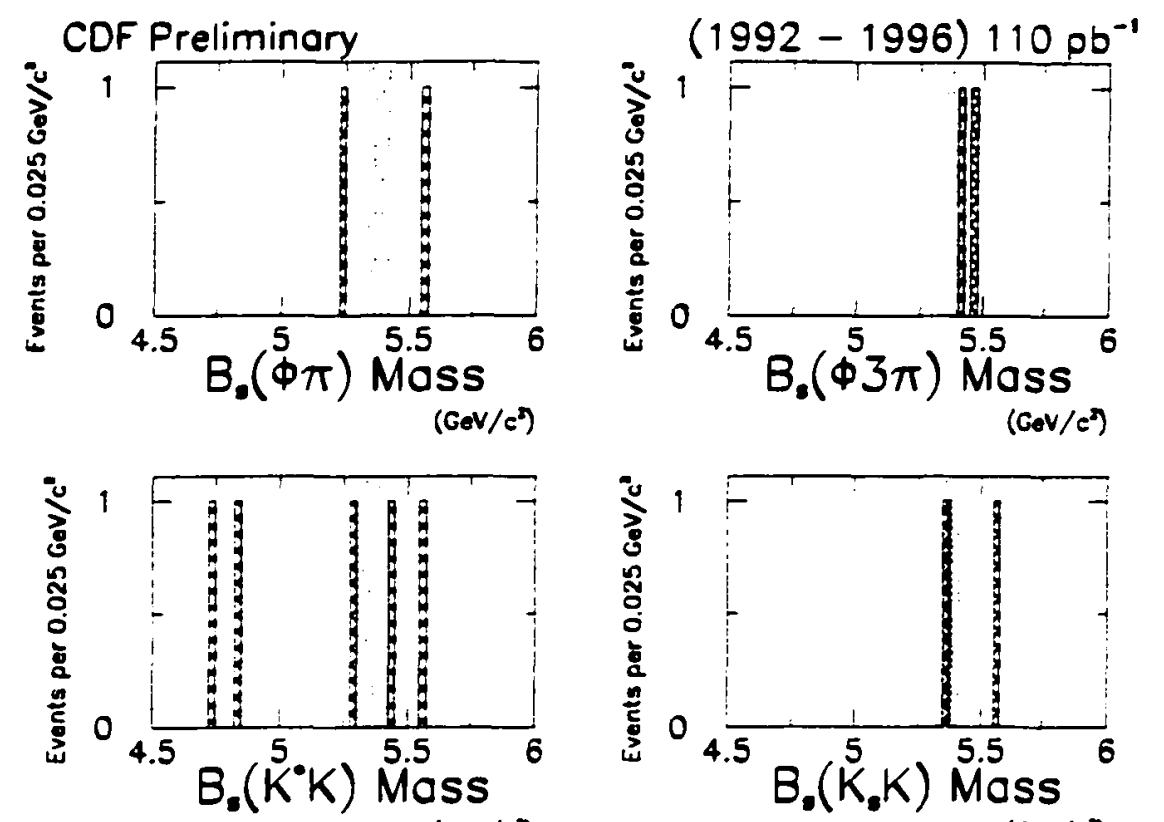

$\left(\mathrm{GeV} / \mathrm{c}^{2}\right)$

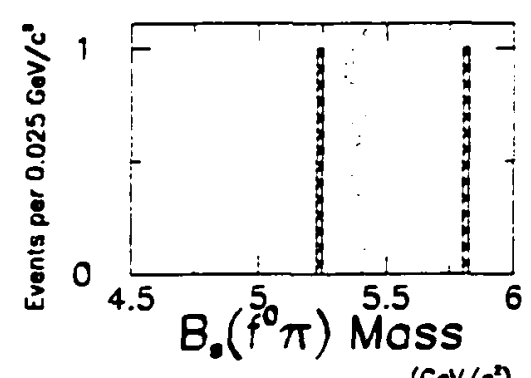

$\left(\mathrm{GeV} / \mathrm{c}^{2}\right)$

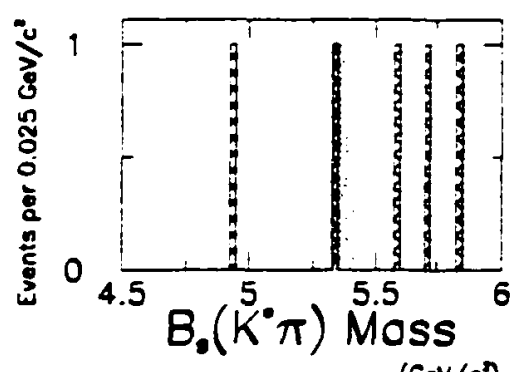

(Gov/e')

Figure 35: The six plot shown above are the six $B_{s}^{0} \rightarrow D_{s}^{-} \pi^{+}$decay modes. The shaded region denotes a $\pm 3 \sigma$ region around the nominal $B_{s}$ mass.

\subsection{Combined $B_{s}$ Mass}

In the mass distribution of the combined $B_{s}$ candidates for all decay modes, there are three candidate events in the signal region with an expected background of 0.825 events. The candidates in each mode after the optimized cuts are applied are shown in Figure 35. The combined mass plot is shown 


\begin{tabular}{c|cccc} 
MODE & $P_{T}\left(B_{s}\right)[\mathrm{GeV} / c]$ & $P_{T}\left(D_{s}\right)[\mathrm{GeV} / c]$ & $c \tau\left(B_{s}\right)[\mu \mathrm{m}]$ & $c \tau\left(D_{s}\right)[\mu \mathrm{m}]$ \\
\hline$\phi \pi^{-}$ & 8.0 & 3.0 & 90 & -50 \\
$\phi \pi^{+} \pi^{-} \pi^{-}$ & 8.0 & 2.0 & 90 & 25 \\
$K^{* 0} K^{-}$ & 11.0 & 2.0 & 90 & -25 \\
$K_{s} K^{-}$ & 8.0 & 2.0 & 100 & -50 \\
$\overline{f^{0}} \pi^{-}$ & 9.0 & 2.0 & 100 & -25 \\
$K^{* 0} \pi^{-}$ & 10.0 & 2.0 & 120 & -75 \\
\hline \hline AVERAGE & 9.0 & 2.0 & 100 & -25
\end{tabular}

Table 7: The optimization cuts for each $B_{s} \rightarrow D_{s} \pi$ decay mode. The $D_{s} \pi$ decay mode modes are identified in the first column. The last row contains the average values.

in Figure 36. All of the candidates events found in the signal region are assumed to be signal events.

\subsection{Average Selection}

Since the cuts are all very similar and are most likely driven by limited statistics, the average value of the cuts is used. The candidates in each mode after the average values are applied to each mode is shown in Figure 37 and the upper limit on the number of events in the signal region is determined from the combined mass plot. The $B_{s}$ candidates are combined into into a final mass plot shown in Figure 38. The combined mass plot has two signal event with an expected background of 0.77 events. 


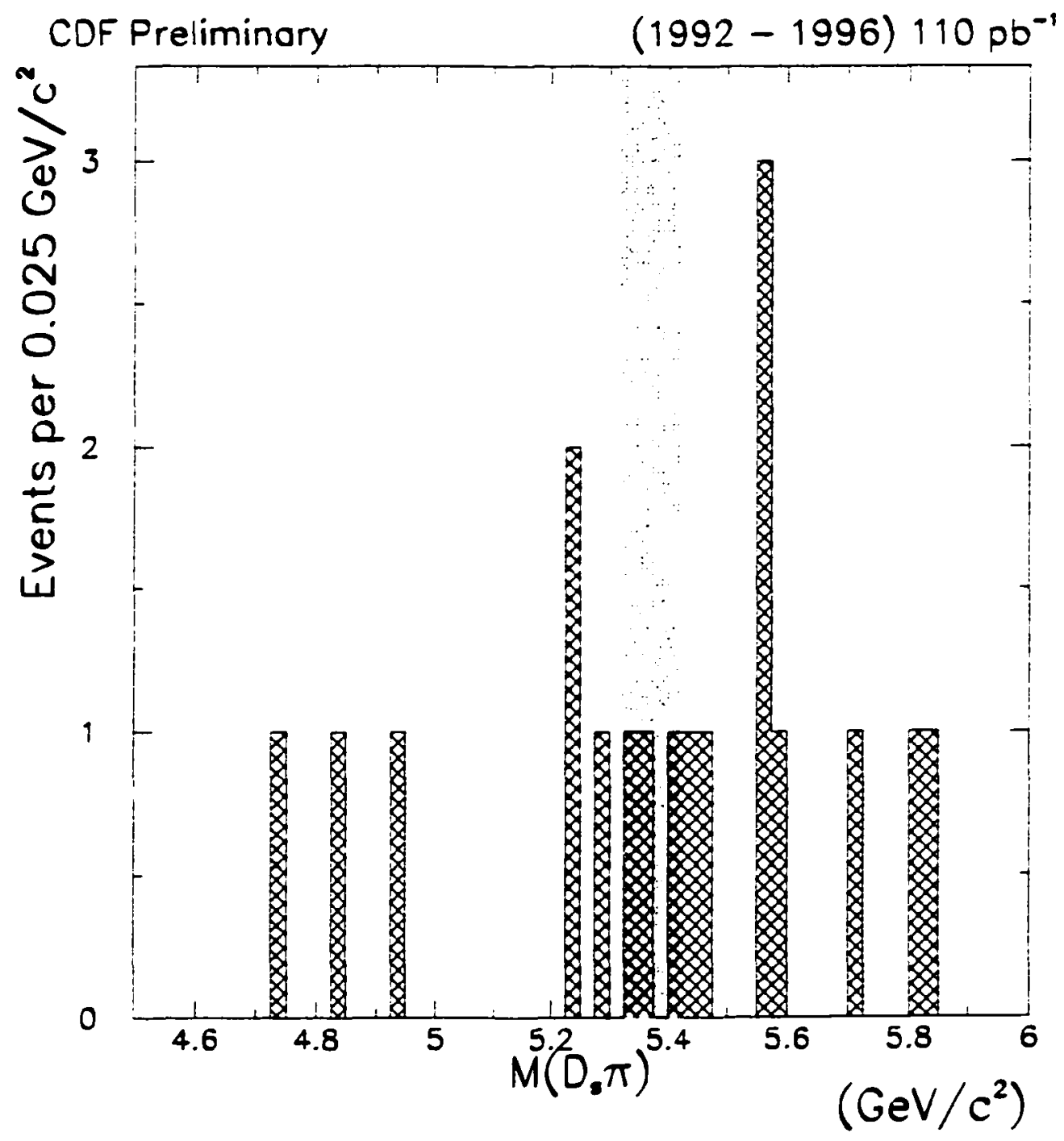

Figure 36: The combined mass plot for all $B_{s}^{0} \rightarrow D_{s}^{-} \pi^{+}$decay modes. The shaded region is the $\pm 3 \sigma$ signal mass region. 

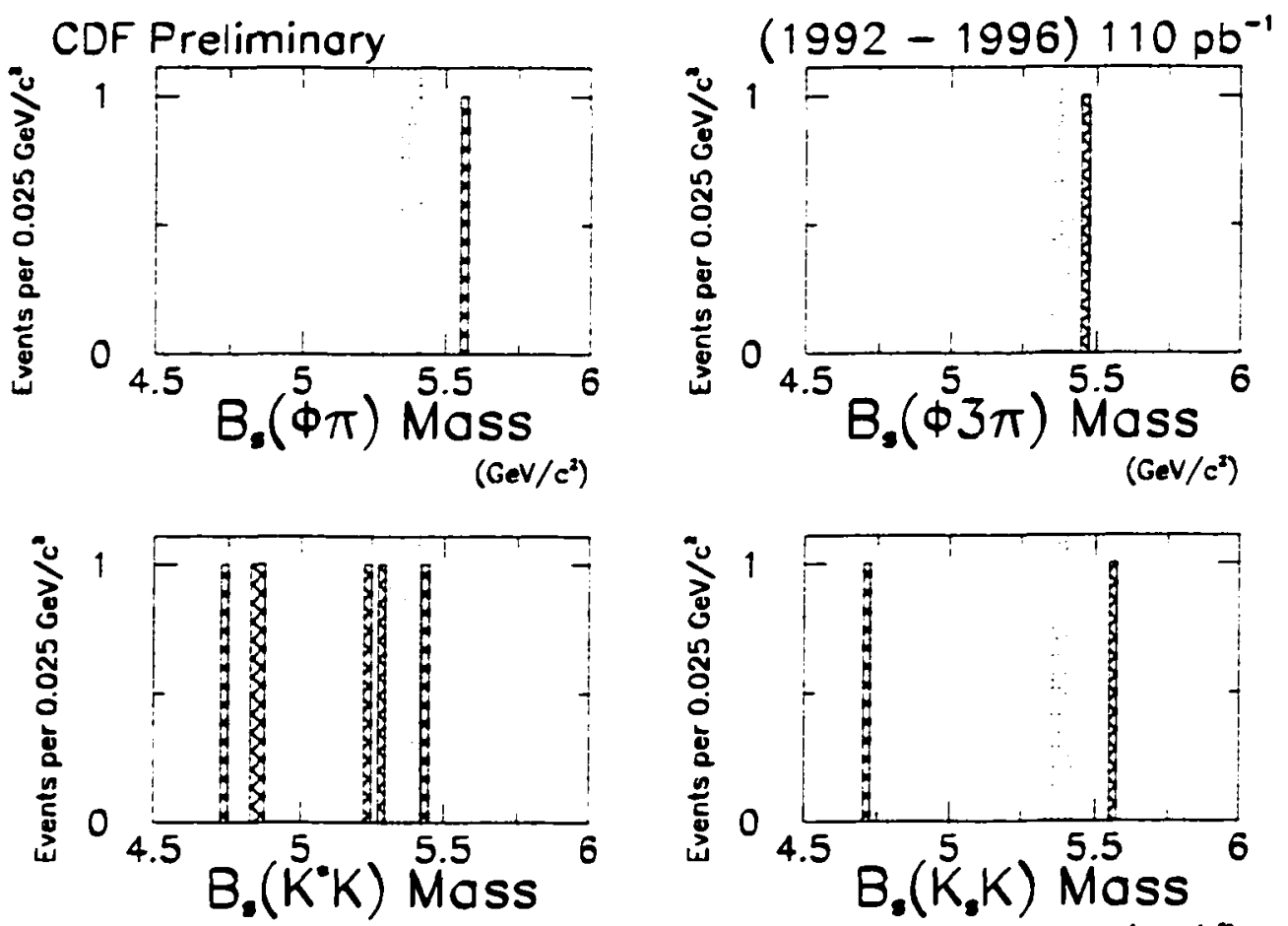

$\left(\mathrm{GeV} / \mathrm{c}^{2}\right)$
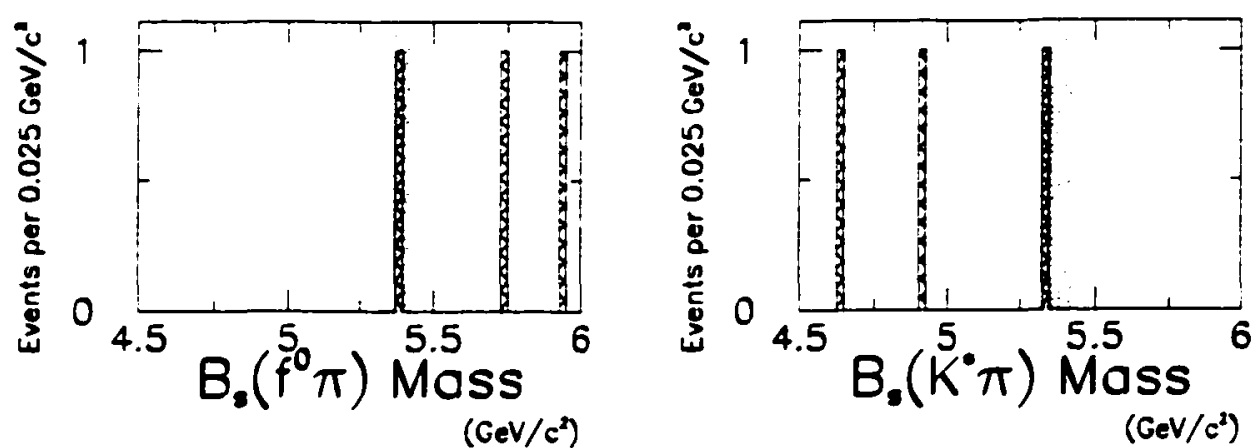

Figure 37: The mass plot for all $B_{s}^{0} \rightarrow D_{s}^{-} \pi^{+}$decay modes with the AVERAGE selection criteria. The shaded region is the $\pm 3 \sigma$ signal mass region. 


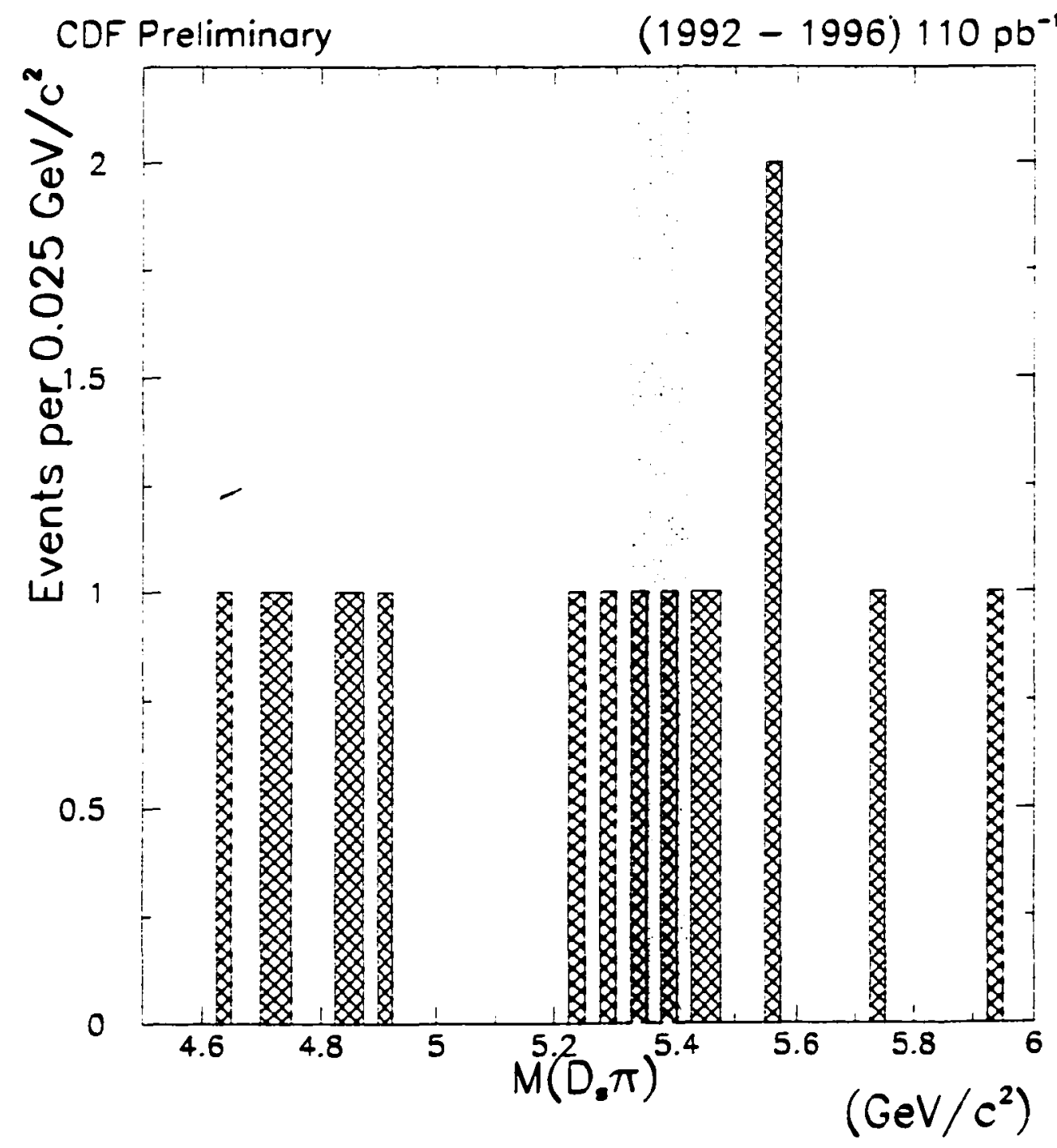

Figure 38: The combined mass plot for all $B_{s}^{0} \rightarrow D_{s}^{-} \pi^{+}$decay modes with the AVERAGE selection criteria. The shaded region is the $\pm 3 \sigma$ signal mass region. 


\section{Branching Ratio Limit}

An upper limit on the $B_{s}^{0} \rightarrow D_{s}^{-} \pi^{+}$branching ratio is a product of the upper limit on the number of events in $B_{S}$ signal region, the number of $B^{+}$and $B^{0}$ events in the reference signal, and a scaling factor. The scaling factor includes the fragmentation fractions, branching ratios, and efficiencies used in the branching ratio calculation. For a direct measurement of the branching fraction, the efficiencies and absolute geometric and kinematic acceptance, trigger, reconstruction and final selection cuts must be known. However, in a relative measurement some of these factors cancel and equally importantly the uncertainty on these values cancel. The dominate statistical and systematic uncertainties are presented in Section 12.1. The methods used to choose which decay modes to include in our branching ratio calculation is presented in Section 12.2. The calculation of the upper limit on the $B_{s}^{0} \rightarrow D_{s}^{-} \pi^{+}$branching ratio is detailed in Section 12.3.

\subsection{Uncertainties}

Based on the number of events in the signal region and the expected background, an upper limit on the number of events in the $B_{s}$ signal region. However, to convert the upper limit on the number of events to an upper limit is placed on a branching fraction the uncertainties in the scaling factor must be included. The number of events in the reference signal has an uncertainty associated with it. The Gaussian term in the likelihood function returns an uncertainty on the number of signal events. Also, the product branching ratios for the $D$, decay modes have significant uncertainties as well as correlated uncertainties between the $D_{s}$ decay modes[1;. In addition there are uncertainties on the fragmentation fractions. A $2 \%$ inefficiency for each track from a $K_{s}$ is assigned. These uncertainties are added in quadrature and are found in Table 8. 


\begin{tabular}{|c|c|}
\hline \hline Systematic Uncertainty & $\%$ \\
\hline Reference Signal & 50 \\
BR $\times$ Efficiency & 26 \\
$f_{s} /\left(f_{d}+f_{u}\right)$ & 18 \\
$K_{s}$ Track Efficiency & 4 \\
\hline Combined Systematics & 59 \\
\hline \hline
\end{tabular}

Table 8: Each of the systematic uncertainties in the components of the scaling factor are listed as well as the total systematic error.

\subsection{Limit Studies}

In this section, the ability of the six $B_{s}^{0} \rightarrow D_{s}^{-} \pi^{+}$decay modes to set an upper limit on the $B_{s}^{0} \rightarrow$ $D_{s}^{-} \pi^{+}$branching ratio is studied. Because the expected background between various decay mode and the reconstruction efficiency vary, the sensitivity of each mode will vary. In addition, different combinations of modes will have different sensitivities. These issues are explored in the following sections. Based on the relative value of these sensitivities, the $B_{3}$ modes to include are chosen based on their ability to achieve the best sensitivity.

\subsubsection{Comparison of Modes}

Each of the $B_{s}^{0} \rightarrow D_{s}^{-} \pi^{+}$decay modes are evaluated individually. For each $B_{s}^{0} \rightarrow D_{s}^{-} \pi^{+}$decay mode, the product of the branching ratio and reconstruction efficiency, the expected background and sensitivity in each mode is shown in Table 9. This table indicates that the $\phi \pi$ and $K^{*} K$ modes offer the best sensitivity while the $\phi \pi \pi \pi$ modes has the worst sensitivity. 


\begin{tabular}{|c|c|c|c|c|}
\hline \hline Index & $D_{s}$. Mode & $B R \times \epsilon\left(\times 10^{-4}\right)$ & Expected Background & Sensitivity (\%) \\
\hline 1 & $\phi \pi$ & 1.3 & 0.0 & 32 \\
\hline 2 & $\phi \pi \pi \pi$ & 0.1 & 0.3 & 331 \\
\hline 3 & $K^{*} \mathrm{~K}$ & 1.9 & 0.3 & 24 \\
\hline 4 & $K_{s} \mathrm{~K}$ & 0.5 & 0.0 & 89 \\
\hline 5 & $f^{0} \pi$ & 0.9 & 0.0 & 46 \\
\hline 6 & $K^{*} \pi$ & 0.4 & 0.1 & 119 \\
\hline \hline
\end{tabular}

Table 9: The sensitivity of each $B_{s} \rightarrow D_{s} \pi$ decay mode is found in the last column. The $D_{s}$ modes are listed in the second column.

\subsubsection{Combination of Modes}

The sensitivity of different combinations of $B_{s}^{0} \rightarrow D_{s}^{-} \pi^{+}$decay modes is calculated. The combination chosen will be the one which has the smallest expected sensitivity. The difference in sensitivity for different combinations is not expected to be large. The expected sensitivity for different combinations of $B_{s}$ decay modes is shown in Table 10 . The greatest sensitivity, $9.6 \%$ is achieved when all decay modes, except the $K^{*} \pi$ mode, are included. However, due to the uncertainty associated with the combined sensitivity values, all $B_{\mathrm{s}}$ decay modes are included in the limit calculation. The combination of all $B_{s}$ modes has a sensitivity of $10.2 \%$. 


\begin{tabular}{|c|c|c|c|}
\hline \hline Modes & $B R \times \epsilon\left(\times 10^{-4}\right)$ & Expected Background & Sensitivity (\%) \\
\hline \hline $1,3,4,5,6$ & 5.0 & 0.4 & 9.6 \\
\hline $1,3,4,5$ & 4.6 & 0.3 & 10.1 \\
\hline ALL & 5.1 & 0.8 & 10.2 \\
\hline $1,3,5,6$ & 4.5 & 0.4 & 10.6 \\
\hline $1,2,3,4,5$ & 4.7 & 0.7 & 10.7 \\
\hline $1,3,5$ & 4.1 & 0.3 & 11.2 \\
\hline \hline
\end{tabular}

Table 10: The sensitivity for different combination of $B_{s} \rightarrow D_{s} \pi$ decay modes.

\subsubsection{Optimal Signal Region}

The optimal width of the signal region around the nominal $B_{s}$ mass is studied based on the expected background and the product of the branching ratio and reconstruction efficiency. To simplify the study only $\pm 2 \sigma$ and $\pm 3 \sigma$ regions are studied. The one sigma width is equal to the ratio of the $B_{s}$ and $B^{+}, B^{0}$. Wonte Carlo widths and the average uncertainty of $B^{+}, B^{0}$ candidates used in the reference signal $\left(18 \mathrm{MeV} / c^{2}\right)$. The sensitivity for each region is listed in Table 11. The signal region defined as $\pm 3 \sigma$ around the nominal $B$, mass offer slightly better resolution. 


\begin{tabular}{|c|c|c|c|}
\hline Modes & $\begin{array}{c}B R \times \epsilon \\
{\left[10^{-4}\right]}\end{array}$ & $\begin{array}{l}\text { Expected } \\
\text { Background }\end{array}$ & $\begin{array}{l}S \\
\%\end{array}$ \\
\hline 2 Sigma & 4.4 & 0.51 & 11.2 \\
\hline 3 Sigma & 5.1 & 0.77 & 10.2 \\
\hline
\end{tabular}

Table 11: The sensitivity for two definitions of the signal region around the $B_{s}$ mass. Top top row lists the sensitivity for a $\pm 2 \sigma$ window and the bottom row lists the sensitivity for a $\pm 3 \sigma$ window.

\section{3 $B_{s}^{0} \rightarrow D_{s}^{-} \pi^{+}$Branching Ratio Upper Limit}

The branching ratio for $B_{s}^{0} \rightarrow D_{s}^{-} \pi^{+}$decay rate can be calculated in a variety of methods. Since this is a relative measurement many uncertainties cancel. The upper limit on the $B_{s}^{0} \rightarrow D_{s}^{-} \pi^{+}$branching ratio can be calculated from the following equation:

$B R\left(B_{s} \rightarrow D_{s} \pi\right)=\frac{N\left(B_{s}\right)}{N\left(B_{d}\right)+N\left(B_{u}\right)} \times \frac{\left(f_{u}+f_{d}\right)}{f_{s}} \times \frac{\left(B R\left(B_{d} \rightarrow D \pi\right) \times \epsilon^{B_{d}}+B R\left(B_{u} \rightarrow D \pi\right) \times \epsilon^{B_{u}}\right)}{\sum_{i=1}^{6} B R\left(D_{s} \rightarrow X^{i}\right) \times \epsilon^{i}}$,

where $N\left(B_{s}\right)$ is the upper limit on the number of $B_{s}$ candidates.

Currently there is no consensus in the physics community for setting confidence limits when there are large uncertainties or when the number of signal events is small. Due to uncertainties on the number of events in the reference signal, the branching ratios, fragmentation factors and reconstruction efficiencies the calculation method varies. Two methods for calculating the upper limit on the number of signal events in our $B_{s}^{0} \rightarrow D_{s}^{-} \pi^{+}$reconstruction are presented. 


\subsubsection{Upper Limit Without Uncertainties}

The upper limit on the $\mathrm{BR}\left(B_{s} \rightarrow D_{s} \pi\right)$ branching ratio without considering the systematic and statistical uncertainties are presented in this section. The upper limits are calculated for both the average selection criteria and the individual decay modes optimized criteria. The method suggested by References[1] [38] corrects for the overcoverage of the frequentiest method for small Poisson signals. This method uses an ordering principle based on the ratio of likelihoods when choosing values of the upper limit, $n$, to place in the acceptance interval [38]. By simply using the number of event in the signal region, two, after the average selection criteria, and the expected background, 0.77, the Feldman and Cousins approach gives confidence intervals of $[0.0,4.4]$ and $[0.0,5.4]$ events at $90 \%$ and $95 \%$ C.L., respectively. Using the upper limit of these confidence intervals, the upper limit on the $\operatorname{BR}\left(B_{s} \rightarrow D_{s} \pi\right)$ branching ratio are found to be:

$$
\begin{aligned}
& B R\left(B_{s} \rightarrow D_{s} \pi\right)<5.9 \% \text { at } 90 \% \text { C.L. } \\
& B R\left(B_{s} \rightarrow D_{s} \pi\right)<7.2 \% \text { at } 95 \% \text { C.L. }
\end{aligned}
$$

and

$$
\begin{aligned}
& f\left(b \rightarrow B_{s}\right) \times B R\left(B_{s} \rightarrow D_{s} \pi\right)<0.9 \% \text { at } 90 \% \text { C.L. } \\
& f\left(b \rightarrow B_{s}\right) \times B R\left(B_{s} \rightarrow D_{s} \pi\right)<1.2 \% \text { at } 95 \% \text { C.L. }
\end{aligned}
$$

By simply using the number of event in the signal region, three, after the optimimized selection criteria for each mode, and the expected background, 0.825, the Feldman and Cousins approach gives confidence intervals of $[0.0,5.8]$ and $[0.0,6.9]$ events at $90 \%$ and $95 \%$ 
C.L., respectively. Using the upper limit of these confidence intervals, the upper limit on the $\operatorname{BR}\left(B_{s} \rightarrow D_{s} \pi\right)$ branching ratio are found to be:

$$
\begin{aligned}
& B R\left(B_{s} \rightarrow D_{s} \pi\right)<7.7 \% \text { at } 90 \% \text { C.L. } \\
& B R\left(B_{s} \rightarrow D_{s} \pi\right)<9.2 \% \text { at } 95 \% \text { C.L. }
\end{aligned}
$$

and

$$
\begin{aligned}
& f\left(b \rightarrow B_{s}\right) \times B R\left(B_{s} \rightarrow D_{s} \pi\right)<1.2 \% \text { at } 90 \% \text { C.L. } \\
& f\left(b \rightarrow B_{s}\right) \times B R\left(B_{s} \rightarrow D_{s} \pi\right)<1.5 \% \text { at } 95 \% \text { C.L. }
\end{aligned}
$$

\subsubsection{Upper Limit with Systematic and Background Uncertainties}

Since the amount of background in the combined $B_{s}^{0} \rightarrow D_{s}^{-} \pi^{+}$mass plot is significant the uncertainty in the background in the upper limit as well as the systematic uncertainty must be considered. The uncertainty in the expected background is found in Table 12. The upper

\begin{tabular}{|l|l|}
\hline \hline Systematic Uncertainty & $\%$ \\
\hline Expected Background & 30 \\
\hline
\end{tabular}

Table 12: This table shows the background uncertainty extrapolated from fit of background to signal region.

limit, $N$, on the number of expected events is defined as the value of $\mu$ for which there is some probability $\epsilon$ to observe $n_{0}$ or fewer events [39]. The confidence interval is $1-\epsilon$ where:

$$
\epsilon=\sum_{n=0}^{n_{0}} P(n \mid \mu)
$$


and

$$
P\left(n_{0} \mid \mu\right)=\frac{\mu^{n_{0}} e^{-\mu}}{n_{0} !}
$$

In most experiments, there are background events in the signal region in addition to the signal events. If one knows $\mu_{B}$ precisely, then the above method can be extended to calculate the Poisson upper limit $N$ on the number of signal events [40]:

$$
1-\epsilon=\frac{\sum_{n=0}^{n_{0}} P\left(n \mid \mu_{B}+N\right)}{\sum_{n=0}^{n_{o}} P\left(n \mid \mu_{B}\right)} .
$$

The method suggested by Reference [39] is a Bayesian-style integration over the uncertainties in the signal acceptance $\left(\sigma_{A}\right)$ and expected background $\left(\mu_{B}\right)$ :

$$
\epsilon=\frac{\sum_{n=0}^{n 0} \frac{1}{\sqrt{2 \pi \sigma_{v}^{2}}} \int_{0}^{\infty} \int_{0}^{\infty} P\left(n: \mu_{B}^{\prime}+\mu_{S}^{\prime}\right) e^{\frac{-\left(\mu_{B}-\mu_{B}^{\prime}\right)^{2}}{2 \sigma_{B}^{2}}} e^{\frac{-\left(N-\mu_{\mu}^{\prime}\right)^{2}}{2 \sigma_{V}^{2}}} d_{\mu_{B}}^{\prime} d_{\mu_{S}}^{\prime}}{\sum_{n=0}^{n_{0}} \int_{0}^{\infty} P\left(n ; \mu_{B}\right) e^{\frac{-\left(\mu_{B}-\mu_{B}^{\prime}\right)^{2}}{2 \sigma_{B}^{2}}} d_{\mu_{B}}^{\prime}} .
$$

Where $\sigma_{N}=\frac{N \sigma_{A}}{A}$ where $A$ is the overall acceptance, $N$ is the Poisson upper limit $\mu_{s}=\frac{\sigma_{A}}{A}$ and $\mu_{b}$ is the expected background. A Monte Carlo method is implemented in a FORTR.A.V program poilim.f. A large number of pseudoexperiments are generated in which the expected signal and background are varied within their uncertainties. For each experiment an expected number of signal and background events are chosen from Gaussian distributions to generate a Poisson distributed number of signal $\left(n_{s}\right)$ and background $\left(\mu_{B}\right)$ events. For the trials in which $n_{B} \leq n_{o}$, the fraction $f$ in which $n_{B}+n_{s}>n_{0}$ is recorded. The confidence level for a given $N$ is the value of $f$. The desired confidence level is achieved by varying $N$ until the desired confidence level $(1-\epsilon)$ is achieved.

If the uncertainties in the background, 0.30 events, are included and the systematic uncertainties, $59 \%$, the confidence interval after applying the average selection criteria, is 
$[0.0,7.4]$ and $[0.0,11.9]$ at $90 \%$ and $95 \%$ C.L. Inserting the upper limit of these confidence interval, the upper limit on the $\mathrm{BR}\left(B_{s} \rightarrow D_{s} \pi\right)$ branching ratio is found to be:

$$
\begin{aligned}
& B R\left(B_{s} \rightarrow D_{s} \pi\right)<9.9 \% \text { at } 90 \% \text { C.L. } \\
& B R\left(B_{s} \rightarrow D_{s} \pi\right)<15.9 \% \text { at } 95 \% \text { C.L. }
\end{aligned}
$$

and

$$
\begin{aligned}
& f\left(b \rightarrow B_{s}\right) \times B R\left(B_{s} \rightarrow D_{s} \pi\right)<1.6 \% \text { at } 90 \% \text { C.L. } \\
& f\left(b \rightarrow B_{s}\right) \times B R\left(B_{s} \rightarrow D_{s} \pi\right)<2.6 \% \text { at } 95 \% \text { C.L. }
\end{aligned}
$$

If the uncertainties in the background, 0.31 events, are included and the systematic uncertainties, $59 \%$, the confidence interval after applying the individually optimized selection criteria, is $[0.0,9.8]$ and $[0.0,16.0]$ at $90 \%$ and $95 \%$ C.L. Inserting the upper limit of these confidence interval, the upper limit on the $\mathrm{BR}\left(B_{s} \rightarrow D_{s} \pi\right)$ branching ratio is found to be:

$$
\begin{aligned}
& B R\left(B_{s} \rightarrow D_{s} \pi\right)<13.0 \% \text { at } 90 \% \text { C.L. } \\
& B R\left(B_{s} \rightarrow D_{s} \pi\right)<21.3 \% \text { at } 95 \% \text { C.L. }
\end{aligned}
$$

and

$$
\begin{aligned}
& f\left(b \rightarrow B_{s}\right) \times B R\left(B_{s} \rightarrow D_{s} \pi\right)<2.1 \% \text { at } 90 \% \text { C.L. } \\
& f\left(b \rightarrow B_{s}\right) \times B R\left(B_{s} \rightarrow D_{s} \pi\right)<3.5 \% \text { at } 95 \% \text { C.L. }
\end{aligned}
$$




\section{Summary}

We have reported an upper limit on $B_{s} \rightarrow D_{s} \pi$ decay. The results of this thesis are summarized as:

$$
\begin{aligned}
& B R\left(B_{s} \rightarrow D_{s} \pi\right)<9.9 \% \text { at } 90 \% \text { C.L. } \\
& B R\left(B_{s} \rightarrow D_{s} \pi\right)<15.9 \% \text { at } 95 \% \text { C.L. }
\end{aligned}
$$

and

$$
\begin{aligned}
& f\left(b \rightarrow B_{s}\right) \times B R\left(B_{s} \rightarrow D_{s} \pi\right)<1.6 \% \text { at } 90 \% \text { C.L. } \\
& f\left(b \rightarrow B_{s}\right) \times B R\left(B_{s} \rightarrow D_{s} \pi\right)<2.6 \% \text { at } 95 \% \text { C.L. }
\end{aligned}
$$

This upper limit on the $B_{s}^{0} \rightarrow D_{s}^{-} \pi^{+}$branching ratio relys on $B^{+} \rightarrow \overline{D^{0}} \pi^{+}, \overline{D^{0}} \rightarrow K^{+} \pi^{-}$and $B^{0} \rightarrow D^{-} \pi^{+}, D^{-} \rightarrow K^{+} \pi^{-} \pi^{-}$decays previously unobserved in hadron collider environments. The $B^{+}$and $B^{0}$ signal measured is a $4 \sigma$ effect.

This limit is competitive with the previously published upper limit [1] $]^{10}$

$$
B R\left(B_{s} \rightarrow D_{s} \pi\right)<13.0 \% \text { at } 90 \% \text { C.L. }
$$

based on :

$$
f\left(b \rightarrow B_{s}\right) \times B R\left(B_{s} \rightarrow D_{s} \pi\right)<1.3 \% \text { at } 90 \% \text { C.L. }
$$

but is still an order of magnitude above Standard Model predictions. In addition, ALEPH has recently reconstructed $B_{s}$ mesons via the $B_{s} \rightarrow D_{s} \pi$ channel, but have not cited a branching ratio measurement $[41]$.

\footnotetext{
${ }^{10}$ Assurned $f_{s}=10 \%$
} 


\section{References}

[1] Particle Data Group, Review of Particle Properties, C. Caso et al., E. Phys J. C 3, $1(2000)$

[2] M. Kobayashi, T. Masakawa Prog. Theory Physics, 49, 652 (1973).

[3] S. W. Herb et al., Phys. Rev. Lett. 39, 252 (1977).

[4] F. Abe et al., Phys. Rev. D50, 2966 (1994).

[5] S. Abachi et al., Phys. Rev. Letters 742632 (1995).

[6] T. Toshito [Super-Kamiokande Collaboration], "Super-Kamiokande atmospheric $\nu$ results," hep-ex/0105023.

[7] L. Wolfenstein, Phys. Rev. Lett. 51, 1945 (1983).

[8] N. Cabbibo, Phys. Rev. Lett. 10531 (1963).

[9] B. Aubert et al., hep-ex/0203007 March 2002.

[10] K. Abe et al., hep-ex/0204002 v1 April 2002.

[11] S. Frixione, M.L. Mangano, P. Nason and G. Ridolfi, Heavy Flavours II, eds. A.J. Buras and M. Lindner (World Scientific, Singapore, 1998).

[12] M.L. Mangano, Lectures at the International School of Physics "E. Fermi", Heavy Flavour Physics:A Probe of Nature's Grand Design, CERN-TH/97-328 (1997). 
[13] C. Peterson, D. Schlatter, I. Schmitt and P.MI. Zerwas, "Scaling violations in Inclusive $\mathrm{e}^{+} \mathrm{e}^{-}$Annihilation Spectra" Phys. Rev. D 27, 105 (1983).

[14] B. Andersson, G. Gustafson, G. Ingelman, T. Sjostrand, "Parton Fragmentation and String Dynamics" Phys. Rep. 97, 31 (1983).

[15] S. Gadomski et al., CDF Note 5007 (1999).

[16] N. Cabbibo Phys. Rev. Lett. 10, 531 (1963).

[17] M. Nuebert et al., Heavy Flavours, edited by A.J. Buras and H. Lindner World Scientific, Singapore, (1992).

[18] M.J. Dugan and B. Brinstein Phys. Lett. B 255, 583 (1991).

[19] N. Isgur and M.B. Wise Phys. Lett. B 32, 113 (1989) and Phys. Lett B 37, 527 (1990).

[20] Bortoletto, D. et al., Phys.Rev. D 451 (1992).

[21] Bortoletto, D. et al., Phys.Rev. D 451 (1992).

[22] Albrecht et al., Z Physics C46, (1990).

[23] J. Lee-Franzini et al., Phys Rev. Lett. 65, 2947 (1990).

[24] D. Buskulic et al., Phys.Lett. B 294, 145 (1992).

[25] F. Abe et al., Phys. Rev. Lett. (1993).

[26] F. Abe et al., Phys. Rev. Rev. D 54, 6596 (1996).

[27] B Physics at the Tevatron: Run II and Beyond, FERMILAB-Pub_01/197. 
[28] F. Parodi, P. Roudeau, A. Stocchi, Nuovo Cim. A112 (1992) 833.

[29] G. Garron Stochastic Cooling Test in ICE, Physics Letters B 7, 353,(1978).

[30] H. Wenzel "Tracking in the SVX", CDF Note 1790, (1998).

[31] P. Azzi SVX', the New CDF Silicon Vertex Detector, Nulc. Instrum. Method, A360, 137 (1995).

[32] F. Bedeschi, "Design and Construction of the CDF Central Tracking Chamber", Nucl. Instrum. Meth. A268 50 (1988).

[33] L. Balka, "The CDF Central Electromagnetic Calorimeter", Nucl. Inst. Meth. A267 272 (1988).

[34] S. Bertolucci, "The CDF Central and Endwall Hadronic Calorimeter", Nucl. Inst. Meth. A267 301 (1988).

[35] D. Amidei, "The CDF Trigger" Nucl. Instrum. Method A265 326 (1988).

[36] T. Sjöstrand, Computer Physies Commun. 82, 74, (1994).

[37] J.D. Lewis and P. Avery, "CLEOMC: The CDF interface to the CLEO Monte Carto (QQ)", CDF Note 2724, (1994).

[38] G.J. Feldman and R.D. Cousins, Phys. Rev. D 57, 3872 (1998).

[39] J. Conway, "Setting Limits and Making Discoveries in CDF", CDF Note 5236, (2000).

[40] O. Helene, Nucl. Instrum. Methods Phys.Res. A 212, 319 (1983). 
[41] A. Heister, et al., CERN-EP/2002-016 (2002). 


\section{ProQuest Distribution Agreement}

This Agreement is between the author (Author) and ProQuest LLC, through its ProQuest Dissertation Distribution business (formerly ProQuest/UMI). Under this Agreement, Author grants ProQuest certain rights to preserve, archive and distribute the dissertation or thesis (the Work), abstract, and index terms provided by Author to ProQuest.

\section{Section I. License for Inclusion of the Work in ProQuest Publishing Program}

Grant of Rights. Author hereby grants to ProQuest the non-exclusive, worldwide right to reproduce, distribute, display and transmit the Work (in whole or in part) in such tangible and electronic formats as may be in existence now or developed in the future. Author further grants to ProQuest the right to include the abstract, bibliography and other metadata in the ProQuest Dissertations \& Theses database (PQDT) and in ProQuest Dissertation Abstracts International and any successor or related index and/or finding products or services.

ProQuest Publishing Program - Election and Elements. The rights granted above shall be exercised according to the publishing option selected by Author in Section III, Author Options, and subject to the following additional Publishing Program requirements:

- Distribution of the Work. Except as restricted by Author in the publishing option selected, the rights granted by Author automatically include (1) the right to allow sale and distribution of the Work, in whole or in part, by ProQuest and its sales agents and distributors and (2) the right to make the Abstract, bibliographic data and any meta data associated with the Work available to search engines and harvesters.

- Restrictions. ProQuest will use commercially reasonable efforts to restrict the distribution of the Work as provided under the publishing option selected by Author or as later elected by Author through direct contact with ProQuest. Such election is subject to Author's Degree Granting Institution Directives. With respect to restrictions requested after submission of the Work, Author acknowledges that ProQuest cannot recall or amend previously distributed versions of the Work.

- Removal of Work from the Program. ProQuest may elect not to distribute the Work if it believes that all necessary rights of third parties have not been secured. Refer to the website http://www.proquest.com/products_umi/dissertations/for information about copyright and your dissertation or thesis. If Author's degree is rescinded, and/or the degree-granting institution so directs, ProQuest will expunge the Work from its publishing program in accordance with its then current publishing policies.

- Degree Granting Institution Directives. Author is solely responsible for any conflict between policies and directions of Author's degree-granting institution, Author's choice of publishing model, and/or any restriction Author places on the use of the Work. For the avoidance of doubt, ProQuest is not responsible for access to the Work that is provided by Author's degree-granting institution through its library or institutional repository. Author must work directly with Author's degree granting institution to ensure application of any restrictions to access relating to the Work by Author's degree granting institution.

Delivery of the Work. Author shall provide to ProQuest the Work and all necessary supporting documents during the online submission process, according to the Instructions accompanying this agreement.

Rights Verification. Author represents and warrants that Author is the copyright holder of the Work and has obtained all necessary rights to permit ProQuest to reproduce and distribute third party materials contained in any part of the Work, including all necessary licenses for any non-public, third party software necessary to access, display, and run or print the Work. Author is solely responsible and will indemnify ProQuest for any third party claims related to the Work as submitted for publication.

Open Access Publishing Plus

I want the broadest possible dissemination of my work, and I want to provide free global access to the electronic copy of my work wia the internet.

I understand that I will not be eligible to receive royalties.

I want major search engines (e.g. Google, Yahoo) to discover my work. Learn more: http://www.proquest.com/enuS/products/dissentations/google.shtml

$$
\begin{aligned}
& \square \quad \text { Yes } \\
& \square \quad \text { No }
\end{aligned}
$$

Acknowledgment: I have read, understand and agree to this ProQuest Publishing Agreement, including all rights and restrictions included within the publishing option chosen by measfindicated above.

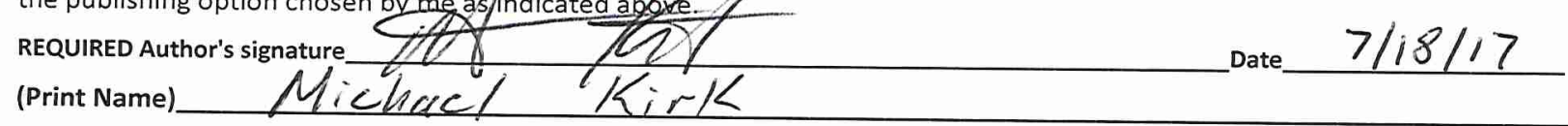

Institution conferring degree Brandeis (Liviversity 


\section{Dissertation/Master's Thesis Submission Form} Please print clearly in block letters

\section{Personal Information}

Last Name $\frac{\text { KirK }}{\text { Mirst Name }}$

\section{Degree \& Dissertation Information}

M (Master's Thesis)

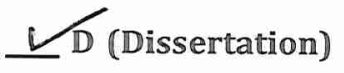

Middle Name or Initial

Country (ies) of Citizenship

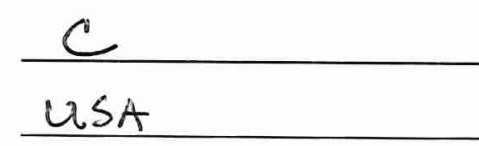
Degree awarded
(abbreviate; e.g., Ph.D.) Ph. D. Year degree awarded 2003 Year manuscript completed
Department or Program

Advisor/Committee Chair

Committee Member

Committee Member

$$
\text { Brandeis University }
$$

Arts \& Sciences.

$$
\text { Physics }
$$$$
\text { Craig Blocker Ph.D. }
$$

Member

Committee

Member 
Primary Subject Category: Enter the 4-digit.sh

$$
\text { code } 0798 \text { category Particle Physics }
$$

You may suggest two additional subject categories that may aid in the discovery of your work in our digital database.

Code

Category

Code

Category

Provide up to 6 keywords or short phrases for citation indices, library cataloging, and database searching.

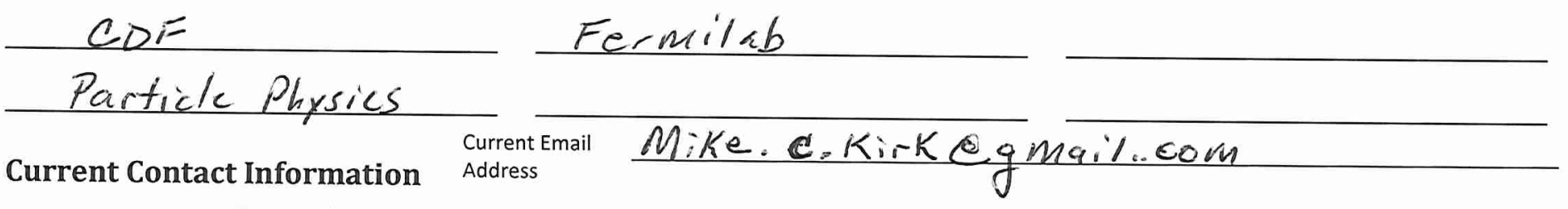

Street Address 34 Vine Si

Please provide your postal address if you are interested in receiving royalties on sales of your thesis.

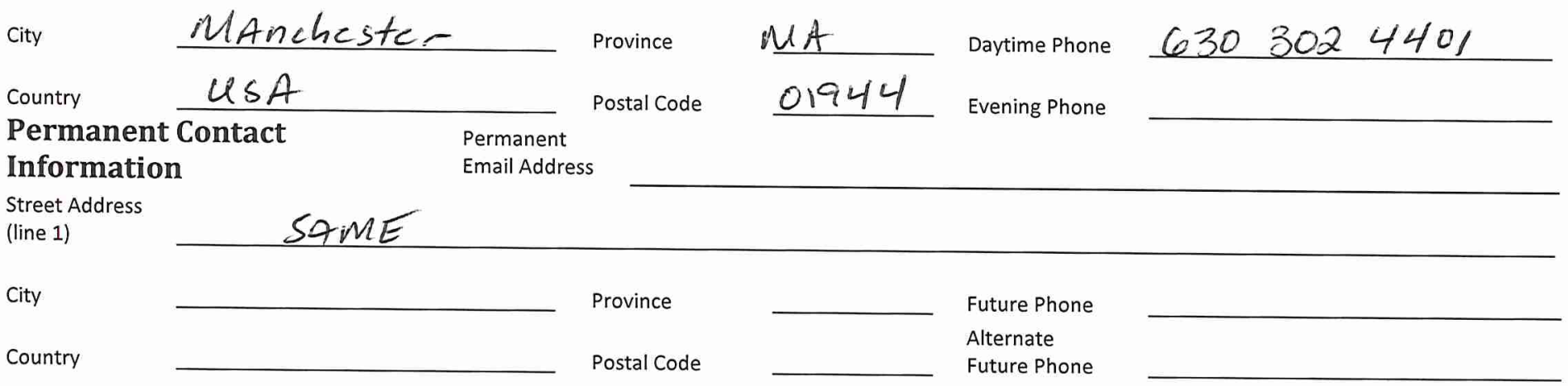

THIS PAGE MUST ACCOMPANY YOUR MANUSCRIPT AND THE REST OF YOUR SUBMISSION MATERIALS

\section{Subject Categories}

The ProQuest Dissertations \& Theses (PQDT) database and the ProQuest citation indices are arranged by subject categories. Please select the one category below that best describes your field of research or creative work. You may add one or two additional categories on your submission form that will also be associated with your work as secondary subjects.

\section{Arts, Business, Education, Humanities, and Social Sciences}

\begin{tabular}{ll}
\multicolumn{2}{l}{ AREA, ETHNIC, AND GENDER } \\
\hline \multicolumn{2}{l}{ STUDIES } \\
\hline African American studies & 0296 \\
African studies & 0293 \\
American studies & 0323 \\
Asian American studies & 0343 \\
Asian studies & 0342 \\
Baltic studies & 0361 \\
Black studies & 0325 \\
Canadian studies & 0385 \\
Caribbean studies & 0432 \\
Classical studies & 0434 \\
East European studies & 0437 \\
Ethnic studies & 0631 \\
European studies & 0440 \\
French Canadian culture & 0482 \\
Gender studies & 0733 \\
GLBT studies & 0492 \\
Hispanic American studies & 0737
\end{tabular}

Holocaust studies

Islamic culture

Judaic studies

Latin American studies

Middle Eastern studies

Native American studies

Near Eastern studies

North African studies

Pacific Rim studies

Regional studies

Scandinavian studies

Slavic studies

South African studies

South Asian studies

Sub Saharan Africa studies

Women's studies
0507

0512

0751

0550

0555

0740

0559

0560

0561

0604

0613

0614

0654

0638

0639

0453

\begin{tabular}{ll} 
BUSINESS & \\
\hline Accounting & 0272 \\
Arts management & 0424 \\
Banking & 0770 \\
Business & 0310 \\
Entrepreneurship & 0429 \\
Finance & 0508 \\
Management & 0454 \\
Marketing & 0338 \\
Sports management & 0430
\end{tabular}

COMMUNICATIONS AND INFORMATION SCIENCES Communication Information science

Library science

Mass communication

Technical communication
0272
0424
0770
0310
0429
0508
0454
0338
0430

0723

0391

0399

0708 0643 
FINE AND PERFORMING ARTS

\section{Art criticism}

0365

Art history

0377

Cinematography

0435

Dance

0378

Design

Film studies

0389

Fine arts

0900

Music

Performing arts

Theater

Theater history

0413

0641

0465

0644

\section{EDUCATION}

Adult education

Art education

0516

Bilingual education

0273

Business education

Community college education

Continuing education 0651

Curriculum development $\quad 0727$

Early childhood education 0518

Education

0515

Education finance $\quad 0277$

Education policy 0458

Educational administration $\quad 0514$
Educational evaluation 0443

Educational leadership $\quad 0449$

Educational psychology 0525

Educational technology $\quad 0710$

Educational tests \& measurements 0288

Elementary education $\quad 0524$

English as a second language 0441

Foreign language instruction $\quad 0444$

Gifted education $\quad 0445$

Health education $\quad 0680$

Higher education $\quad 0745$

Higher education administration $\quad 0446$

History of education 0520

Home economics education $\quad 0278$

Industrial arts education 0521

Instructional design $\quad 0447$

Language arts $\quad 0279$

Mathematics education $\quad 0280$

Middle school education $\quad 0450$

Multicultural education 0455

Music education $\quad 0522$

Pedagogy 0456

Performing arts education $\quad 0457$

Philosophy of education 0998

Physical education 0523

Reading instruction 0535

Religious education $\quad 0527$

School counseling $\quad 0519$

Science education $\quad 0714$

Secondary education 0533

Social sciences education 0534

Sociology of education $\quad 0340$

Special education 0529

Teacher education 0530

Vocational education $\quad 0747$

\section{HUMANITIES}

HISTORY

African history 0331

American history $\quad 0337$

Ancient history 0579

Asian history 0332

Black history 0328

Canadian history 0334

European history 0335

History 0578

History of Oceania $\quad 0504$

History of science $\quad 0585$

Latin American history $\quad 0336$

Medieval history 0581

Middle Eastern history 0333

Military history 0772

Modern history 0582

Russian history $\quad 0724$

World history 0506

LANGUAGE \& LITERATURE

African literature 0316

American literature $\quad 0591$

Ancient languages $\quad 0289$

Asian literature $\quad 0305$

British and Irish literature 0593

Canadian literature 0352

Caribbean literature $\quad 0360$

Classical literature $\quad 0294$

Comparative literature $\quad 0295$

English literature 0593

French Canadian literature $\quad 0355$

Germanic literature 0311

Icelandic \& Scandinavian literature 0362

Language $\quad 0679$

Latin American literature $\quad 0312$

Linguistics $\quad 0290$

Literature 0401

Literature of Oceania $\quad 0356$

Medieval literature $\quad 0297$

Middle Eastern literature $\quad 0315$

Modern language 0291

Modern literature 0298

Rhetoric 0681

Romance literature 0313

Slavic literature $\quad 0314$

PHILOSOPHY AND RELIGION

Aesthetics

Biblical studies 0321

Canon law 0375

Clerical studies $\quad 0319$

Comparative religion 0618

Divinity 0376

Epistemology 0393

Ethics 0394

Logic 0395

Metaphysics 0396

Pastoral counseling 0397

Philosophy 0422

Philosophy of Religion $\quad 0322$

Philosophy of science $\quad 0402$

Religion 0318

Religious history $\quad 0320$

Spirituality 0647

Theology 
LAW AND LEGAL STUDIES

Alternative dispute resolution $\quad 0649$

Intellectual property 0513

International law $\quad 0616$

Law 0398

Patent law $\quad 0562$

\section{SOCIAL SCIENCES}

Archaeology

0324

Area planning and development $\quad 0341$

Criminology

Cultural anthropology $\quad 0326$

Demography 0938

Economic history 0509

Economic theory 0511

Economics 0501

Economics, Commerce-Business 0505

Economics, Labor 0510

Folklore 0358

Forensic anthropology $\quad 0339$

Geography 0366

Individual \& family studies $\quad 0628$

International relations 0601

Labor relations $\quad 0629$

Military studies $\quad 0750$

Organization theory 0635

Organizational behavior $\quad 0703$

Peace studies 0563

Physical anthropology 0327

Political Science 0615

Public administration $\quad 0617$

Public policy 0630

Recreation and tourism $\quad 0814$

Social research 0344

Social structure $\quad 0700$

Social work 0452

Sociolinguistics $\quad 0636$

Sociology 0626

Transportation planning $\quad 0709$

Urban planning 0999
INTERDISCIPLINARY

$\begin{array}{ll}\text { Alternative energy } & 0363 \\ \text { Biographies } & 0304 \\ \text { Climate change } & 0404 \\ \text { Cultural resources management } & 0436 \\ \text { Energy } & 0791 \\ \text { Food science } & 0359 \\ \text { Home economics } & 0386 \\ \text { Information technology } & 0489 \\ \text { Multimedia } & 0558 \\ \text { Museum studies } & 0730 \\ \text { Sustainability } & 0640 \\ \text { Textile research } & 0994 \\ \text { Wood sciences } & 0746\end{array}$

0363

0404

0436

0791

0386

0489

0558

0640

0746

\section{Behavioral, Natural, and Physical Sciences}

\section{AGRICULTURE}

Agriculture

Agronomy

0473

Animal diseases

0285

0476

Animal sciences

0475

Fisheries and aquatic sciences 0792

Forestry

Horticulture

Plant pathology

Plant sciences

Range management

Soil sciences

Urban forestry

Wildlife management

\section{ARCHITECTURE}

Architecture

Architectural engineering

Landscape architecture

0729

0462

0390

BEHAVIORAL SCIENCES

Animal behavior

\section{Behavioral sciences}

Clinical psychology

Cognitive psychology

Counseling psychology

Developmental psychology

Experimental psychology

Occupational psychology

Personality psychology

Physiological psychology

Psychobiology

Psychology

Quantitative psychology and

psychometrics

Social psychology

BIOLOGICAL SCIENCES

Biochemistry

Bioinformatics

Biology

Biomechanics

Biophysics

Biostatistics
Cellular biology

Developmental biology

Endocrinology

Entomology

Evolution \& development

Genetics

Histology

Limnology

Microbiology

Molecular biology

Morphology

Neurosciences

Parasitology

Physiology

Plant biology

Systematic biology

Virology

Zoology
0379

0758

0409

0353

0412

0369

0414

0793

0410

0307

0287

0317

0718

0719

0309

0423

0720

0472 


\section{ECOSYSTEM SCIENCES}

Ecology

Macroecology

0329

0420

Paleoecology

\section{ENGINEERING}

Aerospace engineering 0538

Artificial intelligence $\quad 0800$

Automotive engineering $\quad 0540$

Biomedical engineering 0541

Chemical engineering 0542

Civil engineering 0543

Computer engineering $\quad 0464$

Computer science 0984

Electrical engineering $\quad 0544$

Engineering 0537

Geological engineering 0466

Geophysical engineering $\quad 0467$

Geotechnology 0428

Industrial engineering $\quad 0546$

Mechanical engineering 0548

Mining engineering 0551

Naval engineering 0468

Nanotechnology 0652

Nuclear engineering 0552

Ocean engineering 0547

Operations research 0796

Packaging 0549

Petroleum engineering 0765

Plastics 0795

Robotics 0771

System science $\quad 0790$

ENVIRONMENTAL SCIENCES

Conservation biology

Environmental economics 0438

Environmental education $\quad 0442$

Environmental engineering $\quad 0775$

Environmental geology $\quad 0407$

Environmental health 0470

Environmental justice $\quad 0619$

Environmental law 0439

Environmental management $\quad 0474$

Environmental philosophy $\quad 0392$

Environmental science $\quad 0768$

Environmental studies $\quad 0477$

Land use planning 0536

Natural resource management 0528

Water resources management 0595

Wildlife conservation
GEOSCIENCES

Aeronomy 0367

Atmospheric chemistry 0371

Atmospheric sciences $\quad 0725$

Biogeochemistry 0425

Biological oceanography 0416

Chemical oceanography 0403

Continental dynamics 0406

Geobiology 0483

Geochemistry 0996

Geographic information science
and geodesy
0370

Geology 0372

Geomorphology $\quad 0484$

Geophysics 0373

Hydrologic sciences $\quad 0388$

Marine geology 0556

Meteorology 0557

Mineralogy 0411

Paleoclimate science 0653

Paleontology 0418

Petroleum geology 0583

Petrology 0584

Physical geography 0368

Physical oceanography 0415

Planetology 0590

Plate tectonics 0592

Remote sensing 0799

Sedimentary geology 0594

HEALTH AND MEDICAL SCIENCES

Aging 0493

Alternative medicine $\quad 0496$

Audiology 0300

Dentistry 0567

Epidemiology 0766

Gerontology 0351

Health care management $\quad 0769$

Health sciences $\quad 0566$

Immunology 0982

Kinesiology 0575

Medical ethics 0497

Medical imaging and radiology $\quad 0574$

Medicine 0564

Mental health 0347

Nursing 0569

Nutrition 0570

Obstetrics and gynecology $\quad 0380$

Occupational health 0354

Occupational therapy 0498

Oncology 0992

Ophthalmology 0381

Osteopathic medicine $\quad 0499$

Pathology 0571

Pharmaceutical sciences $\quad 0572$

Pharmacology 0419

Physical therapy 0382

Public health 0573

Public health occupations
education

education

Surgery 0576

Toxicology 0383

Veterinary medicine $\quad 0778$
MATHEMATICAL AND

PHYSICAL SCIENCES

Acoustics

Analytical chemistry

Applied mathematics

Astronomy 
Astrophysics 0596

Atomic physics 0748

Chemistry 0485

Condensed matter physics $\quad 0611$

Electromagnetics 0607

High temperature physics $\quad 0597$

Inorganic chemistry 0488

Low temperature physics $\quad 0598$

Materials science 0794

Mathematics 0405

Mechanics 0346

Molecular chemistry $\quad 0431$

Molecular physics 0609

Nanoscience 0565

Nuclear chemistry 0738

Nuclear physics $\quad 0756$

Optics 0752

Organic chemistry $\quad 0490$

Particle physics 0798

Physical chemistry 0494

Physics 0605

Plasma physics $\quad 0759$

Polymer chemistry 0495

Quantum physics 0599

Statistics 0463

Theoretical mathematics $\quad 0642$

Theoretical physics 0753 